\title{
Genotype by temperature interaction effects on sex determination in zebrafish (Danio rerio)
}

\author{
Dissertation \\ to obtain the $\mathrm{Ph}$. $\mathrm{D}$. degree \\ in the International Ph. D. Program for Agricultural Sciences in Goettingen \\ (IPAG) \\ at the Faculty of Agricultural Sciences, \\ Georg-August-University Göttingen, Germany
}

Presented by

Shahrbanou Hosseini

born in Amol (Iran)

Göttingen, May 2019 
$1^{\text {th }}$ referee: Prof. Dr. Henner Simianer

Animal Breeding and Genetics

Department of Animal Sciences

University of Goettingen

$2^{\text {th }}$ referee: Prof. Dr. Klaus Wimmers

Director of Leibniz Institute for Farm Animal Biology (FBN)

$3^{\text {th }}$ referee: Prof. Dr. Bertram Brenig

Molecular Biology of Livestock and Molecular Diagnostics

Department of Animal Sciences

University of Goettingen

Date of disputation: $\quad 3^{\text {rd }}$ of July, 2019 


\section{TABLE OF CONTENTS}

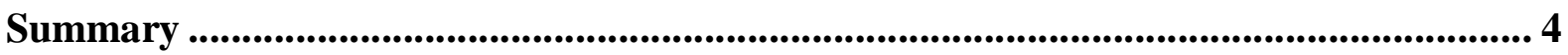

CHAPTER 1: General Introduction ................................................................................ 6

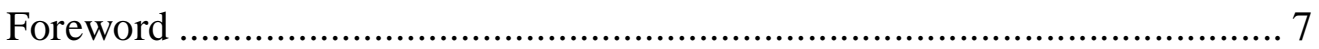

Sexual diversity and plasticity in teleost fish - different means to same end... 7

Main features of sex determination and gonadal dimorphism in zebrafish ... 11

Genetic architecture of sex determination in zebrafish - a polygenic system in

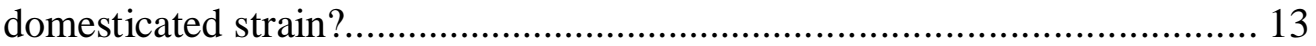

Environmental effects on zebrafish sexual plasticity................................ 15

Sexual selection and mating preference .................................................... 18

Phenotypic plasticity in interaction with environment - does the environment

influence the reliability of the mating signal? ......................................... 21

Objectives of this thesis....................................................................... 23

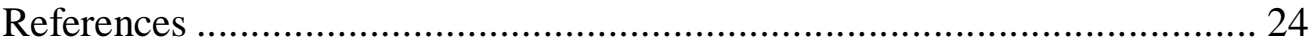

CHAPTER 2: Phenotypic plasticity induced using high ambient temperature

during embryogenesis in domesticated zebrafish, Danio rerio ................. 32

CHAPTER 3: Efficient phenotypic sex classification and assessment of sexual attractiveness in zebrafish using machine learning methods .................. 55

CHAPTER 4: Genetic mechanism underlying sexual plasticity and its association with colour patterning in zebrafish (Danio rerio) .................................. 85

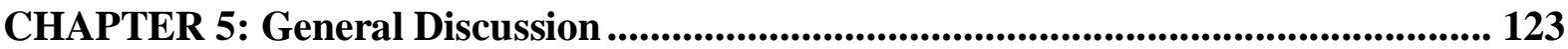

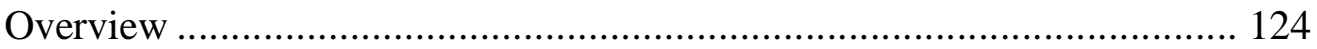

Effects of climate change and phenotypic plasticity............................... 124

Sex determination and quantifying of secondary sexual traits using machine

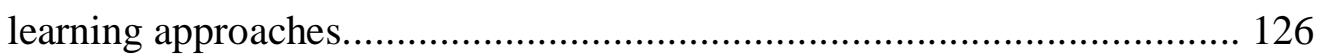

The genetic analysis of sex-associated colour patterns ........................... 128

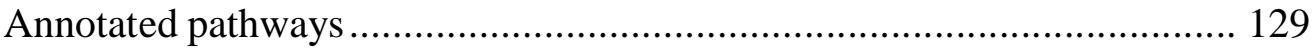

Protein-protein interaction networks.................................................. 133

Specific and shared regulatory mechanisms of sex and colour genes ........ 136

Outlook for future research ............................................................ 139

Main conclusions from this thesis........................................................... 140

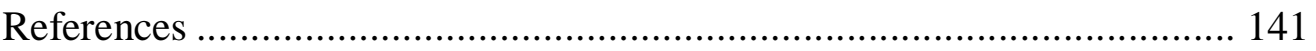




\section{Summary}

The mechanism of sex determination and gonad differentiation in zebrafish is one of the more challenging research questions and complex puzzles in biology. The regulation of sexual dimorphism in this species is not yet fully understood. According to current knowledge, the sex of zebrafish is determined genetically on the basis of a polygenic sex determination system. In this system the interaction of genetic and environmental factors contributes to determine the sexual fate of an organism. The main objective of this thesis was to investigate the effects of high ambient temperature, as one of the major environmental factors, in interaction with the genome on sexual plasticity and phenotypic traits of zebrafish.

Chapter 1 introduces a general overview of sexual diversity in teleost fish and explains the main features of sex determination and gonad differentiation mechanisms in zebrafish. The sexual selection theory in relation to the reproductive success and the effect of environment on secondary sexual traits is generally described.

In chapter 2, the phenotypic plasticity of zebrafish in response to increased water temperature is investigated. The study was designed to test the influence of transient temperature perturbations during the thermosensitive embryonic developmental period (from gastrula to pharyngula) in a high number of families (69 families) on various biological functions. The physiological response to the high ambient temperature revealed a lower hatchability and survival ability during embryonic and post-embryonic development. The results of survival trajectories until adulthood demonstrated that in zebrafish the life stages most sensitive to thermal changes are the first day after fertilization and the first two weeks after hatching. Consideration of the effect of temperature on morphometric traits (weight and length) in order to investigate the temperature $\times$ sex interaction effect indicated a higher growth performance in temperature-treated animals at different time points of adulthood development. A heat-induced masculinization was clearly observed across all families, while a wide range of interfamily sex ratio variations was detected. These observations emphasize a genetic $\times$ environment $(\mathrm{G} \times \mathrm{E})$ interaction of sex determination and constitute a strong confirmation for a polygenic sex determination system in zebrafish.

In chapter 3, a fully automated phenotypic sex classification in zebrafish was established for the first time using two different machine learning methods: Deep Convolutional Neural Networks (DCNNs) and Support Vector Machine (SVM). Based on phenotypic characteristics, a high accuracy of sex differentiation was obtained using these two methods in non-heat-treated groups. However, in treated animals, some males were misclassified using 
SVM due to reduced pigmentation intensity, suggesting these animals were probably masculinized females. Investigation of the colour intensity of the caudal fin using SVM shows that males exhibited a higher pigmentation intensity compared to females. Furthermore, a positive association of male caudal fin colouration with the morphometric traits (weight and length) was determined. These results imply the effect of temperature on secondary phenotypic sexual characteristics, which in turn may influence the sexual attractiveness for mating and reproductive success.

Phenotypic modifications in response to different environmental conditions indicate the changes in the expression of genes responsible for those traits. Hence chapter 4 examines, whether the sex and colour genes are differentially expressed in the two sexes with respect to the observed sexually dimorphic in the caudal fin colouration. This hypothesis is derived from chapter 3, where the sexually differing colour intensity in the caudal fin of the two sexes was detected. Therefore, a transcriptome analysis of caudal fins and gonads was performed in chapter 4 in order to identify the differentially expressed genes and pathways regulating sexual dimorphism in interaction with high water temperature. In addition, the mechanism of gene expression in the masculinization process, as one of the most important consequences of increased temperature, was studied in this chapter. A significantly differentiated expression of sex determination and colour pattern genes was identified in the gonad. In the caudal fin, a high expression magnitude of a set of colour pattern genes was observed, although they were not differentially expressed in two sexes of adult fish. The enrichment of a subset of pathways containing sex and colour genes provides an evidence of the involvement of those genes in the regulation of phenotypic sexual dimorphism in zebrafish. These results led to additional analyses in chapter 5 examining the validity of this hypothesis.

In chapter 5, the topics of previous chapters are first discussed in more detail, and then the hypothesis of chapter 4 is further investigated by pathway analysis, gene interaction networks and transcription factor analysis. All analyses in this context supported the association between sex determination and colour pattern genes in zebrafish, as proposed in chapter 4 of this thesis. The conclusion of this chapter highlights the perspective for future studies answering the open questions derived from this thesis. 


\section{CHAPTER 1}

General Introduction 


\section{Foreword}

Increased water temperatures, predicted as a result of global warming, lead to changes in the complex marine ecosystems and in the ecobiology of fish species resulting in changes in population dynamics, particularly in those with sexual and phenotypic plasticity. In order to gain new insights into the unforeseeable ecological changes, research work is required in order to develop counter-strategies. Zebrafish (Danio rerio), a widely used model animal, shows sexual plasticity and is a suitable organism for studying the effect of environmental factors on phenotypic changes in natural fish populations. The main goal of this thesis is to investigate the influence of elevated water temperature during embryogenesis on sexual and phenotypic plasticity and its underlying genetic mechanism in zebrafish. This chapter gives a general overview of the mechanism of sex determination and phenotypic plasticity in interaction with environmental conditions in zebrafish, followed by a short description of secondary sexual characteristics related to sexual selection and mating preference.

\section{Sexual diversity and plasticity in teleost fish - different means to same end}

This part of the thesis gives a general overview of the complex system of reproductive strategies in teleost fish in order to elucidate the status of zebrafish in this class. The following parts then illustrate its sex determination and gonad differentiation mechanism. In general, sexualities in teleost fish are divided into different categories: gonochorism, hermaphroditism and unisexuality, in which the sex of the gonochoristic species is genetically determined, possess ovaries or testes and retain the same sex throughout their lifespan. In gonochoristic species, the sex of an individual may be directly genetically determined as male or female by the chromosomal composition, or the individual may initially have an indifferent gonad, which subsequently develops into only ovary or testis after sex determination in adulthood. In the latter group, sex may be influenced by environmental factors and determined by a combination of both genetic and environmental factors during early gonadal development, and stay irreversible during their lifetime (Devlin and Nagahama, 2002; Kobayashi et al., 2013; Liu et al., 2017). Gonads in these species are initially developed as ovarian tissue that can transform into the testes in encounters with different environmental conditions during sensitive periods of gonadal development within or beyond the embryonic development, such as Danio rerio and Barbus tetrazona species 
(Uchida et al., 2002; Devlin and Nagahama, 2002). In contrast to the gonochoristic species, the hermaphroditic species possess both male and female gametes at the same time. This mode of sexuality is divided into two groups: synchronous hermaphrodites (also known as simultaneous hermaphrodites) and sequential hermaphrodites. In simultaneous hermaphrodites, some species are able to switch between sperm and egg delivery (outcrossing) and some are even capable to self-fertilization. In sequential hermaphrodites, some individuals are able to produce first one gamete type and then reverse the sex into the other type in a subsequent spawning cycle. This group is classified as protandrous, protogynous and serial bidirectional sex change, in which the sexuality and gonadal phenotype can change from male to female or from female to male or in both directions during adulthood, respectively (Devlin and Nagahama, 2002; Avis and Mank, 2009; Kobayashi et al., 2013; Todd et al., 2016; Liu et al., 2017). Protogyny species can be either monandric fish wherein all males are sex-reversed females in the adulthood, or diandric fish, where the individuals may mature as either males or females from immature bisexual stages (initial phase) and then all sexually matured females can become males in the terminal phase in adulthood (Devlin and Nagahama, 2002; Godwin, 2010). These unique features of sexual patterns in sequential hermaphrodite species have been found in coral reefs and warm water reef fishes. Interestingly, the change of sex in many cases of sequential hermaphroditism is initiated by certain social factors such as disappearance of males or females in a group or population, particularly in bidirectional sex change fishes such as Trimma okinawae (Sakai et al., 2007; Godwin, 2010; Kobayashi et al., 2013). This fish is able to change sex within a few days, where removal of male from a group results in the largest female changing sex to male; when the male returns to the group, the sex-reversed female will switch back to the normal female again (Manabe et al., 2007). Unisexuality is another form of gonadal differentiation in teleost fish that is categorized into three modes of reproduction: gynogenesis, hybridogenesis and parthenogenesis, which produce all female progeny (Heule et al., 2014). In gynogenesis, offspring are formed from maternal genetic information involving either meiotic or mitotic chromosomes, producing all-female progeny in both types. However, in hybridogenesis, interspecific hybrids between closely related species with different sex-factor number and locations in their genome result in intersexuality, sterility, and mostly single-sex progeny (Devlin and Nagahama, 2002). These amazing capabilities of gonadal plasticity and reproductive strategies exist among the vertebrates only in fish (Figure 1). They thus provide an excellent experimental system to study the evolution of sex determination and gonad 
differentiation in many animal species. Furthermore, this diversity implies that the sexual fate in some teleost fish species is not only regulated by inherited genetic factors, but can also be controlled by exogenous factors.

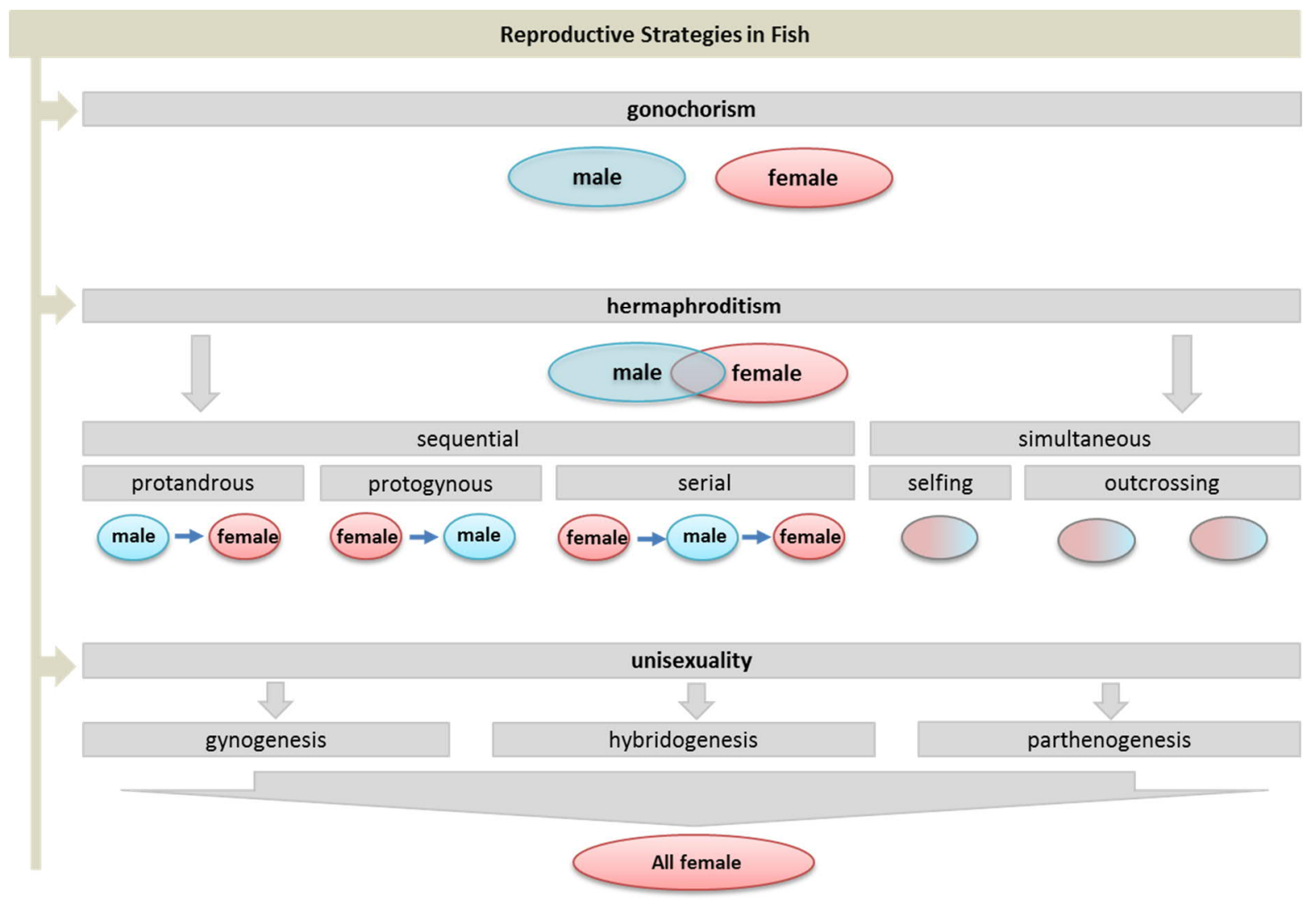

Figure 1: Schematic diagram showing the reproductive strategies in fish species (Modified from Devlin and Nagahama, 2002; Avis and Mank, 2009; Heule et al., 2014).

Sex determination in gonochoristic fish species is divided into two systems: genetic sex determination (GSD) and environmental sex determination (ESD). Temperature-dependent sex determination (TSD) is known to be the most common type of ESD (Figure 2). In the GSD system, where sex is determined by major sex factors, the system of inheritance can be monofactorial - based on the presence of single pair or multiple sex chromosomes - or multifactorial - based on the presence of single pair sex chromosomes. If sex is not inherited by a chromosomal system (minor sex factors), sex is determined by a combination of several factors distributed across the genome (polyfactorial system) (Penman and Piferrer, 2008; Mank and Avise, 2009; Piferrer et al., 2012). In this system, sex can be influenced by environmental conditions and determined by genotype $\times$ environment interactions. A common mode of sex determination in this system is based on genotype $\times$ temperature effect 
$(\mathrm{GSD}+\mathrm{TE})$ interactions, where the temperature can alter the sex of thermosensitive fish species such as European sea bass, Nile tilapia, African catfish, Southern flounder and zebrafish (Ospina-Alvarez and Piferrer, 2008; Piferrer et al., 2012; Shen and Wang, 2014).

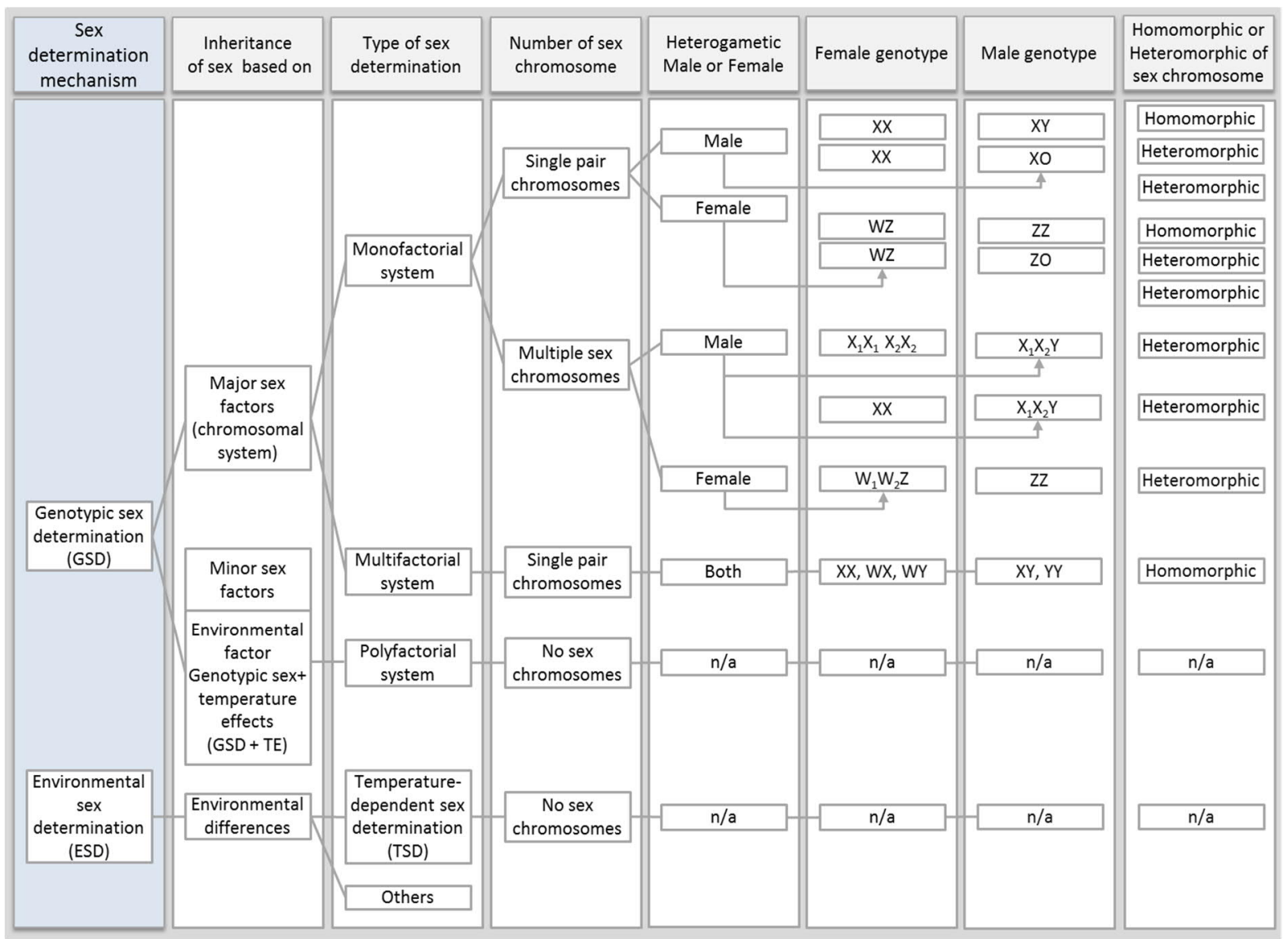

Figure 2: A schematic diagram of sex determination mechanism in gonochoristic fish species. n/a: does not apply (Modified from Devlin and Nagahama, 2002; Penman and Piferrer, 2008; OspinaAlvarez and Piferrer, 2008).

In ESD system, there is no sex determination chromosome and the sex of an individual is determined by various environmental factors such as temperature, $\mathrm{pH}$, photoperiod, density, salinity, and hypoxia (Devlin and Nagahama, 2002; Shang et al., 2006; Penman and Piferrer, 2008). In this system, the environmental factors play a decisive role for the sexual fate of an organism. Studies point to temperature as the most important environmental signal inducing an asymmetric sexual development during thermosensitive developmental periods in some fish species such as cichlid species, which is known as TSD (Valenzuela et al., 2003; OspinaAlvarez and Piferrer, 2008; Luckenbach et al., 2009; Shen and Wang, 2014). 
Hence, teleost fish exhibit a remarkable diversity of sex determination mechanisms, suggesting an instable process of sexual development with respect to evolutionary patterns among taxa. Zebrafish is selected as an ideal model animal for studying sex determination and gonad differentiation in vertebrates. Zebrafish is classified as a gonochoristic species with undifferentiated gonad at the early stage of development, possessing a GSD + TE sex determination system rather than TSD mechanism (Ospina-Alvarez and Piferrer, 2008). However, there is a difference in the genetic makeup of sex determination between wild and domesticated zebrafish strains, which will be explained in more detail in the subsequent parts. In the following, a brief overview is given of the architecture of sex determination and gonadal differentiation in zebrafish in general and in particular of their sex determination mechanism in interaction with ambient temperature.

\section{Main features of sex determination and gonadal dimorphism in zebrafish}

The mechanism of sex determination and gonad differentiation in zebrafish is very complex and the process has not yet been fully clarified. How sex in this species is determined and how the genetic component can control the sex in interaction with environment remains an open question in biology. Studies in the last decades have shown that sex in zebrafish is determined by a combination of germ cells, genetic factors on a polygenic basis and environmental effects during sex determination and gonad differentiation.

In zebrafish, all individuals initially develop an immature ovary-like gonadal tissue (called "juvenile ovary") during early life, regardless of their genetic background for the terminal sex determination. This bipotential gonad can later develop into either mature ovaries or testes. In this process, the number of primordial germ cells (PGCs) in undifferentiated gonads (during embryogenesis) and oocyte apoptosis (during larval stage around 25 days post-fertilization (dpf), Uchida et al., 2002) plays a pivotal role in the sexual fate decision (Siegfried and Nüsslein-Volhard, 2008; Rodríguez-Marí et al., 2010). During embryonic development, the PGCs migrate from a random location in the early embryo (migration happens during 6-24 hours post fertilization (hpf), Richardson and Lehmann, 2010) to the somatic cells of the gonad and merge with these cells in order to form germ cells. A subset of germ cells acquires the ability to operate as germline stem cells with different sex-specific functions and later differentiate into the gametes (Raz, 2003; Richardson and Lehmann, 2010; Liu et al., 2015). In this step, loss or decrease in the number of germ cells leads to the transition of the primordial gonad from the ovary-to-testis at the final gonadal differentiation process and 
development into a mature testis (Uchida et al., 2002; Hsiao and Tsai, 2003; Wang et al., 2007, Liu et al., 2015; Luzio et al., 2016). It is assumed that the reduction in the number of germ cells could possibly alter the hormonal balance through the steroidogenic pathway and the developmental trajectory tends towards the testicular fate (Dranow et al., 2013). A further assumption is that a germ line deficit occurs in the absence or in the lower threshold number of the germ cells. The germ line affects the sexual fate of the surrounding somatic gonadal tissue, in which its deficiency leads to the initiation of testis specification in the developing gonad and subsequent development of a male (Siegfried and Nüsslein-Volhard, 2008). In addition, oocyte apoptosis in the larval stage also leads to ovary to testis transformation. This mechanism is mainly regulated by expression of key testicular differentiation genes and inhibition of ovarian aromatase genes leading to testicular development.

In contrast to development of testis, maintenance of PGCs during embryonic development, which later form germ cells in order to produce gametes, and inhibition of oocyte apoptosis in the larval stage leads to development of ovaries. One of the assumptions for this process is that the PGCs may regulate the expression of the sex determination genes through a signal derived from the surrounding somatic cells of the gonad to promote the ovary formation and inhibit testicular differentiation (Siegfried and Nüsslein-Volhard, 2008). Furthermore, since the germ cells, as mentioned above, play a leading role in different stages of oocyte development such as oogenesis, ovarian differentiation and oocyte maturation in female gonad (Liu et al., 2015), it has been hypothesized that the germ cells send a signal to the bipotential somatic gonad to induce a female sexual fate. This signal may induce prefollicular cells to adopt the fate of granulosa cells instead of sertoli cells in the juvenile ovary stage to develop the ovarian tissue in the terminal sex determination process (Dai et al., 2015; Nagabhushana and Mishra, 2016). In accordance with these hypotheses, recent observations indicate that the presence of germ cells is necessary even in adult fish in order to preserve the fate of the female gonad, where an individual without a germ line or germ cells developed into a male (Siegfried and Nüsslein-Volhard, 2008; Dranow et al., 2013). In this regard, further research on developing gonads in zebrafish have identified some sex-determining genes involved in different signalling pathways playing an important role in the regulation of the gonadal fate and sex determination, which are explained in the following part about genetics. Further information about the germ cells regulation of sex determination during embryogenesis in zebrafish and its interaction with ambient temperature is given in chapter 2. 


\section{Genetic architecture of sex determination in zebrafish - a polygenic system in domesticated strain?}

In regard to the genetic aspect of sex determination, the question arises how sex is regulated in zebrafish and which genes are involved in this process. As described above, zebrafish has a GSD system that can interact with environmental factors. Several studies have been conducted in recent years to understand the potential role of the genetic component of sex determination in zebrafish. Surprisingly, most of them did not recognize the heteromorphic sex chromosomes (Pijnacker and Ferwerda, 1995; Daga et al., 1996; Gornung et al., 1997; Amores and Postlethwait, 1999; Traut and Winking, 2001; Bradley et al., 2011; as reviewed by Nagabhushana and Mishra, 2016; Santos et al., 2017). In this regard, a recent study of a large number of families in different zebrafish strains showed a wide range of sex ratio among different families, indicating sex as a complex trait influenced by multiple genes (Liew et al., 2012). This suggests that zebrafish has a polygenic sex determination (PSD) system (Liew et al., 2012; Liew and Orban, 2014). In this system, sex is determined by a combination of several alleles of sex-determining genes that are distributed across the genome. In this context, further attempts have been made to identify the variations of these alleles using genome-wide analysis (Bradley et al., 2011; Anderson et al., 2012; Howe et al., 2013; Wilson et al., 2014). The study of genome-wide linkage analysis by Bradley et al. (2011) demonstrated two sex-linked loci on chromosome 5, containing fancg and dmrt1 genes, and on chromosome 16 comprising the cyp21a2 gene. Similarly, a sex-linked locus on chromosome 16 was observed via sequencing the zebrafish genome (Howe et al., 2013). However, the identified locus on chromosome 16 in the study of Howe et al. (2013) did not overlap with the previous study. Another genome-wide linkage study using sequence-based polymorphic restriction-site associated DNA (RAD-tag) markers has shown two sex-linked regions on chromosome 3 and 4 . The result of the later study showed that the sex linked regions contained a few genes such as $h s d 17 b 1$ on chromosome 3 , which may play a role in sex determination of zebrafish, and miRNA (mir430) on chromosome 4, which has a function in PGCs regulation and migration (Anderson et al., 2012; Nagabhushana and Mishra, 2016).

Despite the results of these studies, the current evidence for sex-associated regions in zebrafish reveals that they differ surprisingly among wild and domesticated strains (Wilson et al., 2014). The sex-linked polymorphisms using RAD-tags mapping in the study of Wilson et al. (2014) in two domesticated, two natural laboratory and two wild zebrafish strains have 
shown a sex-linked locus in the right telomere of chromosome 4 in all natural strains. However, it was not observed in the domesticated strains. This finding suggests that there is a chromosomal sex determination system (female-heterogametic: ZW/ZZ) in wild populations, while the domesticated strains have a lack of sex-linked loci. However, a sex-linked region on chromosome 4 as identified in wild populations by Wilson et al. (2014) lacks any known candidate sex-determining genes as aforementioned (Anderson et al., 2012). This result leads to a hypothesis that the domesticated strains may lose the sex chromosome during the domestication process (Wilson et al., 2014; Santos et al., 2017). Furthermore, this result also supports the PSD in domesticated zebrafish strains in accordance with the previous studies (Liew et al., 2012; Liew and Orban, 2014).

In addition, several studies were carried out investigating the molecular mechanism of sex determination and gonad differentiation in zebrafish in order to identify sex genes and gonadal differentiation pathways. In these studies, several pro-male and pro-female genes are reported (refer to the review articles by Nagabhushana and Mishra, 2016; Santos et al., 2017). In the following the most important sex determination genes in zebrafish are introduced, which play a pivotal role in male and female gonad development pathways. The full list of candidate sex genes is explained in chapter 4. Dmrtl is identified as one of the most important sex determination genes, which along with amh and sox9 plays a key role in developing male gonad (Chiang et al., 2001; Guo et al., 2005; Siegfried, 2010). A recent molecular study confirms the role of $d m r t l$ in male zebrafish gonadal sex differentiation, and supports the earlier report of dmrtl by Bradley et al (2011), which was observed in a sexlinked locus on chromosome 5. They found that this gene is located upstream of amh and downstream of sox9a in the testis differentiation pathway and its expression is necessary for activation of the transcriptional regulation of $a m h$ in sertoli cells (Webster et al., 2017). Another important sex determination gene in zebrafish is an aromatase gene (cyp19ala), which plays an important role in ovary development. This gene encodes the aromatase enzyme that catalyzes the conversion of androgens to oestrogens in ovarian differentiation pathways (Rodríguez-Marí et al., 2005). FoxL2, a forkhead transcription factor, is a component of the vertebrate ovary differentiation pathway (Nagabhushana and Mishra, 2016). This gene was first identified in goats, where the deletion of its chromosomal region leads to the polled intersex phenotype that is characterized by loss of horns and XX sex reversal progeny (Pailhoux et al., 2001; Siegfried, 2010). In mammals, foxL2 is co-expressed with aromatase gene in female sex determination pathways and promotes the expression of 
cyp19ala for ovarian development. In zebrafish, this gene is initially expressed in the juvenile ovary stage and its expression later is restricted only in ovaries, which in turn may play a role in ovary development, similar to mammals (Siegfried, 2010). In this regard, a recent study confirmed both cyp19ala and foxL2 as pro-female genes in zebrafish ovary development (Ribas et al., 2017a). Furthermore, several other genes including wnt4, $R$ spondinl and beta-catenin are also likely to play a role in female sex determination pathways (Siegfried, 2010). During zebrafish gonad differentiation, the actions of several signalling pathways regulate the gonadal fate in the juvenile ovary stage to develop a testis or an ovary (Santos et al., 2017). The important known pathways in this process are Piwi/piRNA (Houwing et al., 2007), Tp53/fancl (Rodríguez-Marí et al., 2010), brca2 (Rodríguez-Marí et al., 2011), apoptosis (Rodríguez-Marí et al., 2010 and 2011), nuclear factor kappa B (NF-кB) (Pradhan et al., 2012), and canonical Wnt (Sreenivasan et al., 2014) pathways. Following the juvenile ovary phase, the gonad may continue to develop an ovary by upregulation of female developmental pathways or it undergoes a transformation process to form a testis by upregulation of male developmental pathways. Despite the genetic factors that regulate the gonad differentiation, this process can interact with different environmental factors, which is explained in the following section.

Identification of multiple sex-associated loci on different chromosomes in various zebrafish strains and lack of sex chromosomes suggest that sex in zebrafish is a complicated trait. In this regard, a number of candidate sex determination genes in zebrafish using RNAsequencing (RNA-Seq) approaches are investigated in chapter 4 of this thesis in order to gain new insights into the complex gene regulatory networks of sex determination in this species in interaction with ambient temperature.

\section{Environmental effects on zebrafish sexual plasticity}

Various environmental factors such as hypoxia (Shang et al., 2006; Lo et al., 2011), density (Ribas et al., 2017b), nutrition (Lawrence et al., 2008), temperature (Uchida et al., 2004; Abozaid et al., 2011; Brown et al., 2015; Ribas et al., 2017a) and hormonal stress (Lee et al., 2017) have been reported in several studies as influencing sex determination in zebrafish. However, zebrafish does not have an ESD system like some turtles and fish species, where the environmental signals act as a decisive factor for sex determination (Ospina-Alvarez and Piferrer, 2008). Sex in zebrafish is determined by interplay between genetic and environmental factors during critical period of sex determination and gonad differentiation. 
Most of the studies indicat that environmental factors lead to an increase in the proportion of males, which is possibly due to downregulation of several genes involved in the ovary developmental pathways and shifting the hormonal balance toward maleness (Nagabhushana and Mishra, 2016; Santos et al., 2017). Of these, temperature is one of the most common environmental factors that have influenced the sex ratio of at least 61 documented fish species (Baroiller et al., 2009). In zebrafish, several studies indicated that an increase in water temperature during gonad transformation leads to masculinization. It has been proposed that high water temperature inhibits the expression of aromatase genes affecting the activity of estrogens enzymes involved in the synthesis of sex steroids (Uchida et al., 2004; Wang et al., 2007). The influence of elevated water temperature on sex determination of zebrafish in the PSD system with respect to masculinization deals with two different events in embryonic and larval stages. In the first scenario, it is assumed that a high water temperature during embryonic development may induce degeneration of PGCs (Baroiller et al., 2009; Abozaid et al., 2011), which seems essential for the formation of the initial ovary and the maintenance of female gonads (Siegfried and Nüsslein-Vollhardt, 2008). In the second scenario, it is supposed that the increase in water temperature at the larval stage during gonad differentiation periods leads to apoptosis of oocytes in the juvenile ovary by inhibiting the expression of the aromatase gene and activating the testicular developmental gene (Uchida et al., 2004; Ribas et al., 2017a). Both scenarios lead to sex reversal of genotypic females to phenotypic males (masculinization) in zebrafish. The function of three major gonadal developmental pathways of the above mentioned signalling pathways for gonad development in this process is explained below. These pathways have been shown to be involved in the sexual fate decision of zebrafish through the modulation of programmed cell death (Liew and Orban, 2014), in which their activation can be influenced by environmental factors. In the juvenile ovary stage, upregulation of tp53 signalling pathway (a pro-male pathway) induces activation of apoptotic processes in the gonads resulting in degeneration of oocytes and the hormonal balance shifting toward maleness, and thereby testicular differentiation. In contrast, downregulation of pro-male pathway leads to upregulation of NF- $\mathrm{BB}$ and Wnt signalling pathways (pro-female pathways), which suppress oocyte apoptosis and the hormonal balance is shifted toward femaleness (Figure 4) (Rodríguez-Marí et al., 2010; Pradhan et al., 2012; Liew and Orban., 2014). Other pathways may also participate in zebrafish sex differentiation and gonad transformation, as shown in the previous section. In addition, in this process the number of PGCs has a pronounced effect on oocytes development and the maintenance of the 
ovaries (Liew and Orban, 2014). Hence, high ambient temperature can lead to masculinization by upregulation of pro-male pathway and downregulation of pro-female pathway resulting in activation of apoptosis process and testicular development. This process leads to an increase in the proportion of males and thus generates a male-biased sex ratio in zebrafish population (Abozaid et al., 2011; Liew and Orban, 2014; Ribas et al., 2017a).
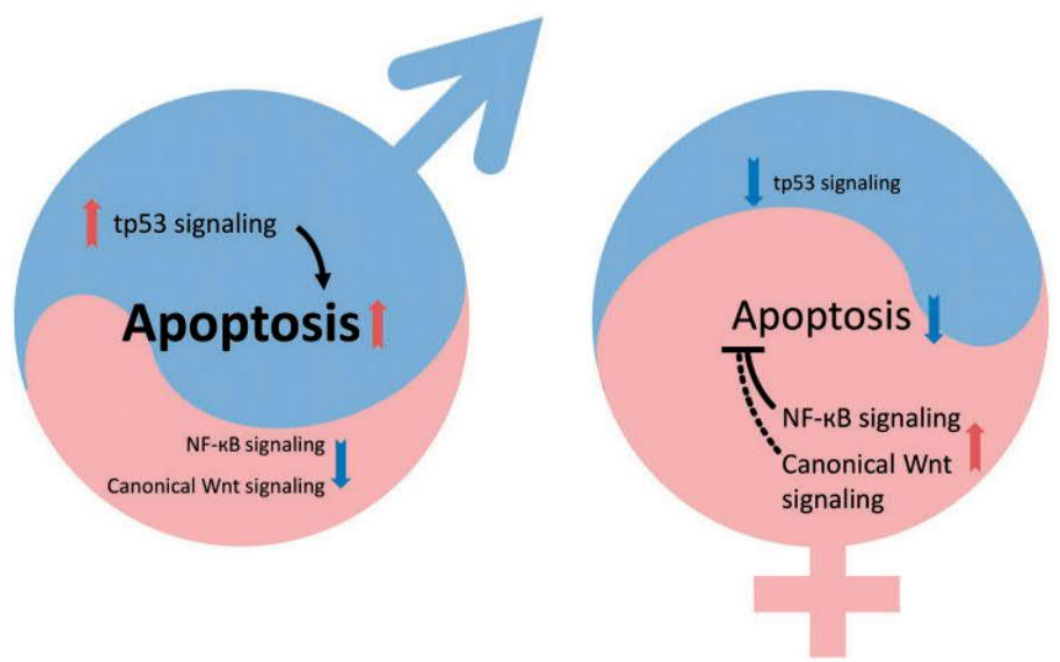

Figure 4: This figure illustrates the activation of major developmental pathways in male gonad (left side) and in female gonad (right side) that conduct the development toward one of the two gonad types. This leads to the shift in the balance of pro-male and pro-female pathways to determine the gonadal fate in zebrafish. Upregulation of tp53 pathway leads to the activation of apoptosis process and downregulation of Wnt signalling and NF- $\mathrm{BB}$ resulted in testis development (left side). In contrast, downregulation of tp53 pathway leads to the repression of apoptosis activation and upregulation of Wnt signalling and NF- $\mathrm{KB}$ resulted in ovary development (right side). (From Liew and Orban, 2014)

This shows that sex in zebrafish is highly plastic and zebrafish has a noteworthy ability to modify their phenotype in dependence of environmental factors. Notwithstanding numerous efforts to understand the mechanisms underlying temperature-induced masculinization in zebrafish, there is still little information on the molecular basis of this process and its effects on sex ratio, particularly during embryogenesis (Abozaid et al., 2011). Thus, thermosensitive fish species may not be able to adapt rapidly to the changes in temperature during global warming, so that temperature fluctuations will have an impact on population dynamics (Shen and Wang, 2014). Sex ratio is the most important demographic parameter influencing the 
structure of fish populations regardless of the mode of reproduction (Penman and Piferrer, 2008). Considering the current climate changing scenarios (IPCC, 2013), further research is necessary to gain more knowledge into the level of temperature that interferes with sexual development of zebrafish, as a model animal, not only to understand its biology but also to conclude the potential implications of global warming on fish populations in the natural ecosystem (Santos et al., 2017). In this regard and based on the mechanism of sex determination in zebrafish and its interaction with environmental factors, we studied the influence of high ambient temperature on phenotypic plasticity such as sex ratio, mortality and morphometric traits in zebrafish as described in chapters $\mathbf{2}$ and $\mathbf{3}$ of this thesis.

\section{Sexual selection and mating preference}

Sexual selection is the success of some individuals over others of the same sex who are selected by opposite sex as mating partners for reproduction. However, natural selection is the success of both sexes in general conditions of their life. Hence, sexual selection is a mode of natural selection, in which some members of one biological sex who have a better reproductive success than others are more attractive partners for mating and producing offspring. Charles Darwin proposed the first conception of sexual selection theory 150 years ago in his book The Descent of Man (1871). In this book, he described two agents for sexual selection: "weapons" and "ornaments" that are found in males of many animal species who try to demonstrate their fitness to be chosen by females for mating (Darwin, 1871; CluttonBrock, 2007; Jones and Ratterman, 2009; Hunt et al., 2009). The traits developed for sexual attractiveness termed as secondary sexual characteristics that influence the reproductive success. These traits lead to sexual dimorphism such as ornate plumage of birds, antlers of deer and manes of lions. Darwin explained intensive reproductive competitions between males to gain breeding opportunities (termed as intrasexual selection), however it is the females who generally choose their breeding partners (termed as intersexual selection) (Clutton-Brock, 2017). Researchers began to investigate Darwin's theory nearly seven decades after his observations. Bateman (1948), an English geneticist of fruit flies (Drosophila melanogaster), was inspired by Darwin's sex role concept and demonstrated that mating and reproductive success varies more widely in male fruit flies than in females. Bateman discovered that the increase in reproductive success rises sharply with the increase in the number of matings in males compared to females (Bateman, 1948; Janicke et al., 2016; Clutton-Brock, 2017). He suggested that females play a greater role in reproduction than 
males, since males are able to produce a million numbers of sperm with little effort, while females invest a higher level of energy to produce a small number of eggs. Generally, females have a larger investment in producing offspring than males. Indeed, males can easily increase their reproductive success by mating with multiple partners, whereas females will not able to produce more offspring by mating with more than one male. Thus, females can fertilize all eggs from a single mating and they do not have to compete for access to mates. Thus, a male's potential reproductive success depends on the number of females that he mates with, whereas a female's potential reproductive success is limited by the number of produced eggs. Therefore, according to the Bateman's principles, males compete with each other in order to be selected as a mating partner and copulate successfully with females, while females choose males with whom they wish to mate (Bateman, 1948; Clutton-Brock, 2017; Hare and Simmons, 2019). Bateman's concept is in accordance with Darwin's sexual selection theory. The combination of Bateman's principles with Darwin's conception known as the "Darwin-Bateman paradigm", became the most common concept of sexual selection for exploring the conventional sex roles across the animal kingdom (Dewsbury, 2005; Janicke et al., 2016). Bateman's principles provide conceptions for understanding of two major sex roles: female-biased parental care and male-biased sexual dimorphism. These two points were developed by Trivers (1972) in the concept of parental investment. Trivers argued that reproductive competition is more intensive in the sex having a lower parental investment to produce offspring (typically males) than in the sex having a higher parental investment (typically females), because there are more individuals competing for breeding opportunities at the same time in the group of lower parental investments (Trivers, 1972; Clutton-Brock, 2017). He proposed that the sex with lower investments has intrasexual competition for mating success and the sex with greater investment in offspring will choose their mating partner. This theory explains sex differences and mate preferences in relation to the theory of sexual selection.

Despite the well-founded sexual selection theory, some researchers proposed that sexual selection theory is a mistake or flawed theory in biology. They argued that Darwin had forgotten some other aspects of sexual selection such as social selection or social competition for mating success (Roughgarden et al., 2006), when other factors dominate in the interaction between males and females for reproductive success such as female choice (Eberhard, 2009; West-Eberhard, 2014). In this regard, Roughgarden et al. (2006) proposed that the Darwinian theory of sexual selection is wrong and needs to be replaced by a novel theory of social 
selection. They believed that both sexes cooperate and equally invest to increase the number of their offspring. Their theory explained the reproductive social behavior aspect with cooperative game theory to maximize the production of offspring. However, the sexual selection theory proposed that females select their mating partners who have the genetically highest quality for reproduction. Therefore, Roughgarden et al. (2006) rejected the female choice theory of Darwin's concept. In this context, many of evolutionary biologists disagreed with the social selection theory and it was rejected by this community in the same year (Pizzari et al., 2006). Some other studies also reported the contradictions of the DarwinBateman paradigm in reproductive competition, parental investment, and sexual differences in behavior and morphology (Gowaty and Hubbel, 2005; Tang-Martinez and Ryder, 2005; Kokko et al., 2006; Clutton-Brock, 2007). They believed that Darwin paid little attention to sexual selection in females, since secondary sexual characteristics were observed in females of some species (Roughgarden et al., 2006; Clutton-Brock, 2007). Some researchers even proposed a new hypothesis of a "gender-neutral" model, in which sex is driven stochastically or by some ecological, demographic and social conditions (Hubbell and Johnson, 1987; Gowaty and Hubbel, 2009; Roughgarden, 2015; Janicke et al., 2016). The criticism of this theory is that it is not able to explain the consistent sex differences in sexual selection as proposed by Darwin-Bateman paradigm (Parker and Birkhead, 2013; Janicke et al., 2016). Hence, Darwin's arguments for sexual selection are still controversial in evolutionary biology and needs further research. However, the observations of female preference for male ornaments in some fish, birds, reptiles and mammals strongly support his theory (Majerus, 1986; Andersson and Simmons, 2006; Janicke et al., 2016). This theory is now one of the most important concepts for reproductive strategies among taxa in evolutionary biology. In this regard and based on the Darwin-Bateman paradigm, a recent study using meta-analysis by standard Bateman metrics (the opportunity for selection, the opportunity for sexual selection and the Bateman gradient) in 72 studies on 66 animal species confirmed Darwinian sex roles across the animal kingdom (Janicke et al., 2016). They found that sexual selection is stronger in males than in females for polygamous species and rejected the stochastic sex roles in the animal kingdom. The sexual selection by ornamentation in zebrafish has not yet been investigated. Therefore, the sexual dimorphism in zebrafish using differences in ornamentation is studied in chapter 3 of this thesis. 


\section{Phenotypic plasticity in interaction with environment - does the environment influence the reliability of the mating signal?}

Phenotypic plasticity describes a change in an organism including behavior, morphology and physiology in response to environmental conditions. Since human activities are rapidly changing the environment, more attention has recently been paid to population responses to environmental perturbations and to predicting their future impact on ecosystems, particularly for endangered species. Environmental changes could disrupt the link between individual quality and sexual signalling trait expression, especially in individuals with genotypeenvironment interactions. In fact, these individuals, who have a different genotype, respond differently to the environmental changes and alter the expression of sexually selected traits (Greenfield and Rodriguez, 2004; Kokko and Heubel, 2008; Candolin and Heuschele, 2008). Evidence shows the influence of the environment on phenotypic plasticity and its interaction with the genotype on sexual traits, which may lead to dishonest signals for mating choice (Qvarnstrom, 1999; Jia et al., 2000; Candolin and Heuschele, 2008). Sexually signalling traits at the individual level are often costly and their benefits can be influenced by environmental conditions (Emlen and Oring, 1977; Candolin and Heuschele, 2008). For example, the darkmaned males in the African lion, Panthera leo, have higher sexual attractiveness and mating success. However, the increase in body temperature caused by dark colour leading to reduced sperm quality and low food intake. It is therefore predicted that an elevated temperature during global warming will lead to an increase in the cost of dark manes to produce offspring (West and Packer, 2002). Hence, environmental changes influence the individual's fitness in terms of the number or quality of offspring, which in turn could influence on population dynamics (Candolin and Heuschele, 2008).

Colour patterning in animals plays an important role for sexual attractiveness and is known as an honest signal from an individual for mating (Reudink et al., 2015). Sexual behaviour and body colour patterns and their associations as complex traits have an important influence on the fitness of an individual. In an evolutionary biology aspect, natural selection in one trait may directly or indirectly influence other traits, which are known as correlated traits (Horth, 2003). For instance, the colouration and aggression in mosquitofishes, a relative of the guppy, are correlated traits in temperature-sensitive melanic males (Martin, 1977; Horth, 2003). The effect of ambient temperatures in some populations of mosquitofishes (temperature-sensitive melanic genotype) at different seasonal temperatures shows the relation between colouration 
and aggression. In this species, black body colouration is expressed under cold temperature condition (late summer), while a silver body colour pattern is present in high ambient temperature (early summer). The melanic males are more aggressive towards females for mating, chasing and high sexual activity than silver males. The survivability of melanic males at higher frequency situation is lower due to mating competition compared to the lower frequency. However, they have a higher survivability in the presence of predators than silver males and may have a mating benefit due to aggression, resulting in a selective advantage for the survival of this genotype, which has a low frequency in nature (Horth, 2003). Another study investigated the effect of environmental conditions and colour polymorphism on sexual selection in Telmatherina sarasinorum fish. In this species, the conspicuous males (blue and yellow colours) have a high reproductive fitness compared to other male morphs in different environment (shallow beach sites and root sites drop off steeply from the shore) (Gray et al., 2008).

In zebrafish, females show a mating preference for larger males - male body size is a sexual selection signal (Pyron, 2003; Skinner and Watt, 2007; Uusi-Heikkilä et al., 2012). However, male zebrafish also show a more intense yellow colouration than females, particularly upon sexual activity (Singh and Nüsslein-Volhard, 2015), which is thought to be important for sexual attractiveness and reproductive success. Furthermore, a recent study indicated a loss of pigmentation in zebrafish exposed to high ambient temperatures during larval stage (Ribas et al., 2017a). This leads to the following questions: first, whether the ambient temperature during embryogenesis can influence the colouration as a mating signal and, second, if there is an association between colour intensity and body size in zebrafish, and if there is how strong it is. We have addressed and discussed these questions in detail in the chapter $\mathbf{3}$ of this thesis. In addition, since the regulation of sex-biased gene expression plays a major role in phenotypic dimorphism (Small et al., 2009) and the expression of sex determination genes might be associated with colour pattern genes in terms of sexual attraction (Sharma et al., 2014), in a further step of this research work the underlying genetic mechanisms of sex determination and colour pattern genes and their interactions in respect to the sexual dimorphism were studied in chapter 4 . 


\section{Objectives of this thesis}

The physiological response to the environmental changes in aquatic ecosystems can increase the risk of extinction and may affect the biodiversity of fish populations. Climate change, as a major environmental factor, has a severe impact on wild-type fish populations due to their lower adaptive capabilities in response to environmental changes, particularly in populations living in shallow waters such as rivers, lakes and coastal areas. Masculinization is one of the most important physiological responses to increased water temperature in fish species with sexual plasticity. The overall objective of this thesis was to study the genotype $\times$ temperature interaction effects on sexual plasticity and phenotypic traits induced by thermal stress during embryogenesis in order to gain new insights into the mechanisms of genetic regulation for sex determination and colour pattern formation in zebrafish as a model animal. The purpose of the subsequent chapters of this thesis is:

Chapter 2: In this chapter, we investigate the effect of high ambient temperature during embryogenesis on sex ratio imbalances and survival trajectories during embryonic and postembryonic developmental stages. We analyze the impact of temperature treatment on morphometric traits of adult zebrafish at different developmental time points.

Chapter 3: We use two fully automatic machine learning methods as procedures for sex classification in zebrafish: Deep Convolutional Neural Networks (DCNNs) based on the whole fish body image and Support Vector Machine (SVM) using the colour distribution of the caudal fin pictures. Then, the estimated colour intensity based on SVM is used to detect the association between colouration and morphometric traits (weight and length), in regard to sexual attractiveness.

Chapter 4: In this chapter, we study the underlying molecular mechanisms of sex determination and colour pattern genes and their interactions in response to high ambient temperature on their expression in the gonads and caudal fins using transcriptome analysis and discuss the association between these two groups of genes.

Chapter 5: We discuss a general effect of increasing water temperature on phenotypic plasticity and secondary sexual characteristics. In this chapter, we further investigate the association between sex determination and colour pattern genes in zebrafish. 


\section{References}

Abozaid H, Wessels S, Hörstgen-Schwark G. Effect of rearing temperatures during embryonic development on the phenotypic sex in zebrafish (Danio rerio). Sex Dev. 2011; 5:259-265.

Amores A, Postlethwait JH. Banded chromosomes and the zebrafish karyotype. Methods Cell Biol. 1999; 60:323-338.

Anderson J1, Rodríguez Marí A, Braasch I, Amores A, Hohenlohe P, Batzel P, Postlethwait JH. Multiple sex-associated regions and a putative sex chromosome in zebrafish revealed by RAD mapping and population genomics. PLoS One. 2012; 7:e40701.

Andersson M, Simmons LW. Sexual selection and mate choice. Trends Ecol Evol. 2006; 21:296-302.

Avise JC, Mank JE. Evolutionary perspectives on hermaphroditism in fishes. Sex Dev. 2009; 3:152-63.

Baroiller JF, D'Cotta H, Saillant E. Environmental effects on fish sex determination and differentiation. Sex Dev. 2009; 3:118-35.

Bateman AJ. Intra-sexual selection in Drosophila. Heredity. 1948; 2:349-368.

Bradley KM, Breyer JP, Melville DB, Broman KW, Knapik EW, Smith JR. An SNP-Based Linkage Map for Zebrafish Reveals Sex Determination Loci. G3. 2011; 1:3-9.

Brown AR, Owen SF, Peters J, Zhang Y, Soffker M, Paull GC, Hosken DJ, Wahab MA, Tyler CR. Climate change and pollution speed declines in zebrafish populations. Proc. Natl. Acad. Sci. U. S. A. 2015; 112:E1237-1246.

Candolin U, Heuschele J. Is sexual selection beneficial during adaptation to environmental change?. Trends Ecol Evol. 2008; 23:446-52.

Chiang EF-L, Pai C-I, Wyatt M, Yan Y-L, Postlethwait J, Chung B. Two sox9 genes on duplicated zebrafish chromosomes: expression of similar transcription activators in distinct sites. Dev Biol. 2001; 231:149-163.

Clutton-Brock T. Reproductive competition and sexual selection. Philos Trans R Soc Lond B Biol Sci. 2017; 372:20160310.

Clutton-Brock T. Sexual Selection in Males and Females. Science. 2007; 318:1882-5.

Daga RR, Thode G, Amores A. Chromosome complement, C-banding, Ag-NOR and replication banding in the zebrafish Danio rerio. Chromosome Res. 1996; 4:29-32. 
Dai X, Jin X, Chen X, He J, Yin Z. Sufficient numbers of early germ cells are essential for female sex development in zebrafish. PLoS One. 2015; 10:e0117824.

Darwin C R, The Descent of Man, and Selection in Relation to Sex. John Murray, London, 1871.

Devlin RH, Nagahama Y. Sex determination and sex differentiation in fish: an overview of genetic, physiological, and environmental influences. Aquaculture. 2002; 208:191-364.

Dewsbury DA. The Darwin-Bateman paradigm in historical context. Integr. Comp Biol. $2005 ; 45: 831-837$.

Dranow DB, Tucker RP, Draper BW. Germ cells are required to maintain a stable sexual phenotype in adult zebrafish. Dev Biol. 2013; 376:43-50.

Eberhard WG. Postcopulatory sexual selection: Darwin's omission and its consequences. Proc Natl Acad Sci U S A. 2009; 106:10025-32.

Emlen ST, Oring LW. Ecology, sexual selection and the evolution of mating systems. Science. 1977; 197:215-223.

Godwin J. Neuroendocrinology of sexual plasticity in teleost fishes. Front Neuroendocrinol. 2010; 31:203-16.

Gornung E, Gabrielli I, Cataudella S, Sola L. CMA3-banding pattern and fluorescence in situ hybridization with $18 \mathrm{~S}$ rRNA genes in zebrafish chromosomes. Chromosome Res. $1997 ; 5: 40-46$.

Gowaty PA, Hubbell SP. Reproductive decisions under ecological constraints: It's about time. Proc Natl Acad Sci U S A. 2009; 106:10017-10024.

Gowaty PA. Hubbell SP. Chance, time allocation, and the evolution of adaptively flexible sex role behavior. Integr Comp Biol. 2005; 45:931-44.

Gray SM, Dill LM, Tantu FY, Loew ER, Herder F, McKinnon JS. Environment-contingent sexual selection in a colour polymorphic fish. Proc Biol Sci. 2008; 275:1785-91.

Greenfield MD, Rodriguez RL. Genotype-environment interaction and the reliability of mating signals. Anim Behav. 2004; 68:1461-1468.

Guo Y, Cheng H, Huang X, Gao S, Yu H, Zhou R. Gene structure, multiple alternative splicing, and expression in gonads of zebrafish Dmrt1. Biochem Biophys Res Commun. 2005; 330:950-957.

Hare RM, Simmons LW. Sexual selection and its evolutionary consequences in female animals. Biol Rev Camb Philos Soc. 2019; 94:929-956. 
Heule C, Salzburger W, Böhne A. Genetics of sexual development: an evolutionary playground for fish. Genetics. 2014; 196:579-91.

Horth L. Melanic body colour and aggressive mating behaviour are correlated traits in male mosquitofish (Gambusia holbrooki). Proc Biol Sci. 2003; 270:1033-40.

Houwing S, Kamminga LM, Berezikov E, Cronembold D, Girard A, van den Elst H, Filippov DV, Blaser H, Raz E, Moens CB, Plasterk RH, Hannon GJ, Draper BW, Ketting RF. A role for Piwi and piRNAs in germ cell maintenance and transposon silencing in zebrafish. Cell. 2007; 129:69-82.

Howe K, Clark MD, Torroja CF, Torrance J, Berthelot C, Muffato M, Collins JE, Humphray $\mathrm{S}$, et al. The zebrafish reference genome sequence and its relationship to the human genome. Nature. 2013; 496:498-503.

Hsiao CD, Tsai HJ. Transgenic zebrafish with fluorescent germ cell: a useful tool to visualize germ cell proliferation and juvenile hermaphroditism in vivo. Dev Biol. 2003; 262:313-23.

Hubbell SP, Johnson LK. Environmental variance in lifetime mating success, mate choice, and sexual selection. Am Nat. 1987; 130:91-112.

Hunt J, Breuker CJ, Sadowski JA, Moore AJ. Male-male competition, female mate choice and their interaction: determining total sexual selection. J Evol Biol. 2009; 22:13-26.

IPCC Climate Change 2013: The Physical Science Basis. Working Group I Contribution to the Fift Assessment Report of the Intergovernmental Panel on Climate Change. 2013; Cambridge University Press, Cambridge, UK.

Janicke T, Häderer IK, Lajeunesse MJ, Anthes N. Darwinian sex roles confirmed across the animal kingdom. Sci Adv. 2016; 2:e1500983.

Jia FY, Greenfield MD, Collins RD. Genetic variance of sexually selected traits in waxmoths: maintenance by genotype x environment interaction. Evolution. 2000; 54:953-967.

Jones AG, Ratterman NL. Mate choice and sexual selection: what have we learned since Darwin? Proc Natl Acad Sci U S A. 2009; 106 Suppl 1:10001-8.

Kobayashi Y, Nagahama Y, Nakamura M. Diversity and plasticity of sex determination and differentiation in fishes. Sex Dev. 2013; 7:115-125.

Kokko H, Heubel K. Condition-dependence, genotype-by-environment interactions and the lek paradox. Genetica. 2008; 132:209-216.

Kokko H, Jennions MD, Brooks R. Unifying and Testing Models of Sexual Selection. Annu Rev Ecol Evol Syst. 2006; 37:43-66. 
Lawrence C, Ebersole JP, Kesseli RV. Rapid growth and out-crossing promote female development in zebrafish (Danio rerio). Environ Biol Fish. 2008; 81:239-246.

Lee SLJ, Horsfield JA, Black MA, Rutherford K, Fisher A, Gemmell NJ. Histological and transcriptomic effects of $17 \alpha$-methyltestosterone on zebrafish gonad development. BMC Genomics. 2017; 18:557.

Liew WC, Bartfai R, Lim Z, Sreenivasan R, Siegfried KR, Orban L, et al. Polygenic sex determination system in zebrafish. PLoS One. 2012; 7:e34397.

Liew WC, Orbán L. Zebrafish sex: A complicated affair. Brief Funct Genomics. 2014; 13:172-187.

Liu H, Todd EV, Lokman PM, Lamm MS, Godwin JR, Gemmell NJ. Sexual plasticity: A fishy tale. Mol Reprod Dev. 2017; 84:171-194.

Liu W, Li SZ, Li Z, Wang Y, Li XY, Zhong JX, Zhang XJ, Zhang J, Zhou L, Gui JF. Complete depletion of primordial germ cells in an All-female fish leads to Sex-biased gene expression alteration and sterile All-male occurrence. BMC Genomics. 2015; 16:971.

Lo KH, Hui MN, Yu RM, Wu RS, Cheng SH. Hypoxia impairs primordial germ cell migration in zebrafish (Danio rerio) embryos. PLoS One. 2011; 6:e24540.

Luckenbach JA, Borski RJ, Daniels HV, Godwin J. Sex determination in flatfishes: Mechanisms and environmental influences. Semin Cell Dev Biol. 2009; 20:256-63.

Luzio A, Matos M, Santos D, Fontainhas-Fernandes AA, Monteiro SM, Coimbra AM. Disruption of apoptosis pathways involved in zebrafish gonad differentiation by $17 \alpha-$ ethinylestradiol and fadrozole exposures. Aquat Toxicol. 2016; 177:269-284.

Majerus MEN. The genetics and evolution of female choice. Trends Ecol Evol. 1986; 1:1-7.

Manabe H, Ishimura M, Shinomiya A, Sunobe T. Field evidence for bi-directional sex change in the polygynous gobiid fish Trimma okinawae. J Fish Biol. 2007; 70:600609.

Mank JE, Avise JC. Evolutionary diversity and turn-over of sex determination in teleost fishes. Sex Dev. 2009; 3:60-7.

Martin RG. Density dependent aggressive advantage in melanistic male mosquitofish Gambusia affinis holbrooki (Girard). Florida Scient. 1977; 40:393-400.

Nagabhushana A, Mishra RK. Finding clues to the riddle of sex determination in zebrafish. $\mathbf{J}$ Biosci. 2016; 41:145-55. 
Ospina-Alvarez N, Piferrer F. Temperature-dependent sex determination in fish revisited: prevalence, a single sex ratio response pattern, and possible effects of climate change. PLoS One. 2008; 3:e2837.

Pailhoux E, Vigier B, Chaffaux S, Servel N, Taourit S, Furet JP, Fellous M, Grosclaude F, Cribiu EP, Cotinot C, Vaiman D. A 11.7-kb deletion triggers intersexuality and polledness in goats. Nat Genet. 2001; 29:453-458.

Parker GA, Birkhead TR. Polyandry: The history of a revolution. Philos Trans R Soc Lond B Biol Sci. 2013; 368:20120335.

Penman DJ, Piferrer F. Fish Gonadogenesis. Part I: Genetic and Environmental Mechanisms of Sex Determination. Rev Fish Sci. 2008; 16:16-34.

Piferrer F, Ribas L, Díaz N. Genomic approaches to study genetic and environmental influences on fish sex determination and differentiation. Mar Biotechnol. 2012; 14:591-604.

Pijnacker LP, Ferwerda MA. Zebrafish chromosome banding. Genome. 1995; 38:1052-1055.

Pizzari T. et al., Debating sexual selection and mating strategies. Science. 2006; 312:690.

Pradhan A, Khalaf H, Ochsner SA, Sreenivasan R, Koskinen J, Karlsson M, Karlsson J, McKenna NJ, Orbán L, Olsson PE. Activation of NF-kappaB protein prevents the transition from juvenile ovary to testis and promotes ovarian development in zebrafish. J Biol Chem. 2012; 287:37926-38.

Pyron M. Female preferences and male-male interactions in zebrafish (Danio rerio). Can J Zool. 2003; 81:122-125.

Qvarnstrom A. Genotype-by-environment interactions in the determination of the size of a secondary sexual character in the collared flycatcher (Ficedula albicollis). Evolution. $1999 ; 53: 1564-1572$.

Raz E. Primordial germ-cell development: the zebrafish perspective. Nat Rev Genet. 2003; 4:690-700.

Reudink MW, McKellar AE, Marini KL, McArthur SL, Marra PP, Ratcliffe LM. Inter-annual variation in American redstart (Setophaga ruticilla) plumage colour is associated with rainfall and temperature during moult: an 11-year study. Oecologia. 2015;178:161-73.

Ribas L, Liew WC, Díaz N, Sreenivasan R, Orbán L, Piferrer F. Heat-induced masculinization in domesticated zebrafish is family-specific and yields a set of different gonadal transcriptomes. Proc Natl Acad Sci U S A. 2017a; 114:E941-E950. 
Ribas L, Valdivieso A, Díaz N, Piferrer F. On the proper rearing density in domesticated zebrafish to avoid unwanted masculinization. Links with the stress response. J Exp Biol. 2017b; 114:E941-E950.

Richardson BE, Lehmann R. Mechanisms guiding primordial germ cell migration: strategies from different organisms. Nat Rev Mol Cell Biol. 2010;11:37-49.

Rodríguez-Marí A, Cañestro C, BreMiller RA, Nguyen-Johnson A, Asakawa K, Kawakami $\mathrm{K}$, Postlethwait JH. Sex reversal in zebrafish fancl mutants is caused by Tp53-mediated germ cell apoptosis. PLoS Genet. 2010; 6:e1001034.

Rodríguez-Marí A, Wilson C, Titus TA, Canestro C, BreMiller RA, Yan YL, Nanda I, Johnston A, Kanki JP, Gray EM, He X, Spitsbergen J, Schindler D, Postlethwait JH. Roles of brca2 (fancd1) in oocyte nuclear architecture, gametogenesis, gonad tumors, and genome stability in zebrafish. PLoS Genet. 2011; 7:e1001357.

Rodríguez-Marí A, Yan YL, BreMiller RA, Wilson C, Cañestro C, Postlethwait JH. Characterization and expression pattern of zebrafish anti-Müllerian hormone (amh) relative to sox9a, sox9b, and cyp19a1a, during gonad development. Gene Expr Patterns. 2005; 5:655-67.

Roughgarden J, Oishi M, Akçay E. Reproductive social behavior: cooperative games to replace sexual selection. Science. 2006; 311:965-9.

Roughgarden J. in Current Perspectives on Sexual Selection. What's Left After Darwin?. T. Hoquet, Ed. (Springer, Dordrecht, 2015), pp. 85-102.

Sakai Y, Kuniyoshi H, Yoshida M, Fukui Y, Hashimoto H, Gushima K. Social control of terminal phase transition in primary males of the diandric wrasse, Halichoeres poecilopterus (Pisces: Labridae). J Ethol. 2007; 25:57-61.

Santos D, Luzio A, Coimbra AM. Zebrafish sex differentiation and gonad development: A review on the impact of environmental factors. Aquat Toxicol. 2017; 191:141-163.

Shang EH, Yu RM, Wu RS. Hypoxia affects sex differentiation and development, leading to a male-dominated population in zebrafish (Danio rerio). Environ Sci Technol. 2006; 40:3118-22.

Sharma E, Künstner A, Fraser BA, Zipprich G, Kottler VA, Henz SR, Weigel D, Dreyer C. Transcriptome assemblies for studying sex-biased gene expression in the guppy, Poecilia reticulate. BMC Genomics. 2014; 15:400.

Shen ZG, Wang HP. Molecular players involved in temperature-dependent sex determination and sex differentiation in Teleost fish. Genet Sel Evol. 2014; 46:26. 
Siegfried KR, Nüsslein-Volhard C. Germ line control of female sex determination in zebrafish. Dev Biol. 2008; 324: 277-87.

Siegfried KR. In search of determinants: gene expression during gonadal sex differentiation. J Fish Biol. 2010; 76:1879-1902.

Singh AP, Nüsslein-Volhard C. Zebrafish Stripes as a Model for Vertebrate Colour Pattern Formation. Curr Biol. 2015; 25:R81-R92.

Skinner AMJ, Watt PJ. Strategic egg allocation in the zebra fish, Danio rerio. Behav Ecol. 2007; 18:905-909.

Small CM, Carney GE, Mo Q, Vannucci M, Jones AG. A microarray analysis of sex- and gonad-biased gene expression in the zebrafish: evidence for masculinization of the transcriptome. BMC Genomics. 2009; 10:579.

Sreenivasan R, Jiang J, Wang X, Bartfai R, Kwan HY, Christoffels A, Orban L. Gonad differentiation in zebrafish is regulated by the canonical Wnt signaling pathway. Biol Reprod. 2014; 90:45.

Tang-Martinez Z, Ryder TB. The Problem with Paradigms: Bateman's Worldview as a Case Study. Integr Comp Biol. 2005; 45:821-30.

Todd EV, Liu H, Muncaster S, Gemmell NJ. Bending Genders: The Biology of Natural Sex Change in Fish. Sex Dev. 2016; 10:223-241.

Traut W, Winking H. Meiotic chromosomes and stages of sex chromosome evolution in fish: zebrafish, platyfish and guppy. Chromosome Res. 2001; 9:659-672.

Trivers RL. In Sexual Selection and the Descent of Man. B. Campbell, Ed. Aldine Publishing Company, Chicago, 1972; pp. 136-179.

Uchida D, Yamashita M, Kitano T, Iguchi T. An aromatase inhibitor or high water temperature induce oocyte apoptosis and depletion of P450 aromatase activity in the gonads of genetic female zebrafish during sex-reversal. Comp Biochem Physiol A Mol Integr Physiol. 2004; 137:11-20.

Uchida D, Yamashita M, Kitano T, Iguchi T: Oocyte apoptosis during the transition from ovary-like tissue to testes during sex differentiation of juvenile zebrafish. J Exp Biol. 2002; 205:711-718.

Uusi-Heikkilä S, Böckenhoff L, Wolter C, Arlinghaus R. Differential allocation by female zebrafish (Danio rerio) to different-sized males--an example in a fish species lacking parental care. PLoS One. 2012; 7:e48317. 
Valenzuela N, Adams DC, Janzen FJ. Pattern does not equal process: exactly when is sex environmentally determined?. Am Nat. 2003; 161:676-83.

Wang XG, Bartfai R, Sleptsova-Freidrich I, Orban L. The timing and extent of 'juvenile ovary' phase are highly variable during zebrafish testis differentiation. J Fish Biol. 2007; 70:33-44.

Webster KA, Schach U, Ordaz A, Steinfeld JS, Draper BW, Siegfried KR. Dmrt1 is necessary for male sexual development in zebrafish. Dev Biol. 2017; 422:33-46.

West PM, Packer C. Sexual selection, temperature, and the lion's mane. Science. 2002; 297:1339-1343.

West-Eberhard MJ. Darwin's forgotten idea: The social essence of sexual selection. Neurosci Biobehav Rev. 2014; 46:501-8.

Wilson CA, High SK, McCluskey BM, Amores A, Yan YL, Titus TA, Anderson JL, Batzel P, Carvan MJ 3rd, Schartl M, Postlethwait JH. Wild sex in zebrafish: Loss of the natural sex determinant in domesticated strains. Genetics. 2014; 198:1291-1308. 


\section{CHAPTER 2}

\section{Phenotypic plasticity induced using high ambient temperature during embryogenesis in domesticated zebrafish, Danio rerio}

\footnotetext{
Shahrbanou Hosseini ${ }^{1,2}$, Bertram Brenig ${ }^{1,2,3}$, Jens Tetens ${ }^{1,2}$, Ahmad Reza Sharifi ${ }^{1,2}$

${ }^{1}$ University of Goettingen, Department of Animal Sciences, Goettingen, Germany ${ }^{2}$ University of Goettingen, Center for Integrated Breeding Research, Goettingen, Germany ${ }^{3}$ University of Goettingen, Institute of Veterinary Medicine, Goettingen, Germany
}

Published in Reproduction in Domestic Animals, 54(3):435-444 https://doi.org/10.1111/rda.13382 


\section{Contents}

Ambient temperature during early stages of life has a substantial effect on physiological processes, eliciting phenotypic plasticity during zebrafish developmental stages. Zebrafish are known to possess a noteworthy ability to modify their phenotype in dependence of environmental factors. However, there is a poor understanding of the effects of temperature during embryogenesis, which influences the biological functions such as survival ability and masculinization in later developmental stages. Since the middle embryonic phase (pharyngula period) is genetically the most conserved stage in embryogenesis, it is very susceptible to embryonic lethality in developmental processes of vertebrates. Here, we tested the effect of transient perturbations (heat shock) during early development (5-24 hr post-fertilization; hpf) at $35^{\circ} \mathrm{C}$ compare to control group at $28^{\circ} \mathrm{C}$, on survival ability of zebrafish to study the embryonic and post-embryonic mortality. We studied the variation of heat-induced masculinization among and across the families in response to high temperature. Furthermore, morphometric traits of adult zebrafish at different developmental time points were measured in order to estimate the temperature $\times$ sex interaction effect. We found the highest embryonic mortality around the gastrula and segmentation periods in both experimental groups, with significantly lower survival ability in the temperature-treated group $(73.30 \% \pm 0.58 \%$ vs. $70.19 \% \pm 0.57 \%$, respectively). A higher hatching success was observed in the control group $(71.08 \% \pm 0.61 \%)$ compared to the heat-induced group $(67.95 \% \pm 0.60 \%)$. A distinct reduction in survival ability was also observed in both experimental groups during the first two weeks after hatching, followed by a reduced level of changes thereafter. We found sex ratio imbalances across all families, with $25.2 \%$ more males under temperature treatment. Our study on growth performance has shown a positive effect of increased temperature on growth plasticity, with a greater impact on female fish in response to high ambient temperature.

\section{Keywords}

embryogenesis, sex determination, sex ratio, survival ability, temperature, zebrafish 


\section{Introduction}

Sex determination (SD) is a biological process which determines the sexual fate of an organism and controlled varying widely among different animal species. The underlying factors of this mechanism for sexual decision possess diverse cues such as sex chromosomes, environmental effects, social dynamics and the effects of multiple sex-associated genes (Webster et al., 2017). Besides the master switch of SD that typically resides on welldifferentiated sex chromosomes, different environmental factors can override the genetic influence of SD during gonad differentiation in vertebrate species with undifferentiated sex chromosomal pairs (Liew et al., 2012; Liew \& Orban, 2014; Wilson et al., 2014). Zebrafish are widely used as a model animal (Huang et al., 2015; Ribas et al., 2017), and they have no structural differences between the chromosomal sets of the different sexes, and therefore, sex is determined through interaction between the genetic and environment factors $(\mathrm{G} \times \mathrm{E})$ (Liew \& Orban, 2014). A recent study in wild-type and domesticated zebrafish populations, to identify sex-linked polymorphisms, has reported that chromosome 4 is strongly associated with SD in natural populations. Whereas in domesticated populations, no sex-linked loci have been identified, thus suggesting the SD genes are distributed across the genome and sex is determined by a combination of their alleles (Wilson et al., 2014). This type of SD is known as polygenic SD (PSD) (Liew et al., 2012). Temperature is the most common type of environmental stimulation, inducing masculinization in zebrafish and in many closely related species, in which the sex ratio tends to shift in favor of males (Ospina-Alvarez \& Piferrer, 2008; Liew \& Orban, 2014; Ribas et al., 2017).

During embryogenesis in zebrafish, the primordial germ cells (PGCs) form cell clusters at random locations in the early embryos, before gastrulation, during the first four hours of development. The PGCs become motile following specification and migrate towards the somatic gonadal cells, in order to form the primordial gonad, which will then develop into either a testis or an ovary (Richardson \& Lehmann 2010; Liu et al., 2015). This transition completes at the end of the first day of development, whereby the number of PGCs being decisive in determining the sexual fate of the organism (Raz, 2003; Tzung et al., 2015; Liu et al., 2015). In this developmental period, degeneration of PGCs by external environmental stimulations, during cells migration, leads to a decrease in the number of PGCs in the primordial gonad, resulting in masculinization by expression of pro-male genes and inhibition of ovarian developmental genes (Wang \& Orban, 2007; Webster et al., 2017). The influence 
of high water temperature during larval development in the "juvenile ovary" also leads to oocyte apoptosis and decreased activity of the gonadal aromatase genes during gonad transformation, resulting in masculinization in zebrafish (Liew \& Orban, 2014; Ribas et al., 2017).

Furthermore, the effect of environmental stressor in high and low temperature during embryonic development in terms of survival ability leads to an increase of embryonic mortality, reducing the hatching success, which may be due to the embryo malformations and inability of the emerging larvae to break down the eggshell (Aksakal \& Ciltas, 2018). Midembryonic lethality hypothesis predicts an hourglass-like divergence during animal embryogenesis, in which the embryos in the pharyngula period are highly prone to lethality, compared to the other stages, due to the high conservation of gene expression profiles during this developmental stage (Irie \& Kuratani, 2011; Irie \& Kuratani, 2014). A recent study on zebrafish embryonic mortality highlights that the highest level of embryonic mortality happens during the gastrula period (Uchida et al., 2018). Elevated water temperature also influences the larval survival ability after hatching, due to the transition from endogenous to exogenous nutritional sources after depletion of the yolk-sac (Wilson, 2012).

As global climate change is predicted to increase water temperatures, which leads to alterations in the ecology and physiology of wild-type fish species, and increases the risk of extinction in small, inbred populations under natural conditions, due to male-biased sex ratios and temperature-induced mortality (Brown et al., 2015; Dorts et al., 2016; Ribas et al., 2017). The main goals of this study were to investigate the effect of exposure of high ambient temperature during embryogenesis on sex ratio imbalances and survival trajectories of zebrafish during embryonic and post-embryonic development. A further objective of this study was to measure the morphometric traits in different developmental time points of adult zebrafish depending on temperature $\times$ sex interaction effect.

\section{Materials and Methods}

\section{Animal care and husbandry}

The domesticated DDR strain (Von Hertell et al., 1990) of zebrafish was used in this study. All procedures were in strict accordance with the German Animal Welfare Act and national and international recommendations. This study was approved by the University of Goettingen Committee for the care and use of animals (File number E3-17). The fish were kept in 
recirculation systems of aquaculture facilities, according to the approved institutional guidelines on the use of animals for research purposes (Abozaid et al., 2011). The rearing temperature was kept at $28 \pm 0.5^{\circ} \mathrm{C}$, and fish were subjected to a photoperiod of 12-hours day/night rhythm in a mixed sex group. The broodstocks were fed twice daily with industrial dry food (Tetramine Junior, Germany) and freshly hatched Artemia salina nauplii.

\section{Temperature treatments and data recording}

Three consecutive experiments were carried out, during which fertilized eggs from each fullsib family of zebrafish (69 families) were counted and divided into equal proportions in two experimental groups (control and temperature treatment) from single pair mating. The control group was kept at $28^{\circ} \mathrm{C}$, whereas the treatment group was exposed to a high temperature of $35^{\circ} \mathrm{C}$ from 5 to $24 \mathrm{hpf}$. This stage of development is the segmentation stage, which occurs between the gastrula period (50\%-epiboly) and the pharyngula period (Prim-5) (Kimmel et al., 1995; Abozaid et al., 2011). Water temperature in the incubation system was kept constantly at the required temperature and was controlled three times daily using a digital thermometer (Hobby Biotherm Pro, Gelsdorf, Germany) with an accuracy of $\pm 0.1^{\circ} \mathrm{C}$. At the end of treatment period, the temperature of the treated group was gradually reduced to $28^{\circ} \mathrm{C}$, the control temperature. The total numbers of eggs were recorded after fertilization and survival ability of incubated eggs during embryonic development in the pre-treatment ( $5 \mathrm{hpf}$ ) and post-treatment ( $24 \mathrm{hpf}$ ) stages, as well as the hatchability rate (72 hpf), were recorded by counting and removing the number of dead eggs. Embryonic death was defined as the opaque appearance of eggs, collapse of the egg's structure and unhatched eggs. Feeding started at five days post fertilization (dpf), after the yolk sac was absorbed. The larvae were fed three times a day with a commercial food for zebrafish (Tetramine baby, Germany) and Artemia salina nauplii. The larvae were maintained in 3-liter tank (AquaBox® by Aqua Schwarz $\mathrm{GmbH}$, Goettingen, Germany; $23.5 \times 13.5 \times 13 \mathrm{~cm}^{3}$ ) under a photoperiod of 12-hr light and 12-hr darkness with dissolved oxygen around $7 \mathrm{mg} / \mathrm{l}$ and $\mathrm{pH}$ value of water $7.4 \pm 0.2$ which was monitored daily (measured using Multi 3320, Xylem Analytics, GmbH \& Co. KG, WTW, Weilheim, Germany). Other water quality parameters such as ammonia $0.02 \mathrm{mg} / \mathrm{L} \pm$ 0.01 and nitrite $0.1 \mathrm{mg} / \mathrm{L} \pm 0.01$ (measured using Macherey-Nagel Nanocolor 300D, Dueren, Germany) were controlled periodically to ensure that they were within the appropriate range. The animals of one experiment were examined for their post-embryonic survival ability, sex ratio and zootechnical parameters (weight, total and standard length, survival ability) 
measurement until sex maturity. The survival probability of these animals was recorded weekly during the 3 months of age after hatching. The larval survival ability was recorded by counting and removing the number of dead larvae. Zootechnical parameters were measured at 3, 5 and 7 months of age. The phenotypic sex of all individuals in the control and temperature treatment groups was individually determined by the inspection of urogenital papilla. In the case of unclear gonad, the phenotypic sex was determined using microscopic examination. Total and standard lengths of all animals were measured after maturation using a precise digital caliper (Burg-Wächter PRECISE PS 7215, Altenhofer, Germany). For the total length, all measurements included the caudal fin, as there was no caudal fin damage and all individuals exhibited normal morphology. The body mass of mature fish was measured using a digital laboratory scales (Scaltec 120g, Germany).

\section{Statistical analysis}

Statistical analysis of the hatchability of survived eggs was carried out by applying a linear logistic model with a binary response variable, which was modelled as a binomial random variable $\left(\mathrm{y}_{\mathrm{i}}\right)$. The data was then analysed with the GLIMMIX procedure of SAS System 9.3 using the following generalized linear model (Littell et al., 2006):

$\log i t\left[\frac{\pi_{i}}{1-\pi_{i}}\right]=\eta_{i}=\varphi+\alpha_{i}$

where $\pi_{\mathrm{i}}$ is the probability of hatchability at $72 \mathrm{hpf}$ of eggs which survived the pre-treatment stage; $\varphi$ is the overall mean effect; and $\alpha_{\mathrm{i}}$ is the fixed effect of temperature treatment during embryogenesis $\left(\mathrm{i}=1\right.$ : temperature-treated eggs $35^{\circ} \mathrm{C}$ and $\mathrm{i}=2$ : control group $28^{\circ} \mathrm{C}$ ). Least square means were estimated on the logit scale and then back-transformed to the original scale (probability) using the inverse link function $\pi=\exp (\mathrm{x}) /(1+(\mathrm{x}))$, where $\mathrm{x}$ is the least square means estimate underlying logit scale, applying the LSMEANS statement. Significant differences between least square means were tested using a t-test procedure by inclusion of the PDIFF option in the LSMEANS statement and adjusted by Tukey-Kramer correction. Standard errors of least square means were calculated as described by Littell et al. (2006). Embryonic survival ability data at different time points of development were analysed using the same statistical model as described above for hatchability data, where $\pi_{\mathrm{i}}$ is the probability 
of embryonic survival ability at post-treatment stage ( $24 \mathrm{hpf}$ ) of eggs that survived in the pretreatment stage or probability of survival ability at $72 \mathrm{hpf}$ of eggs that were survives during the post-treatment stage of development $(24 \mathrm{hpf})$. Statistical analysis of treatment effect on the sex of adult zebrafish was carried out by applying the same statistical model as described above for hatchability data. The dependent variable $\left(\mathrm{y}_{\mathrm{i}}\right)$ here takes the value 1 for the probability of being male $\pi_{\mathrm{i}}$ or 0 for the probability of being female $1-\pi_{\mathrm{i}}$ for observation i. $\alpha_{\mathrm{i}}$ again represents the fixed effect of temperature treatment during embryogenesis, as described above. The course of larval survival ability during the post-embryonic time periods until 3 months of age for different treatment groups was illustrated according to the Kaplan-Meier method (survival analysis) by using the LIFETEST procedure of SAS System 9.3 (SAS Institute Inc., USA) and using the following model:

$$
\widehat{S}(\mathrm{t})=\prod_{\mathrm{j}: \mathrm{t}_{\mathrm{j}} \leq \mathrm{t}}\left[1-\frac{\mathrm{d}_{\mathrm{j}}}{\mathrm{n}_{\mathrm{j}}}\right] \text { for } \mathrm{t}_{1} \leq \mathrm{t} \leq \mathrm{t}_{\mathrm{k}}
$$

where $\hat{S}(t)$ is the survivor function and $t$ is the lifetime of animal. For each $j t_{j} \geq t$, let $t_{1}<t_{2}<$ $\ldots<\mathrm{t}_{\mathrm{k}}$ representing the different event times. $\mathrm{n}_{\mathrm{j}}$ is the number of individuals at risk just prior to $t_{i}$, and $d_{j}$ is the number of individuals that die at time $t_{j}$ (Allison, 2010).

The impact of treatment, sex and different time points of development (age) on zootechnical parameters were analysed using the GLM procedure of SAS with the following model:

$$
\mathrm{y}_{\mathrm{ijklm}}=\mu+\alpha_{\mathrm{i}}+\beta_{\mathrm{j}}+\lambda_{\mathrm{k}}+\alpha \beta_{\mathrm{ij}}+\alpha \lambda_{\mathrm{ik}}+\beta \lambda_{\mathrm{jk}}+\alpha \beta \lambda_{\mathrm{ijk}}+\delta_{1}+\varepsilon_{\mathrm{ijklm}}
$$

where $y_{\mathrm{ijklm}}$ is the observation for a zootechnical parameter, $\mu$ is the general mean, $\alpha_{\mathrm{i}}$ is the effect of treatment ( $\mathrm{i}$ : $1=$ temperature treatment, $2=$ control), $\beta_{\mathrm{j}}$ is the fixed effect of sex (j:1=male, $2=$ female), $\lambda_{\mathrm{k}}$ the fixed effect of time points of development (k: 1=3 months, $2=5$ month $3=7$ months) $\alpha \beta_{\mathrm{ij}}, \alpha \lambda_{\mathrm{ik}}, \beta \lambda_{\mathrm{jk}}, \alpha \beta \lambda_{\mathrm{ijk}}$ are the fixed effects of interactions, $\delta_{1}$ is the random effect of tank and $\varepsilon_{\mathrm{ijklm}}$ is the random error. 


\section{Results}

\section{Embryonic survival ability and hatchability}

The descriptive statistics of reproduction traits is presented in table 1 . In this study, 12,222 eggs were derived from 69 full-sib families in which a total number of 8,177 larvae were hatched after 72 hpf. A total number of 8,718 eggs were alive during the first day (24 hpf) after temperature treatment. This stage of embryonic development is a critical period due to a series of physiological and morphological changes.

Table 1: The descriptive statistical phenotypic measurements of the fecundity of the broodstocks and viability of zebrafish eggs derived from a total number of 69 families used in this study during embryonic development until hatching.

\begin{tabular}{lcccc}
\hline Variable (time points) & $\begin{array}{c}\text { Means of egg } \\
\text { numbers }\end{array}$ & $\begin{array}{c}\text { Minimum egg } \\
\text { numbers }\end{array}$ & $\begin{array}{c}\text { Maximum egg } \\
\text { numbers }\end{array}$ & $\begin{array}{c}\text { Total egg } \\
\text { numbers }\end{array}$ \\
\hline Fertilized egg number & $177.1 \pm 11$ & 71 & 493 & 12222 \\
Egg number at 5 hpf & $176.3 \pm 11$ & 71 & 491 & 12163 \\
Egg number at 24 hpf & $126.3 \pm 6.4$ & 39 & 295 & 8718 \\
Larvae number at 72 hpf & $123.9 \pm 6.6$ & 37 & 290 & 8177 \\
\hline
\end{tabular}

The survival ability during the life cycles of zebrafish is categorized into two distinct time periods, embryonic and post-embryonic stages, regarding developmental morphologies during the zebrafish development. The morphological and developmental processes of zebrafish embryos at different stages as described by Kimmel et al. (1995) and the period of exposure to high ambient temperatures used in this study are illustrated in Figure 1c. In this study a distinct reduction in survival ability was observed after treatment at $24 \mathrm{hpf}$ in the pharyngula period (Prim-5, White et al., 2017), compared to the survived eggs at the pretreatment $(5 \mathrm{hpf})$ stage in both experimental groups, with a significant $(\mathrm{p}<0.0002)$ lower survival ability in temperature-treated group $(73.30 \% \pm 0.58 \%$ in control vs. $70.19 \% \pm 0.57 \%$ in treated groups). This stage of development is shown to be highly sensitive to fatality independent to elevated water temperature due to high mortality in both treated and nontreated groups (Figure 1a). The hatchability rate was recorded at $72 \mathrm{hpf}$, in which the larvae were swimming free out of their chorion. A significant $(\mathrm{p}<0.0003)$ higher hatching success 
was observed in the control group $(71.08 \% \pm 0.61 \%)$ compared to temperature treatment group $(67.95 \% \pm 0.60 \%)$ based on the living eggs at the pre-treatment stage $(5 \mathrm{hpf})$. This result is mainly a consequence of the number of pre-stage surviving embryos at $24 \mathrm{hpf}$.
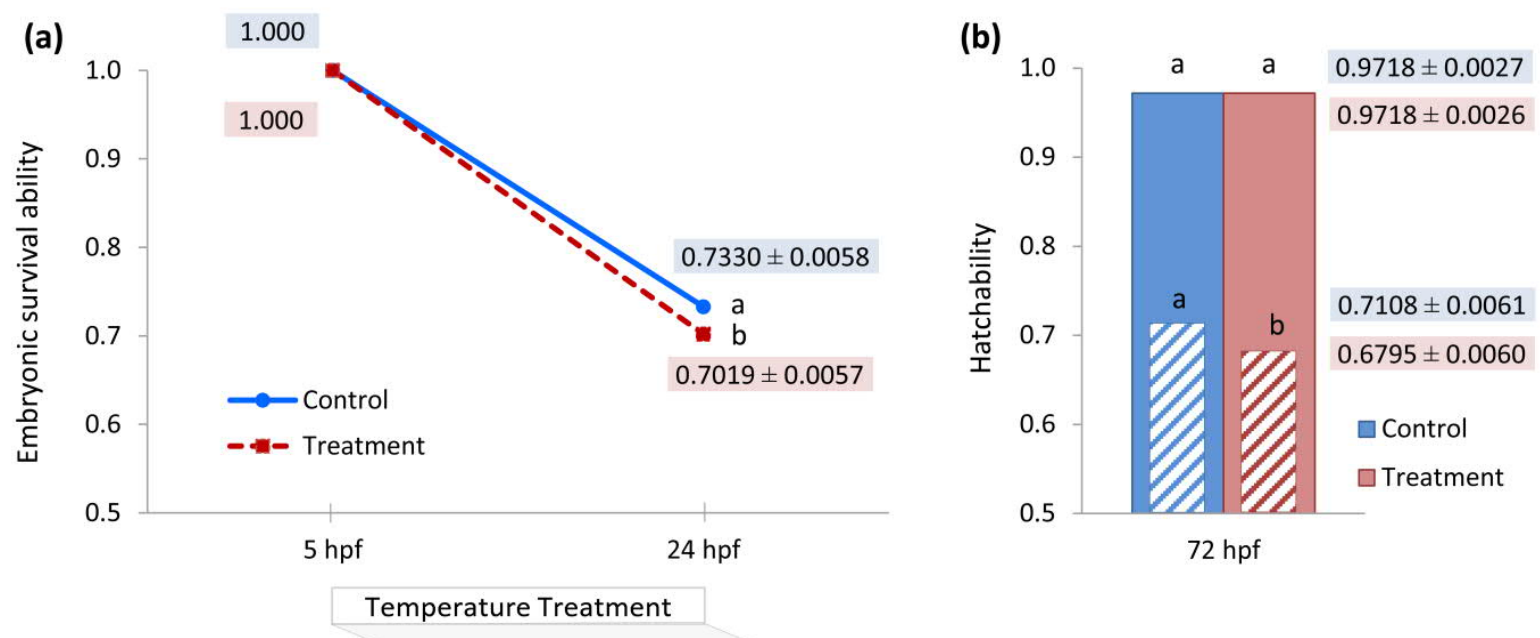

(c)

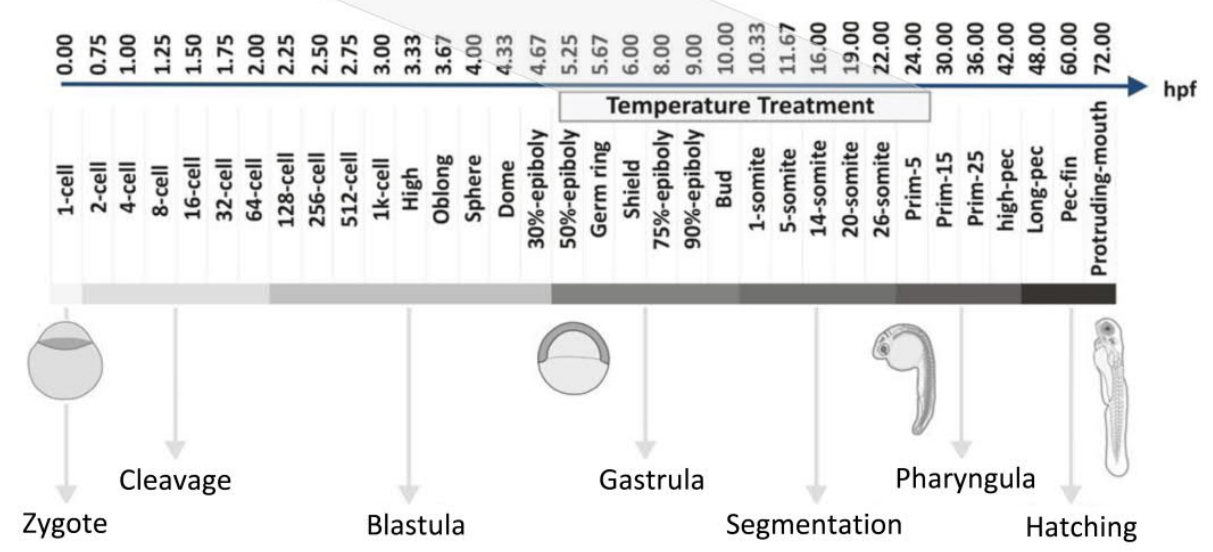

Figure 1: (a) Embryonic survival ability during $24 \mathrm{hr}$ post-fertilization (hpf) of living eggs in the control and temperature-treated groups. (b) Hatchability of larvae at $72 \mathrm{hpf}$ in the control and temperature-treated groups. Pattern-filled bar chart (box filled with diagonal pattern) illustrates the hatchability of larvae based on the living eggs at the pre-treatment stage (5hpf) and the coloured bar chart (solid coloured box) shows the hatchability of larvae on the basis of the total number of survived embryos at 24 hpf. ${ }^{\text {a-b }}$ Back-transformed least square means using generalized linear model within treatment with different superscripts differ significantly $(\mathrm{P}<0.001)$. (c) Schematic diagram explains zebrafish embryonic developmental stages sequentially from stage 1 to 35 as described by Kimmel et al. (1995). 
However, no significant differences in hatching rates were found between the two experimental groups on the basis of the total number of survived embryos at $24 \mathrm{hpf}(97.18 \%$ $\pm 0.27 \%$ in control vs. $97.18 \% \pm 0.26 \%$ in treated groups), meaning that the temperature has no effect on hatchability after treatment period (Figure 1b).

\section{Time-course survival of post-embryonic development}

The effect of elevated water temperature on the survival rate of zebrafish during a series of developmental stages was continuously investigated up to the adult stage (Figure 2). The survival probability, using Kaplan-Meier (KM) survival curves, shows highly significant differences $(\mathrm{P}<0.001)$ between control and temperature-treated groups. The result presented here reveals the obvious decrease in survival ability in both temperature treatment and control groups during the first two weeks post hatching, meaning increased risk of mortality (Hazard rate). Interestingly, after this period, the KM survival curves are illustrated steady lower changes as the number of day increases until $90 \mathrm{dpf}$ in both experimental groups. Despite a constant survival rate in both treated and non-treated groups, we observed a higher mortality in the temperature-treated group, which caused by past survival ability at $14 \mathrm{dpf}$ and lasts until 3 months of age. 


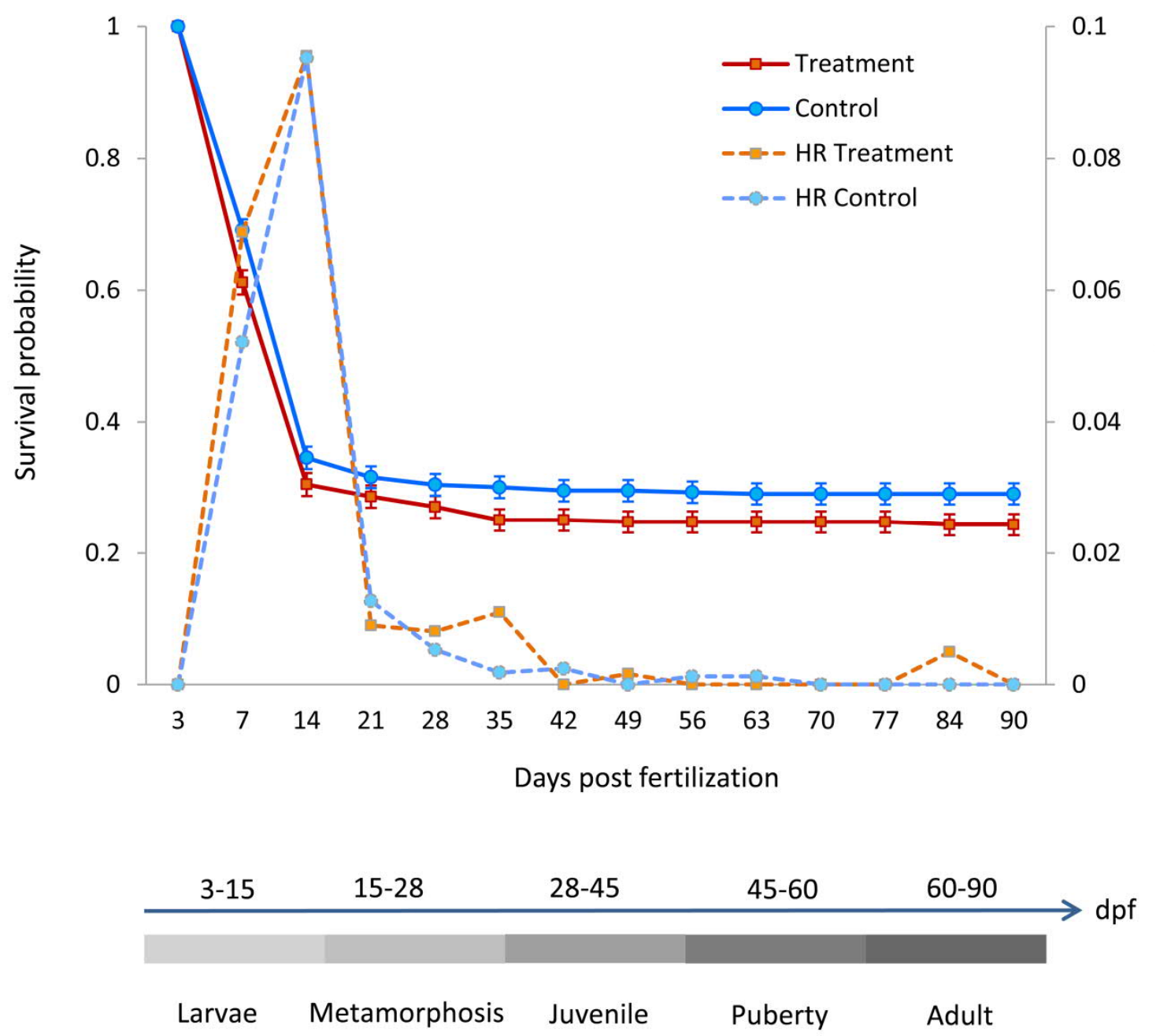

Figure 2: Kaplan-Meier survival curves and hazard rate (HR) illustrate the survival ability of hatched larvae during the post-embryonic development from 3 days post fertilization (dpf) until sexual maturity at $90 \mathrm{dpf}$. The survival curves of different treatment differ significantly using Wilcoxon test $(\mathrm{P}<0.001)$. Post-embryonic developmental stages of zebrafish until adulthood are represented in schematic diagram as defined by Ribas \& Piferrer (2014). 
(a)

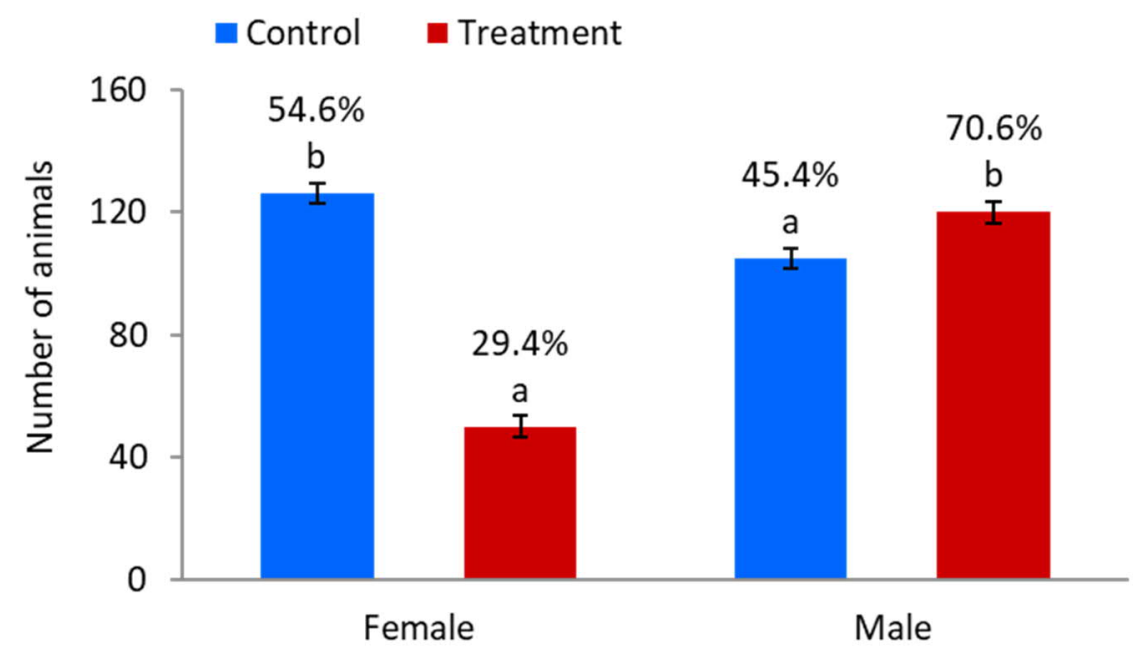

(b)

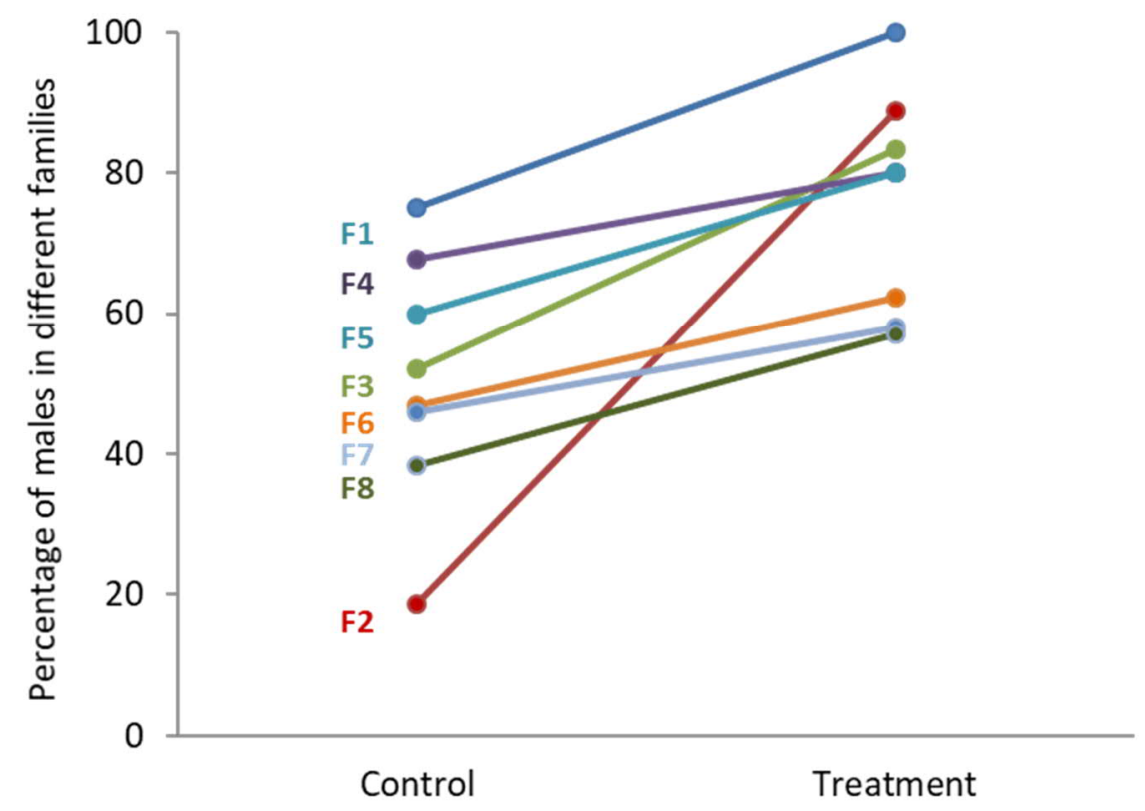

Figure 3: (a) Sex ratio (Back-transformed least square means in \% using generalized linear model) in control and temperature-treated groups across all families. (b) Sex ratio in different families (F) in control and temperature-treated groups (Back-transformed least square means in \% using generalized linear model). ${ }^{\mathrm{a}-\mathrm{b}}$ Means within treatment with different superscripts differ significantly $(\mathrm{P}<0.0001)$. 


\section{Family-specific sex ratio in different temperature treatment}

The sex ratio of adult fish across all families, and family-specific, is shown in Figure 3. The effect of elevated water temperature during embryogenesis indicated a significantly $(\mathrm{p}<0.0001)$ higher proportion of males in the temperature-exposed animals compared to the control group $(70.6 \% \pm 3.49 \%$ vs. $45.4 \% \pm 3.27 \%$, Figure $3 a)$. The results also show a wide range of interfamily sex ratio variation in response to temperature with interplaying between genetic and environmental factors (G×E), implying a PSD-induced masculinization during gonad differentiation in domesticated zebrafish (Figure 3b). A higher male ratio in all families was observed in the temperature-treated groups compared with their corresponding control groups. The effect of increased water temperature is presented in female-biased families (more than 50\% female in the control group) as well as in male-biased families (more than $50 \%$ male in the control group) in family-specific sex ratio result.

\section{Morphometric traits at different time points of zebrafish development}

Table 2 summarizes the least square means and the significance of explanatory variables on body mass, total and standard length of adult zebrafish. For all three variables, a significant effect of the main factors, sex and treatment, as well as an interaction between these main factors was determined. Comparison of the weight of males in treatment and control groups revealed that there is no influence of temperature on the development of body weight $(0.3400$ \pm 0.011 vs. $0.3427 \pm 0.011$ ). However, the temperature treatment caused a significant increase $(\mathrm{p}<0.0001)$ in female body weight, when compared with males, and also compared with the weight of non-treated female animals $(0.5065 \pm 0.013$ females in treated vs. $0.4156 \pm$ 0.011 females in control). A considerably higher body mass was observed in females compared to males $(0.4611 \pm 0.010$ vs. $0.3413 \pm 0.009)$, resulted in significant effect $(\mathrm{p}<0.0001)$ of the main factor sex and treatment. Exposing the embryos to high water temperature, regardless of sex, leads to increased body weight $(0.4232 \pm 0.010$ vs. $0.3791 \pm$ 0.009). The same is true if one looks at means derived from 3-way interactions (sex $\times$ treatment $\times$ age) .

A similar sex $\times$ treatment interaction was found for total and standard length, although no differences were observed between female and male animals in the control group. The highest total and standard length were observed for treated females, when compared with animals of other groups. A significant increase is shown in the total and standard length of 
female animals compared to male animals $(33.98 \pm 0.221$ vs. $33.15 \pm 0.191, p<0.0053 ; 27.74$ \pm 0.203 vs. $27.08 \pm 0.173, \mathrm{p}<0.0131)$ and treatment compared to control $(33.98 \pm 0.222$ vs. $33.15 \pm 0.191, \mathrm{p}<0.0044 ; 27.76 \pm 0.203$ vs. $27.07 \pm 0.173, \mathrm{p}<0.0093)$. At all three age points, the female animals in the treatment group show higher body weight, total and standard lengths compared with animals of other groups, resulting in non-significant three-wayinteractions. This shows that the effect of temperature treatment on weight development and length growth in females begins, and develops more rapidly over the experimental period, in comparison with males which begin at a lower level and do not increase as rapidly as females. This trend can also be observed when looking at the sex $\times$ age interaction, in which the females show a higher weight and length development than the male animals, when the animals become older.

Table 2: Least square means, standard error $( \pm \mathrm{SE})$ and level of significant for body mass $(\mathrm{g})$, total and standard length $(\mathrm{mm})$ in adult zebrafish for the effect of temperature, sex, age and their interactions.

\begin{tabular}{|c|c|c|c|}
\hline \multirow{2}{*}{ Effect } & \multicolumn{3}{|c|}{ Traits } \\
\hline & Body mass (g) & Total length (mm) & Standard length (mm) \\
\hline \multicolumn{4}{|l|}{ Sex } \\
\hline Female $(\mathrm{F})$ & $0.4611 \pm 0.010^{\mathrm{a}}$ & $33.98 \pm 0.221^{\mathrm{a}}$ & $27.74 \pm 0.203^{\mathrm{a}}$ \\
\hline Male (M) & $0.3413 \pm 0.009^{\mathrm{b}}$ & $33.15 \pm 0.191^{\mathrm{b}}$ & $27.08 \pm 0.173^{\mathrm{b}}$ \\
\hline \multicolumn{4}{|c|}{ Temperature treatment } \\
\hline Control (C) & $0.3791 \pm 0.009^{\mathrm{a}}$ & $33.15 \pm 0.191^{\mathrm{a}}$ & $27.07 \pm 0.173^{\mathrm{a}}$ \\
\hline High $(\mathrm{H})$ & $0.4232 \pm 0.010^{\mathrm{b}}$ & $33.98 \pm 0.222^{\mathrm{b}}$ & $27.76 \pm 0.203^{b}$ \\
\hline \multicolumn{4}{|l|}{ Age } \\
\hline 3 months $(3 \mathrm{M})$ & $0.3450 \pm 0.012^{\mathrm{a}}$ & $31.92 \pm 0.281^{\mathrm{a}}$ & $26.31 \pm 0.254^{\mathrm{a}}$ \\
\hline 5 months $(5 \mathrm{M})$ & $0.4030 \pm 0.012^{\mathrm{b}}$ & $33.78 \pm 0.290^{\mathrm{b}}$ & $27.46 \pm 0.262^{b}$ \\
\hline 7 months $(7 \mathrm{M})$ & $0.4555 \pm 0.007^{c}$ & $34.99 \pm 0.172^{\mathrm{c}}$ & $28.47 \pm 0.155^{\mathrm{c}}$ \\
\hline \multicolumn{4}{|l|}{ Sex $\times$ Treatment } \\
\hline $\mathrm{F} \times \mathrm{C}$ & $0.4156 \pm 0.011^{\mathrm{a}}$ & $33.26 \pm 0.276^{\mathrm{a}}$ & $27.12 \pm 0.250^{\mathrm{a}}$ \\
\hline $\mathrm{M} \times \mathrm{C}$ & $0.3427 \pm 0.011^{\mathrm{b}}$ & $33.03 \pm 0.263^{\mathrm{a}}$ & $27.02 \pm 0.238^{\mathrm{a}}$ \\
\hline $\mathrm{F} \times \mathrm{H}$ & $0.5065 \pm 0.013^{c}$ & $34.69 \pm 0.345^{\mathrm{b}}$ & $28.37 \pm 0.312^{b}$ \\
\hline $\mathrm{M} \times \mathrm{H}$ & $0.3400 \pm 0.011^{\mathrm{b}}$ & $33.28 \pm 0.278^{\mathrm{a}}$ & $27.15 \pm 0.252^{\mathrm{a}}$ \\
\hline \multicolumn{4}{|l|}{ Treatment $\times$ Age } \\
\hline $\mathrm{C} \times 3 \mathrm{M}$ & $0.3330 \pm 0.015$ & $31.72 \pm 0.375$ & $26.18 \pm 0.339$ \\
\hline $\mathrm{C} \times 5 \mathrm{M}$ & $0.3809 \pm 0.015$ & $33.27 \pm 0.386$ & $27.04 \pm 0.349$ \\
\hline $\mathrm{C} \times 7 \mathrm{M}$ & $0.4235 \pm 0.008$ & $34.44 \pm 0.194$ & $28.98 \pm 0.176$ \\
\hline $\mathrm{T} \times 3 \mathrm{M}$ & $0.3571 \pm 0.016$ & $32.11 \pm 0.419$ & $26.44 \pm 0.379$ \\
\hline $\mathrm{T} \times 5 \mathrm{M}$ & $0.4251 \pm 0.016$ & $34.30 \pm 0.430$ & $27.87 \pm 0.391$ \\
\hline $\mathrm{T} \times 7 \mathrm{M}$ & $0.4875 \pm 0.011$ & $35.54 \pm 0.284$ & $28.96 \pm 0.256$ \\
\hline
\end{tabular}




\begin{tabular}{|c|c|c|c|}
\hline \multicolumn{4}{|l|}{ Sex $\times$ Age } \\
\hline $\mathrm{F} \times 3 \mathrm{M}$ & $0.3958 \pm 0.015^{\mathrm{bc}}$ & $32.39 \pm 0.402$ & $26.74 \pm 0.363$ \\
\hline $\mathrm{F} \times 5 \mathrm{M}$ & $0.4491 \pm 0.017^{\mathrm{b}}$ & $33.99 \pm 0.447$ & $27.54 \pm 0.404$ \\
\hline $\mathrm{F} \times 7 \mathrm{M}$ & $0.5382 \pm 0.011^{\mathrm{a}}$ & $35.55 \pm 0.282$ & $28.95 \pm 0.254$ \\
\hline $\mathrm{M} \times 3 \mathrm{M}$ & $0.2942 \pm 0.015^{\mathrm{d}}$ & $31.44 \pm 0.393$ & $25.88 \pm 0.355$ \\
\hline $\mathrm{M} \times 5 \mathrm{M}$ & $0.3569 \pm 0.014^{\mathrm{c}}$ & $33.58 \pm 0.370$ & $27.38 \pm 0.334$ \\
\hline $\mathrm{M} \times 7 \mathrm{M}$ & $0.3728 \pm 0.008^{c}$ & $34.44 \pm 0.197$ & $28.00 \pm 0.178$ \\
\hline \multicolumn{4}{|l|}{ Sex $\times$ Treatment $\times$ Age } \\
\hline $\mathrm{F} \times \mathrm{C} \times 3 \mathrm{M}$ & $0.3662 \pm 0.020$ & $31.73 \pm 0.544$ & $26.16 \pm 0.491$ \\
\hline $\mathrm{F} \times \mathrm{C} \times 5 \mathrm{M}$ & $0.4025 \pm 0.021$ & $33.22 \pm 0.575$ & $26.87 \pm 0.500$ \\
\hline $\mathrm{F} \times \mathrm{C} \times 7 \mathrm{M}$ & $0.4780 \pm 0.010$ & $34.83 \pm 0.250$ & $28.31 \pm 0.226$ \\
\hline $\mathrm{F} \times \mathrm{T} \times 3 \mathrm{M}$ & $0.4255 \pm 0.022$ & $33.06 \pm 0.593$ & $27.32 \pm 0.536$ \\
\hline $\mathrm{F} \times \mathrm{T} \times 5 \mathrm{M}$ & $0.4958 \pm 0.025$ & $34.76 \pm 0.684$ & $28.21 \pm 0.618$ \\
\hline $\mathrm{F} \times \mathrm{T} \times 7 \mathrm{M}$ & $0.5984 \pm 0.018$ & $36.26 \pm 0.505$ & $29.59 \pm 0.457$ \\
\hline $\mathrm{M} \times \mathrm{C} \times 3 \mathrm{M}$ & $0.2997 \pm 0.020$ & $31.72 \pm 0.517$ & $26.19 \pm 0.467$ \\
\hline $\mathrm{M} \times \mathrm{C} \times 5 \mathrm{M}$ & $0.3593 \pm 0.019$ & $33.32 \pm 0.517$ & $27.21 \pm 0.467$ \\
\hline $\mathrm{M} \times \mathrm{C} \times 7 \mathrm{M}$ & $0.3690 \pm 0.012$ & $34.05 \pm 0.298$ & $27.65 \pm 0.270$ \\
\hline $\mathrm{M} \times \mathrm{T} \times 3 \mathrm{M}$ & $0.2888 \pm 0.022$ & $31.17 \pm 0.592$ & $25.57 \pm 0.536$ \\
\hline $\mathrm{M} \times \mathrm{T} \times 5 \mathrm{M}$ & $0.3544 \pm 0.020$ & $33.84 \pm 0.530$ & $27.54 \pm 0.479$ \\
\hline $\begin{array}{l}M \times 1 \times 5 M \\
M \times T \times 7 M\end{array}$ & $0.3766 \pm 0.010$ & $34.82 \pm 0.258$ & $28.33 \pm 0.233$ \\
\hline ANOVA, $P$-value $(F)$ & Weight & Total length & Standard length \\
\hline Sex & $\leq 0.0001$ & $\leq 0.0053$ & $\leq 0.0131$ \\
\hline Temperature treatment & $\leq 0.0001$ & $\leq 0.0044$ & $\leq 0.0093$ \\
\hline Age & $\leq 0.0001$ & $\leq 0.0001$ & $\leq 0.0001$ \\
\hline Sex $\times$ Treatment & $\leq 0.0001$ & $\leq 0.0441$ & $\leq 0.0339$ \\
\hline Treatment $\times$ Age & $\leq 0.2305$ & $\leq 0.5511$ & $\leq 0.4887$ \\
\hline Sex $\times$ Age & $\leq 0.0018$ & $\leq 0.5804$ & $\leq 0.4228$ \\
\hline Sex $\times$ Treatment $\times$ Age & $\leq 0.6699$ & $\leq 0.6549$ & $\leq 0.6084$ \\
\hline
\end{tabular}

${ }^{\mathrm{a}-\mathrm{d}}$ Means within a factor with different superscripts differ $(\mathrm{P}<0.05)$.

\section{Discussion}

\section{Embryonic and post-embryonic survival ability}

In this study, the effect of high ambient temperature during embryogenesis on reproductive, survival ability, sex ratio imbalances and morphometric traits during zebrafish developmental stages were investigated. The fecundity of zebrafish is relatively low, compared to other fish species, with a maximum of 500 eggs per clutch due to their small size. A larger clutch size of laboratory zebrafish females was observed than wild population (Ribas \& Piferrer, 2014), which is in agreement with the fecundity ability of laboratory strain used in this study. 
The first stage of development (1.5 hpf) is the fourth phase of cleavage and produces a set of $4 \times 4$ arrays of blastomeres cells (16-cells) in the zygote. However, in the second developmental period ( $5 \mathrm{hpf}$ ), epiboly displays blastoderm margin between the animal and vegetal pole. In the pharyngula period, a variety of morphogenetic movements occur and the primary organs become visible, in particular, the tail bud becomes increasingly distinguished and the first body movements appear (Kimmel et al., 1995). In evolutionary developmental biology, the hourglass model of embryonic evolution in vertebrates predicted the midembryonic phase, namely the phylotypic period, to be the most conserved organogenesis stage compare to earliest and latest stages of embryo development. Transcriptome profile during mid-embryonic phase (pharyngula period) in vertebrates is highly conserved, which provides a gene source for the development of the basic body plan (Irie \& Kuratani, 2011; Irie \& Kuratani, 2014). Therefore, it was hypothesized that increased embryonic mortality would potentially occur during this conserved developmental period (Irie \& Kuratani, 2011; Uchida et al., 2018). However, in the study completed by Uchida et al. (2018), the embryonic mortality was higher in earlier stages of development around the gastrula and early segmentation periods, which is in agreement with the results of our study. In the same study, increased embryonic mortality was observed during the gastrula stage with high-temperature treatment as a thermal perturbation, emphasizing the sensitivity of these stages to environmental factors (Uchida et al., 2018). In a comparable study, carried out on eggs deriving from the mating of mitotic gynogenic male with normal female, a low hatching rate at 72 hpf was reported in temperature-induced zebrafish compared to control group (16\% vs. $27 \%)$ that were treated by high water temperature $\left(35^{\circ} \mathrm{C}\right)$ at $5-24 \mathrm{hpf}$ during embryogenesis. However, there is no information provided from the authors regarding embryonic survival ability during previous stages of development, before the hatching process started (Abozaid et al., 2011). A pronounced adverse effect of increased water temperature as a perturbation factor in the embryogenesis process leading to increased mortality before 24 hpf was also observed in the study of Aksakal \& Ciltas (2018).

Cooling exposure at 50\% epiboly stage for 6 and $18 \mathrm{hr}$ in zebrafish embryos also resulted in a considerably reduced embryonic survival ability (control: $67.50 \%$ vs. $18 \mathrm{hr}: 39.80 \%$ and $6 \mathrm{hr}$ : $18.75 \%$ ) during hatching process (Paes \& Nakaghi, 2017). From the embryos surviving the gastrulation stage, a further reduction in hatchability in the post-gastrulation stage, under the influence of high water temperature, may be due to the inhibition of mitosis, the inhibition of the hatching enzyme activity, suppression of embryogenesis or an inability of the emerging 
larvae to break down the eggshell along with malformations such as pericardial and yolk-sac edema, tail deformity and spinal curvature (Aksakal \& Ciltas, 2018).

The early larvae stage in zebrafish lasts up to two weeks following hatching ( $15 \mathrm{dpf}$; Ribas \& Piferrer, 2014), involving developmental stages such as yolk-sac larvae ( 4 dpf) and swimup larvae ( 9 dpf) characterized by physiological and morphological changes (Bagatto et al., 2001). The swim bladder inflates during 4-5 dpf and they can start short burst swimming, whereas the digestive tract opens and the digestive enzymes are secreted during the 5-6 dpf, suggesting the larvae can feed exogenously from endogenous feeding (Wilson, 2012). Larvae are more vulnerable during these developmental stages due to the depletion of yolk-sac, inflation of the swimming bladder and swimming performance influencing the food capture, (Sfakianakis et al., 2011; Wilson, 2012). Furthermore, the early life temperature treatment could have an effect on fish muscle ontogeny or body shape (Sfakianakis et al., 2011), which impacts the swimming ability in water current direction. The larval stage (takes up to 2 weeks), involves changes in a wide range of traits such as development of scales, pigmentation patterns and gut tube drops more ventrally (Ribas \& Piferrer, 2014). This wide range of morphological and physiological changes might be the cause of increased mortality in this stage of development, which is observed in our study, in particular in the case of treated-animals. In the later stage of development, free-swimming larvae ( $21 \mathrm{dpf}$ : Mid-stage larvae, Bagatto et al., 2001; Wilson, 2012) start metamorphosis between 15 and 28 dpf. In our study, this developmental process has no particular effect on larval survival ability.

\section{Sex ratio response to environmental temperature}

In this study, the effect of elevated temperature during early zebrafish life resulted in sex ratio imbalances with a higher proportion of males in heat-exposure animals. A similar study of elevated water temperature on the golden strain of zebrafish during different embryonic developmental stages (5-10, 5-24 and 5-48 hpf) at $35^{\circ} \mathrm{C}$ in half-sib families derived from mating of mitotic gynogenic males with normal females, resulted in a higher proportion of males by $25 \%$ in $5-24$ hpf ( $22 \%$ in control vs. $47.54 \%$ in treatment), although a higher proportion of females in the progeny generation was expected (Abozaid et al., 2011). The results reported in this study, in terms of masculinization, are in agreement with our study.

Heat-induced masculinization due to temperature treatment at the larval stage, between 18 and $32 \mathrm{dpf}$ in the $\mathrm{AB}$ strain of domesticated zebrafish, is also showed that the sex ratio varies considerably among different families (Ribas et al., 2017). The result of the study of Ribas et 
al. (2017) revealed that some heat-treated animals altered their sex from genotypic females to phenotypic males, these individuals being known as "neomales", and the remaining genotypic females can be considered as heat-resistant individuals or "superfemales". Neomales possess testis and have similar gene expression profiles to normal males, but the underlying genetic mechanism for SD of these animals and the heat-resistant females is so far unknown (Ribas et al., 2017). Therefore, the result of our study in PSD confirms previous observations of environmental dependency of sexual plasticity in domesticated zebrafish. Phenotypic masculinization is also reported in many other fish species with similar SD plasticity, such as European sea bass, Dicentrarchus labrax (Díaz \& Piferrer, 2015), medaka, Oryzias latipes (Selim et al., 2009), and Nile tilapia, Oreochromis niloticus (Rougeot et al., 2008).

PGCs play an important role in gonad differentiation and sexual dimorphism (Tzung et al., 2015; Liu et al., 2015). Elevated water temperature is the most important environmental parameter, may induce degeneration of PGCs, influencing the genetic mechanism of SD, resulting in masculinization (Lee et al., 2009; Selim et al., 2009). The genetic regulation of SD in juvenile ovaries during gonad development and sexual polymorphism is controlled by the major pro-male (e.g. dmrt1, amh, cyllc1) and pro-female genes (e.g. cyp19ala, foxl2, $v t g 2)$. In the masculinization process, expression of $d m r t 1$, a key regulator of SD gene in males, activates amh, which inhibits the ovarian aromatase gene expression and oocyte apoptosis, resulting in ovary-to-testis transformation (Wang \& Orban, 2007; Webster et al., 2017; Lee et al., 2017). In this process, upregulation of the most important pro-male pathway (Tp53-activated apoptosis) and downregulation of pro-female pathways (NF- $\kappa \mathrm{B}$ and canonical Wnt) caused a shift of the sex ratio towards an increased proportion of males (Liew \& Orban, 2014).

\section{Morphometric traits}

High and low temperatures $\left(32^{\circ} \mathrm{C}\right.$ and $22^{\circ} \mathrm{C}$ compared to the control at $\left.27^{\circ} \mathrm{C}\right)$ during embryonic development in zebrafish, have an influence on the acclimation response, which has an influence on energy metabolism and swimming performance in later life stages, which is controlled by expression of several metabolic genes (Scott \& Johnston, 2012) in swimming muscle (Schnurr et al., 2014). In the study of Schnurr et al. (2014), temperature treatment during early life in zebrafish resulted in a reduction of body mass and standard length until 12 weeks of age. However, in the high-temperature group, a significant growth rate was 
observed beyond this age and the body length was higher than the control group (Schnurr et al., 2014), which is in agreement with our study. The influence of different temperature ranges during embryonic development on post-hatching growth rate in other fish species has also been reported (Macqueen et al., 2008). The effect of different temperature (low temperature: $18^{\circ} \mathrm{C}$, high temperature: $22^{\circ} \mathrm{C}$ ) during embryogenesis on muscle growth rate and body mass in gilthead sea bream showed that early temperature treatment has an influence on the expression patterns of a subset of muscle developmental genes (Hsp90a, UNC45, MyoD and $I G F 1$ ) and their expression is modified by different temperature regimes (Serrana et al., 2012). Interestingly, a positive effect of elevated water temperature on growth rate was observed during post-embryonic development when the animals were reared at three different temperatures: $24^{\circ} \mathrm{C}, 28.5^{\circ} \mathrm{C}$ and $33^{\circ} \mathrm{C}$. Animals reared at $24^{\circ} \mathrm{C}$ grew slower than those reared at $33^{\circ} \mathrm{C}$ (Parichy et al., 2009). Our study also demonstrated a positive effect of increased temperature on growth performance, with a higher influence on females in response to high ambient temperature.

\section{Conclusion}

Exposure to high water temperature during embryonic development in domesticated zebrafish demonstrated the reduction of survival ability in the first day after fertilization and the first two weeks after hatching, which shows the most sensitive life stages to thermal changes in the environment. Furthermore, the effect of elevated water temperature during embryogenesis resulted in sex ratio imbalances with more males under high-temperature condition. Our study on growth performance in different developmental life cycles in heat-induced domesticated zebrafish during adult stages after sexual maturity has shown the growth plasticity in response to high ambient temperature for different sexes. A positive effect of increased temperature during embryogenesis on growth with a greater impact in female fish is observed in this study.

\section{Acknowledgements}

The authors are grateful of the Aquaculture and Aquatic Ecology staffs to provide the possibility to use the aquaculture facilities and animals. 


\section{Conflict of interest}

The authors declare that no conflicting personal or financial interests.

\section{Author contributions}

S.H. contributed to the conception and design of the study, carried out the experiments, interpreted the results and wrote the manuscript. A.R.S. designed the study and conception. S.H. and A.R.S. performed computational data analysis and interpreted the data. B.B., J.T. and A.R.S. edited and corrected the manuscript. All authors read and commented on the manuscript and approved the final version.

\section{References}

Abozaid H, Wessels S, Hörstgen-Schwark G. Effect of rearing temperatures during embryonic development on the phenotypic sex in zebrafish (Danio rerio). Sex Dev. 2011; 5:259-265.

Aksakal FI, Ciltas A. The impact of ultraviolet B (UV-B) radiation in combination with different temperatures in the early life stage of zebrafish (Danio rerio). Photochem Photobiol Sci. 2018; 17:35-41.

Allison PD. Survival Analysis Using SAS: A Practical Guide, (2nd ed.). Cary, NC, SAS Institute Inc, 2010.

Bagatto B, Pelster B, Burggren WW. Growth and metabolism of larval zebrafish: effects of swim training. J Exp Biol. 2001; 204:4335-43.

Brown AR, Owen SF, Peters J, Zhang Y, Soffker M, Paull GC, Hosken DJ, Wahab MA, Tyler CR. Climate change and pollution speed declines in zebrafish populations. Proc Natl Acad Sci U S A. 2015; 112:E1237-E1246.

Díaz N, Piferrer F. Lasting effects of early exposure to temperature on the gonadal transcriptome at the time of sex differentiation in the European sea bass, a fish with mixed genetic and environmental sex determination. BMC Genomics. 2015; 16:679.

Dorts J, Falisse E, Schoofs E, Flamion E, Kestemont P, Silvestre F. DNA methyltransferases and stress-related genes expression in zebrafish larvae after exposure to heat and copper during reprogramming of DNA methylation. Sci Rep. 2016; 6:34254.

Huang Y, Wang XL, Zhang JW, Wu KS. Impact of endocrine-disrupting chemicals on reproductive function in zebrafish (Danio rerio). Reprod Domest Anim. 2015; 50:1-6. 
Irie N, Kuratani S. Comparative transcriptome analysis reveals vertebrate phylotypic period during organogenesis. Nat Commun. 2011; 2:248.

Irie N, Kuratani S. The developmental hourglass model: a predictor of the basic body plan?. Development. 2014; 141:4649-55.

Kimmel CB, Ballard WW, Kimmel SR, Ullmann B, Schilling TF. Stages of embryonic development of the zebrafish. Dev Dyn. 1995; 203:253-310.

Lee KH, Yamaguchia A, Rashida H, Kadomura K, Yasumoto S, Matsuyama M. Germ cell degeneration in high-temperature treated Pufferfish, Takifugu rubripes. Sex Dev. 2009; 3:225-32.

Lee, SLJ, Horsfield JA, Black MA, Rutherford K, Fisher A, Gemmell NJ. Histological and transcriptomic effects of $17 \alpha$-methyltestosterone on zebrafish gonad development. BMC Genomics. 2017; 18:557.

Liew WC, Bartfai R, Lim Z, Sreenivasan R, Siegfried KR, Orban L. Polygenic sex determination system in zebrafish. PLoS One. 2012; 7:e34397.

Liew WC, Orbán L. Zebrafish sex: A complicated affair. Brief Funct Genomics. 2014; 13:172-187.

Littell RC, Milliken GA, Stroup WW, Wolfinger RD. SAS system for Mixed Models: Cary, NC, SAS Institute Inc. 633 pp, 2006.

Liu W, Li SZ, Li Z, Wang Y, Li XY, Zhong JX, Zhang XJ, Zhang J, Zhou L, Gui JF. Complete depletion of primordial germ cells in an All-female fish leads to Sex-biased gene expression alteration and sterile All-male occurrence. BMC Genomics. 2015; 16:971-986.

Macqueen DJ, Robb DH, Olsen T, Melstveit L, Paxton CG, Johnston IA. Temperature until the 'eyed stage' of embryogenesis programmes the growth trajectory and muscle phenotype of adult Atlantic salmon. Biol Lett. 2008; 4:294-8.

Ospina-Alvarez N, Piferrer F. Temperature-dependent sex determination in fish revisited: prevalence, a single sex ratio response pattern, and possible effects of climate change. PLoS One. 2008; 3:e2837.

Paes MDCF, Nakaghi LSO. Post-cooling survival, growth and deformity rates in zebrafish embryos (Danio rerio). Zygote. 2017; 26:76-88.

Parichy DM, Elizondo MR, Mills MG, Gordon TN, Engeszer RE. Normal table of postembryonic zebrafish development: staging by externally visible anatomy of the living fish. Dev Dyn. 2009; 238:2975-3015. 
Raz E. Primordial germ-cell development: the zebrafish perspective. Nat Rev Genet. 2003; 4:690-700.

Ribas L, Liew WC, Díaz N, Sreenivasan R, Orbán L, Piferrer F. Heat-induced masculinization in domesticated zebrafish is family-specific and yields a set of different gonadal transcriptomes. Proc Natl Acad Sci U S A. 2017; 114:E941-E950.

Ribas L, Piferrer F. The zebrafish (Danio rerio) as a model organism, with emphasis on applications for finfish aquaculture research. Rev Aquacult. 2014; 6:209-240.

Richardson BE, Lehmann R. Mechanisms guiding primordial germ cell migration: strategies from different organisms. Nat Rev Mol Cell Biol. 2010; 11:37-49.

Rougeot C, Prignon C, Kengne CVN, Mélard C. Effect of high temperature during embryogenesis on the sex differentiation process in the Nile tilapia, Oreochromis niloticus. Aquaculture. 2008; 276:205-208.

Schnurr ME, Yin Y, Scott GR. Temperature during embryonic development has persistent effects on metabolic enzymes in the muscle of zebrafish. J Exp Biol. 2014; 217:137080.

Scott GR, Johnston IA. Temperature during embryonic development has persistent effects on thermal acclimation capacity in zebrafish. Proc Natl Acad Sci U S A. 2012; 109:1424752.

Selim KM, Shinomiya A, Otake H, Hamaguchi S, Sakaizumi M. Effects of high temperature on sex differentiation and germ cell population in medaka, Oryzias latipes. Aquaculture. 2009; 289:340-349.

Serrana DGDI, Vieira VL, Andree KB, Darias M, Estévez A, Gisbert E, Johnston IA. Development temperature has persistent effects on muscle growth responses in gilthead sea bream. PLoS One. 2012; 7:e51884.

Sfakianakis DG, Leris I, Kentouri M. Effect of developmental temperature on swimming performance of zebrafish (Danio rerio) juveniles. Environ Biol Fish. 2011; 90:421427.

Tzung KW, Goto R, Saju JM, Sreenivasan R, Saito T, Arai K, Yamaha E, Hossain MS, Calvert ME, Orbán L. Early depletion of primordial germ cells in zebrafish promotes testis formation. Stem Cell Reports. 2015; 4:61-73.

Uchida Y, Uesaka M, Yamamoto T, Takeda H, Irie N. Embryonic lethality is not sufficient to explain hourglass-like conservation of vertebrate embryos. Evodevo. 2018; 9:7. 
Von Hertell U, Hörstgen-Schwark G, Langholz HJ, Jung B. Family studies on genetic variability in growth and reproductive performance between and within test fish populations of the zebrafish, Brachydanio rerio. Aquaculture. 1990; 85:307-315.

Wang X, Orban L. Anti-Müllerian hormone and 11 beta-hydroxylase show reciprocal expression to that of aromatase in the transforming gonad of zebrafish males. Dev Dyn. 2007; 236:1329-38.

Webster KA, Schach U, Ordaz A, Steinfeld JS, Draper BW, Siegfried KR. Dmrt1 is necessary for male sexual development in zebrafish. Dev Biol. 2017; 422:33-46.

White RJ, Collins JE, Sealy IM, Wali N, Dooley CM, Digby Z, Stemple DL, Murphy DN, Billis K, Hourlier T, Füllgrabe A, Davis MP, Enright AJ, Busch-Nentwich EM. A highresolution mRNA expression time course of embryonic development in zebrafish. Elife. 2017; 6:e30860.

Wilson C. Aspects of larval rearing. ILAR J. 2012; 53:169-78.

Wilson CA, High SK, McCluskey BM, Amores A, Yan YL, Titus TA, Anderson JL, Batzel P, Carvan MJ, Schartl M, Postlethwait JH. Wild sex in zebrafish: Loss of the natural sex determinant in domesticated strains. Genetics. 2014; 198:1291-1308. 


\title{
CHAPTER 3
}

\section{Efficient phenotypic sex classification of zebrafish using machine learning methods}

\author{
Shahrbanou Hosseini ${ }^{1,2}$, Henner Simianer ${ }^{1,2}$, Jens Tetens ${ }^{1,2}$, Bertram Brenig ${ }^{1,2,3}$, \\ Sebastian Herzog ${ }^{4,5}$, Ahmad Reza Sharifi ${ }^{1,2}$ \\ ${ }^{1}$ University of Goettingen, Department of Animal Sciences, Goettingen, Germany \\ ${ }^{2}$ University of Goettingen, Center for Integrated Breeding Research, Goettingen, Germany \\ ${ }^{3}$ University of Goettingen, Institute of Veterinary Medicine, Goettingen, Germany \\ ${ }^{4}$ Max Planck Institute for Dynamics and Self-Organization, Goettingen, Germany \\ ${ }^{5}$ University of Goettingen, Department for Computational Neuroscience, 3rd Physics Institute- \\ Biophysics, Goettingen, Germany
}

Published in Ecology and Evolution, 2019

https://doi.org/10.1002/ece3.5788 


\section{Abstract}

Sex determination in zebrafish by manual approaches according to current guidelines relies on human observation. These guidelines for sex recognition have proven to be subjective and highly labor-intensive. To address this problem, we present a methodology to automatically classify the phenotypic sex using two machine learning methods: Deep Convolutional Neural Networks (DCNNs) based on the whole fish appearance and Support Vector Machine (SVM) based on caudal fin coloration. Machine learning techniques in sex classification provide potential efficiency with the advantage of automatisation and robustness in the prediction process. Furthermore, since developmental plasticity can be influenced by environmental conditions, we have investigated the impact of elevated water temperature during embryogenesis on sex and sex-related differences in color intensity of adult zebrafish. The estimated color intensity based on SVM was then applied to detect the association between coloration and body weight and length. Phenotypic sex classifications using machine learning methods resulted in a high degree of association with the real sex in non-treated animals. In temperature-induced animals, DCNNs reached a performance of $100 \%$, whereas $20 \%$ of males were misclassified using SVM due to a lower color intensity. Furthermore, a positive association between color intensity and body weight and length was observed in males. Our study demonstrates that high ambient temperature leads to a lower color intensity in male animals and a positive association of male caudal fin coloration with body weight and length, which appears to play a significant role in sexual attraction. The software developed for sex classification in this study is readily applicable to other species with sex-linked visible phenotypic differences.

Keywords: color, machine learning, sex classification, temperature, zebrafish

\section{Introduction}

Sexual dimorphism between males and females such as sexually dichromatism can play an important role in sexual attraction for a potential mate (Singh \& Nüsslein-Volhard, 2015). Sexual attraction with respect to color has been observed in some fish species in previous studies (Gronell, 1989; Kraak et al., 1999): Mating preference in female Chrysiptera cyanea is correlated with intensity of orange caudal fin coloration in males during courtship periods, and reproductive success in Gasterosteus aculeatus increased with redness of male throat. 
Coloration can therefore be used in sexually dichromatic species for sex determination. Zebrafish have emerged as a well-established vertebrate model organism for studies of biology, genetics, embryonal development, diseases, drug screening and environmental effect (Nowik et al., 2015; Ribas et al., 2017). This animal possesses sexual plasticity, meaning that it changes its sex depending on environmental factors (Kobayashi et al., 2013), which makes it a suitable organism for studying the environmental effects on phenotypic plasticity in fish populations. Temperature is one of the most important environmental factors affecting the animal's phenotype. Previous studies reported that high temperature leads to an increase in the proportion of males in zebrafish (Abozaid et al., 2011; Ribas et al., 2017; Hosseini et al., 2019a; Hosseini et al., 2019b). The sex ratio can influence population dynamics leading to a reduction in genetic variation and loss of heterozygosity, which increases inbreeding and adversely affects fitness traits resulting in the risk of extinction, particularly in small and isolated populations (Brown et al., 2015). Furthermore, a recent study demonstrated that the effect of high temperature in heat-exposed zebrafish leads to a loss of pigmentation intensity (Ribas et al., 2017). Generally, the intensity of color in fish is regulated by genetic factors and controlled through neurohormonal mechanisms (Price et al., 2008; Hutter et al., 2012). Beyond the genetic regulation of color intensity, variation in coloration can be influenced by environmental factors such as temperature and light (Price et al., 2008).

In zebrafish, males show a slightly more intense yellow coloration compared to females, which is thought to be important for sexual attraction (Hutter et al., 2010; Singh \& NüssleinVolhard, 2015; Nüsslein-Volhard \& Singh, 2017). Furthermore, previous studies showed that female zebrafish are able to visually discriminate sexes, that is, they recognize males based on their yellow coloration, particularly during courtship and spawning (Hutter et al., 2011; Hutter et al., 2012). However, for human observers the differences in coloration between male and female zebrafish are often difficult to discriminate due to minor sexual dimorphism in body color in this species (Hutter et al., 2012). The conventional guidelines for sex determination in zebrafish by human perception include sex-related differences in various features such as color, shape, behavior and genital papilla (McMillan et al., 2015). For example, females often have a round shape and protruding abdomen compared to males, but not all females possess an obviously distended abdomen (Hutter et al., 2010; McMillan et al., 2015). Furthermore, sex determination using coloration in zebrafish is difficult because there is interindividual variation in color, and in some cases males and females exhibit similar body coloration (McMillan et al., 2015). However, a precise sex determination of adult fish can be performed using microscopic examination by dissecting gonad tissue, which requires killing animals 
(Abozaid et al., 2011), is labor-intensive and requires biological expertise. Hence, the current approaches for sex determination in zebrafish are subjective and not sufficiently reliable, and highly time-consuming (McMillan et al., 2015).

In recent years, machine learning has emerged as a promising technique for data processing in life science and computational biology (Angermueller et al., 2016; Liakos et al., 2018), particularly in clustering biological images and phenotypic classification (Grys et al., 2016; Jeanray et al., 2015). Deep learning models, a relatively new branch of machine learning, consist of multiple processing layers to learn representations of large data sets, and have dramatically advanced and improved the state-of-the-art in various research fields (LeCun et al., 2015; Min et al., 2017; Liakos et al., 2018). Convolutional neural networks (CNN), a deep learning architecture, recently surpassed human-level accuracy especially for image recognition and classification (Grys et al., 2016).

The main goal of this study was to develop an efficient technique for sex determination of zebrafish using two fully automatic machine learning methods: Deep Convolutional Neural Networks (DCNNs) based on body color and pattern, and Support Vector Machine (SVM) based on caudal fin color only. We first applied DCNNs to determine the sex from the image of the whole fish body. We secondly applied SVM using the color distribution of the caudal fin pictures to automatically determine the sex of zebrafish. Both approaches used RGB images as an input and were trained in a supervised manner to classify the sex of an individual using image analysis. The estimated color intensity based on SVM was then used to quantify the degree of association between coloration and body weight and length. To further demonstrate the utility of the approaches, the SVM technique was used to quantify the impact of high water temperature on color intensity of the caudal fins of different sexes. This technique can be used in various science areas, in which sexual plasticity and its ecological relevance will be the focus of scientific research.

\section{Materials and methods}

\section{Ethics statement}

All procedures in this study were in strict accordance with the German animal welfare act and national and international recommendations. This study was approved by the University of Goettingen committee for the care and use of animals (File number E3-17). The broodstocks were kept in the recirculation systems of aquaculture facilities according to the approved institutional guidelines on the use of animals for research purposes (Abozaid et al., 2011). 


\section{Experimental design and phenotypic measurements}

We designed a treatment-control study to examine the effect of high water temperature during the critical period of zebrafish embryogenesis on sex ratio, color intensity, body weight and length and their associations. For this, equal proportions of fertilized eggs from full-sib families of Singapore strain (Von Hertell et al., 1990; Hosseini et al., 2019b) were exposed to low $\left(28^{\circ} \mathrm{C}\right)$ and high temperatures $\left(35^{\circ} \mathrm{C}\right)$ during 5 to $24 \mathrm{hr}$ post-fertilization (hpf; Hosseini et al., 2019b). The standard rearing temperatures for zebrafish are between $26-29^{\circ} \mathrm{C}$ with an optimum of $28^{\circ} \mathrm{C}$ (Detrich et al., 2004; Ribas \& Piferrer, 2014). The high temperature applied in the temperature-exposed group of this study is in line with previously reported studies (Hosseini et al., 2019a; Hosseini et al., 2019b; Abozaid et al., 2011). The temperature of heatexposed groups was changed gradually in this experiment. Two weeks after hatching, the temperature treatment and control groups of different families were separately mixed in bigger tanks to eliminate the effect of population density within tanks and kept until sexual maturity (90 days post-fertilization). After maturation, total length and body weight of all individuals were measured, as described in Hosseini at al. (2019a). All individuals showed no caudal fin damage and exhibited normal morphology. The real sex in the control and temperature treatment groups was individually determined in adult fish by microscopic inspection. All husbandry facilities, fish management and water quality control, animal care and feeding, and data recording are described in detail by Hosseini et al. (2019a).

\section{Image acquisition}

For imaging of fish, each adult individual was removed from their tank and killed in ice water. The fish was then placed in a glass petri plate and photographed. The images of all adult fish were captured individually using a digital camera (Nikon D7200). The Nikon D7200 is an Advanced Photo System type-C (APS-C) digital single-lens reflex camera with a maximum image resolution of 6,000×4,000 (24 megapixels). This camera has an AF-S DX

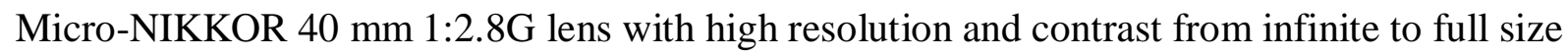
(1:1 magnification). For taking the pictures, the camera was fixed in a Kaiser RS3 Copy Stand (kaiser-fototechnik) at a distance of $17 \mathrm{~cm}$ from the object location, at which fish were placed in a glass petri plate. In order to provide the optimal conditions for eliminating specular reflections and shadow problems, two different lighting techniques were applied. First, a light panel; Rex-Slimline (Rex Leuchtplatten) was used for upward illumination and second, a Cold LED Light Source; KL 300 LED (SCHOTT AG Lighting and Imaging) was used for 
lateral illumination. All photographs were taken vertically from the lateral view of fish in an ordinary fish laboratory room under common fluorescent light and under a well-defined setting as described above. The same image recording procedure was used for all individuals in this study. All images were saved in RAW file and TIFF format with a resolution of $4,496 \times 3,000$ pixels. RAW files were then imported into the Photoshop software (Adobe Photoshop CS5, Photoshop Extended, version 12.0, 2010), for processing. The whole caudal fin region was cut out for SVM analysis. The caudal fin region was defined by the base of the fin at the caudal end of the body from the proximal to the distal end, which was clearly visible in the picture. We used one image of each of 448 animals to determine the sex. In an image preprocessing step, one out of 448 images was discarded from the dataset used for sex classification by machine learning due to low picture quality.

\section{Colour feature extraction}

Sex classification of the whole fish body and caudal fin was conducted using DCNNs and SVM. For the color-based classification of sex, color histograms (Swain \& Ballard, 1991) were used to train a SVM (Keerthi \& Lin, 2003). To do so, the images were first transformed from the RGB color space to the lab color space. Based on the converted lab image, histograms to describe the color distribution for each channel were calculated. For this purpose, all the colors in each image were counted and a frequency distribution generated. These histograms were used as features to train the SVM with a Gaussian kernel. A simple illustration of SVM with Gaussian kernel can be found in Figure 1. The main idea of training the SVM was to obtain a hyperplane that could separate the histograms in order to enable sex classification. The maps transform the histogram to a score value by calculating the distance from the histogram in a higher dimension to the hyperplane, giving a scalar value, which is given the L2 (Euclidian) distance (see materials and methods in Appendix S1). 


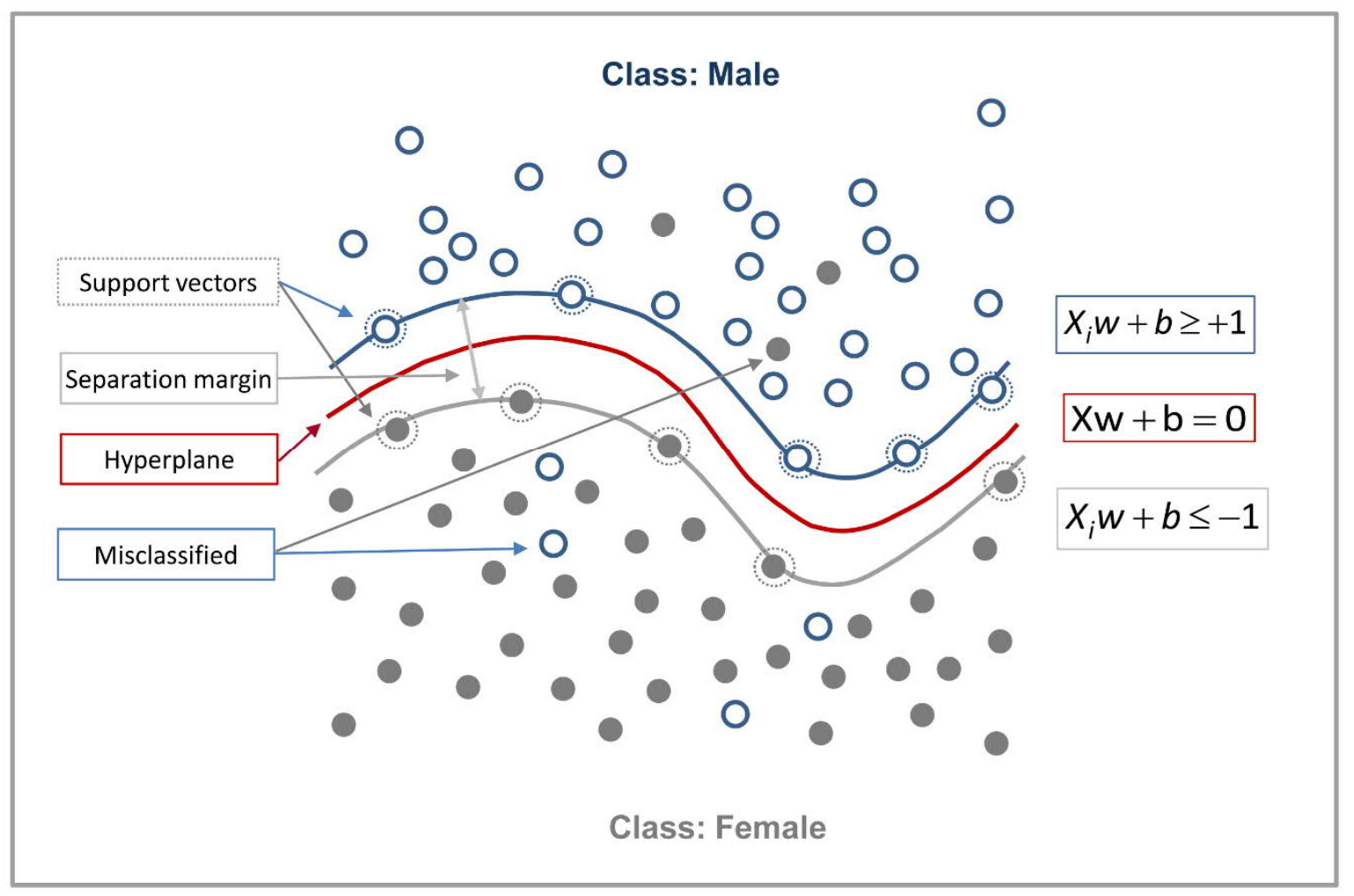

Figure 1: The schematic diagram represents the SVM classification with Gaussian kernel function used in this study for sex discrimination. $X_{i}$ presents samples (input data), w presents weights and $b$ illustrates the bias factor.

\section{Deep convolutional neural network}

A convolutional neural network is an artificial neural network introduced by Krizhevsky et al. (2012). Basically, the structure of a classical CNN consists of one or more convolutional layers, followed by a pooling layer. In principle, this unit can be repeated as often as required; with sufficient repetitions, this is referred to as DCNNs. The data processing through a network is done layer wise. The flow chart of the CNN architecture applied for sex classification in this study is illustrated in Figure 2. This CNN uses raw image pixels to model a simple differentiation score function. The architecture consists of the layers, which are arranged in 3 dimensions: width, height, and depth, where width and height are the dimensions of the image, and depth represents the color channels: red, green and blue. The original image is transformed layer by layer from the original pixel values to the final class score. As mentioned before, the INPUT layer receives the raw pixel values of an image as a 
matrix. In this case, an image is a tensor with the size $(255 \times 255 \times 3)$. The convolution layers calculate a point product from the comparatively small convolution matrix (also called filter kernel) with the currently underlying image section, whereby the convolution matrix moves stepwise over the input of the layer. For the first convolution layer, we used 96 convolution matrices (filters) of $3 \times 3$ pixels. In the next steps, the number of filters was increased to 384 . After normalization, the convolution input of each neuron was transformed by a Rectified Linear Unit (ReLU) activation function (Nair \& Hinton, 2010) into the output that models the relative significance. To prevent the vanishing of the gradient during training residual connections were used (He et al., 2015), where the outputs of one layer are re-added to the original input matrix. This process was repeated in a consecutive processing using the increased number of filters. To minimize overfitting, the last feature layer was reduced by a global mean pooling operation. At the end, the probabilities for a binary outcome, namely the sex, were determined. For this purpose, the Softmax function was used, which represents the probability distribution over different possible events (see materials and methods in Appendix $\mathrm{S} 1)$. 


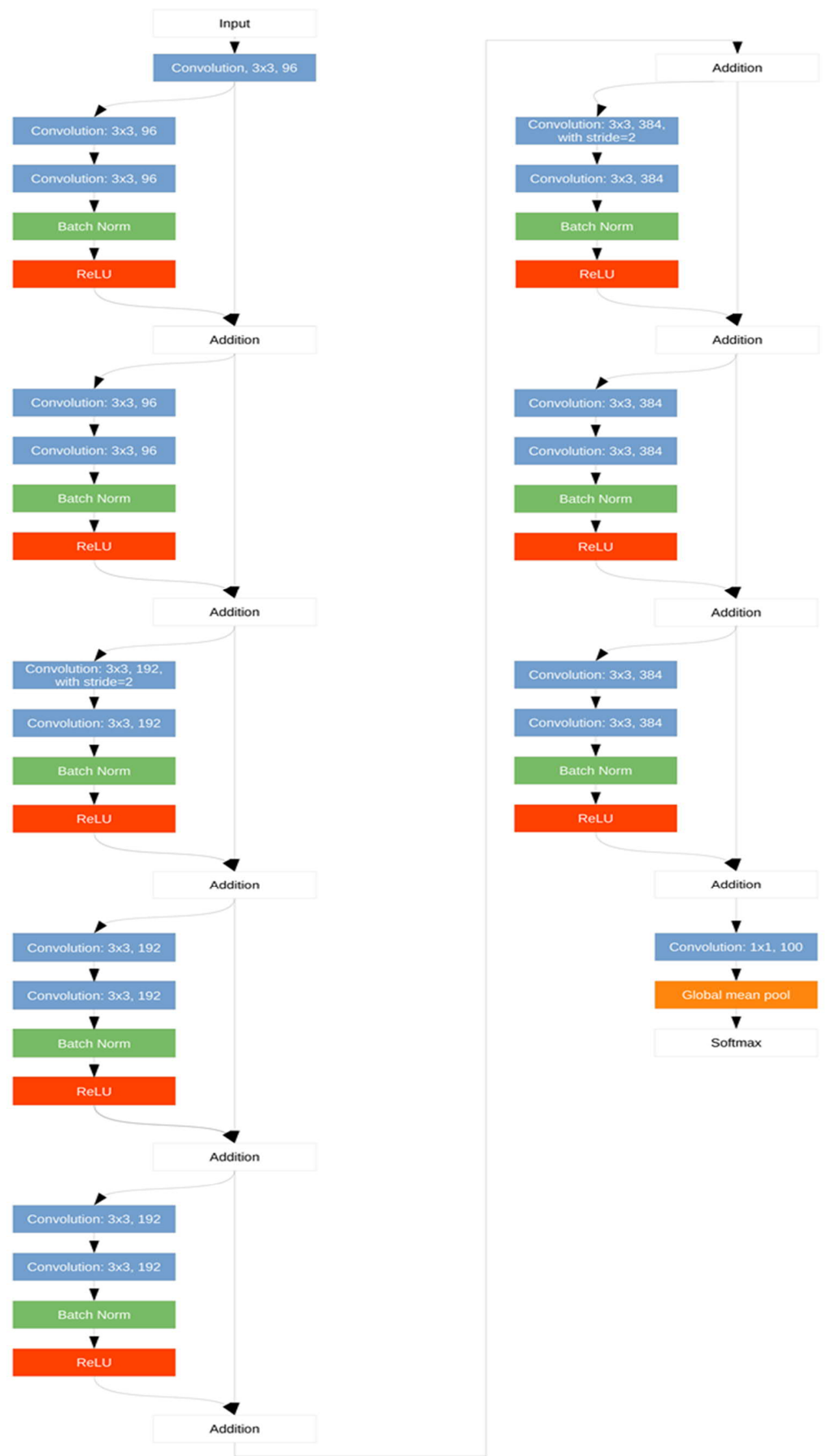

Figure 2: The graph represents the flow chart of the architecture of the convolutional neural networks (CNNs) applied for sex classification in this study. 


\section{Statistical analysis}

Statistical analysis of treatment effect on phenotypic sex of adult zebrafish was performed by applying a linear logistic model with a binary response variable, which was modelled as a Bernoulli random variable with $y_{i}$. The dependent variable $\left(y_{i}\right)$ can take the value 1 with the probability of being male $\pi_{\mathrm{i}}$ or 0 with the probability of being female $1-\pi_{\mathrm{i}}$ for observation $\mathrm{i}$.

The logistic model uses a link function $\mathrm{g}\left(\pi_{\mathrm{i}}\right)$ linking the expected value to the linear predictors $\eta_{i}$.

The data were then analyzed with the GLIMMIX procedure of SAS according to the following model:

$$
\operatorname{Logit}\left(\frac{\pi_{i}}{1-\pi_{i}}\right)=\eta_{i}=\mu+\alpha_{i}
$$

where $\pi_{\mathrm{i}}$ is the probability to be male, $\mu$ is the overall mean effect, $\alpha_{\mathrm{i}}$ is the fixed effect of temperature treatment $\left(\mathrm{i}=1\right.$ : temperature-treated eggs $35^{\circ} \mathrm{C}, \mathrm{i}=2$ : control group $\left.28{ }^{\circ} \mathrm{C}\right)$. Least squares means were estimated on the logit scale and then back-transformed to the probability scale using the inverse link function $\pi_{\mathrm{i}}=\exp \left(\eta_{\mathrm{i}}\right) /\left[1+\exp \left(\eta_{\mathrm{i}}\right)\right]$, applying the LSMEANS statement. Significant differences between least squares means were tested using a test procedure by inclusion of the PDIFF option in the LSMEANS statement and adjusted by Tukey-Kramer correction. Standard errors of least square means were calculated as described by Littell et al. (2006).

The impact of treatment and sex on body weight and length were analysed using the GLM procedure of SAS with the following model:

$\mathrm{y}_{\mathrm{ijk}}=\mu+\alpha_{\mathrm{i}}+\beta_{\mathrm{j}}+\alpha \beta_{\mathrm{ij}}+\varepsilon_{\mathrm{ijk}}$

where $y_{i j k}$ is the observation for body weight and length, $\mu$ is the general mean, $\alpha_{\mathrm{i}}$ is the effect of treatment (temperature treatment, control), $\beta_{\mathrm{j}}$ is the fixed effect of sex, $\alpha \beta_{\mathrm{ij}}$ is the fixed effect of interactions between treatment and sex and $\varepsilon_{\mathrm{ijk}}$ is the random error.

To determine the degree of association between the sex classifications using DCNNs and SVM with the real sex in a further analysis, the mean square contingency coefficient (phicoefficient; $\varphi$ ) was estimated using the FREQ procedure of SAS through the construction of contingency tables.

The SVM estimates are individual scores for being male or female based on RGB color, which were used for sex classification. The variation of the differences between these estimated scores for males and females reflects the degree of color intensity. An individual with a higher score for a certain sex, which is dependent on the color intensity, was classified 
as male or female. We created a new variable in the first step based on the differences between the estimated scores of males and females for each individual. Then, we computed the z-score of this variable by applying the z-transformation, which was used as a dependent variable in the statistical model in order to analyze the association between body weight and length and the degree of caudal fin pigmentation for the different sexes, treatments and their interactions as main factors. For this purpose, an analysis of covariance was applied using body weight and length as the covariate terms with up to 3 polynomial degrees, and considering the fixed main factors (real sex, treatment) and their interaction effects as well as the interactions between the main factors and the covariate (body weight or length) up to degree 3 of the polynomial. The final model was obtained by backward elimination of nonsignificant factors and factor combinations using F-statistics. Since extreme observations or outliers can influence the parameter estimations, outliers of the dataset were detected using the influence diagnostics recommended by Belsley et al. (1980). The influence diagnostics methods are incorporated into the REG and MIXED procedure of SAS by using the INFLUENCE option in the MODEL statement (SAS/STAT ${ }^{\circledR}$ 9.2User's Guide, the MIXED Procedure, 2008). The Studentized residuals are excellent statistics for detecting unusual observations. We estimated the Studentized residuals using the INFLUENCE option in the MODEL statement of SAS' mixed procedure to estimate residuals for each recorded data point. 20 records out of the 448 data points with absolute extreme values larger than 3 (Hosmer et al., 2013) were identified and discarded from subsequent statistical analysis. The final statistical model was applied with the mixed procedure of SAS as follows:

$\mathrm{y}_{\mathrm{ijk}}=\mu+\alpha_{\mathrm{i}}+\beta_{\mathrm{j}}+\alpha \beta_{\mathrm{ij}}+\mathrm{b}_{1}\left(\mathrm{x}_{\mathrm{ij}}\right)+\mathrm{b}_{2} \beta_{\mathrm{j}}\left(\mathrm{x}_{\mathrm{ij}}\right)+\varepsilon_{\mathrm{ijk}}$

where $y_{\mathrm{ijk}}$ is the quantile of the standard normal distribution (z-score) as described above, $\mu$ is the general mean, $\alpha_{\mathrm{i}}$ is the effect of treatment (temperature treatment, control), $\beta_{\mathrm{j}}$ is the fixed effect of sex, $\alpha \beta_{\mathrm{ij}}$ is the fixed effect of an interaction between treatment and sex, $b_{1}$ is the linear regression coefficient of body weight or length $(\mathrm{x}), \mathrm{b}_{2}$ is the linear regression coefficient of interaction between sex and body weight or length (x) and $\varepsilon_{\mathrm{ijk}}$ is the random error. SAS system version 9.3 (SAS Institute Inc. 2014) was used for all aforementioned statistical analyses. 


\section{Results}

\section{Sex classification detected via machine learning methods}

For evaluating the classification performance of the two machine learning methods, the result of these methods were compared with the real sex output in the different experimental groups. The descriptive statistical analysis of the real sex using microscopic inspection of gonadal tissues showed that high temperature resulted in an increase in the proportion of males compared to the control group $(79.80 \%$ vs. $50.20 \%)$. These differences were highly significant by applying generalized linear model $(\mathrm{p}<0.0001)$. The detailed results of this part of the experiment are given in Hosseini et al. (2019b). According to the DCNNs analysis the percentage of male was $79.80 \%$ in the heat-treated group versus $51.41 \%$ in the control group. The descriptive statistic for SVM showed $67.17 \%$ male in the treatment compared to $49.40 \%$ in the control group. The result of the generalized linear model analysis revealed that these differences were also highly significant for both machine learning approaches. Classification of sex using DCNNs and SVM methods and the degree of association with the real sex are presented in Figure 3. The DCNNs were trained to classify the sex based on color and pattern of fish pictures, while SVM classified the sex based on the caudal fin color only. Our analyses demonstrated a high agreement $(\varphi=0.97)$ between the sex ratio determined by DCNNs and the real sex ratio in the control group. The same applies to sex determination using SVM in the control group, which showed a slightly lower association with the real sex $(\varphi=0.96$; Figure 3a,b). In temperature-treated animals, DCNNs were able to determine the sex in complete agreement with the real sex $(\varphi=1.0)$, whereas sex classification by color features in the caudal fin using SVM showed a lower association with the real sex $(\varphi=0.71$; Figure $3 c, d)$. In this analysis, 25 animals were misclassified as females using SVM, all of which were temperature-treated male animals. Inspection of the pictures of those animals showed a lower caudal fin pigmentation intensity compared to the regular males in the control group. To investigate this further, we performed a new analysis considering the color intensity of these animals resulting from SVM as a new level of the main factor sex and compared them with the color intensity score of correctly classified males in the temperature-treated group, which resulted in a significant difference in color intensity between these two groups (-0.3526 vs. 0.6004; $\mathrm{p}<0.0001)$. We suppose these animals were neomales who had altered their sex from genotypic females to phenotypic males in response to high water temperature and showed lower pigmentation intensity due to the temperature-induced masculinization. 


\section{Female (real sex)}

(a) $\quad \varphi=0.97$

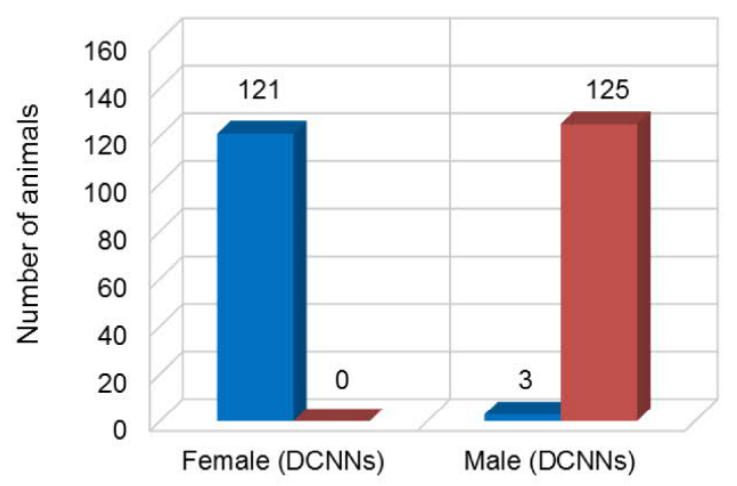

(b) $\varphi=0.96$
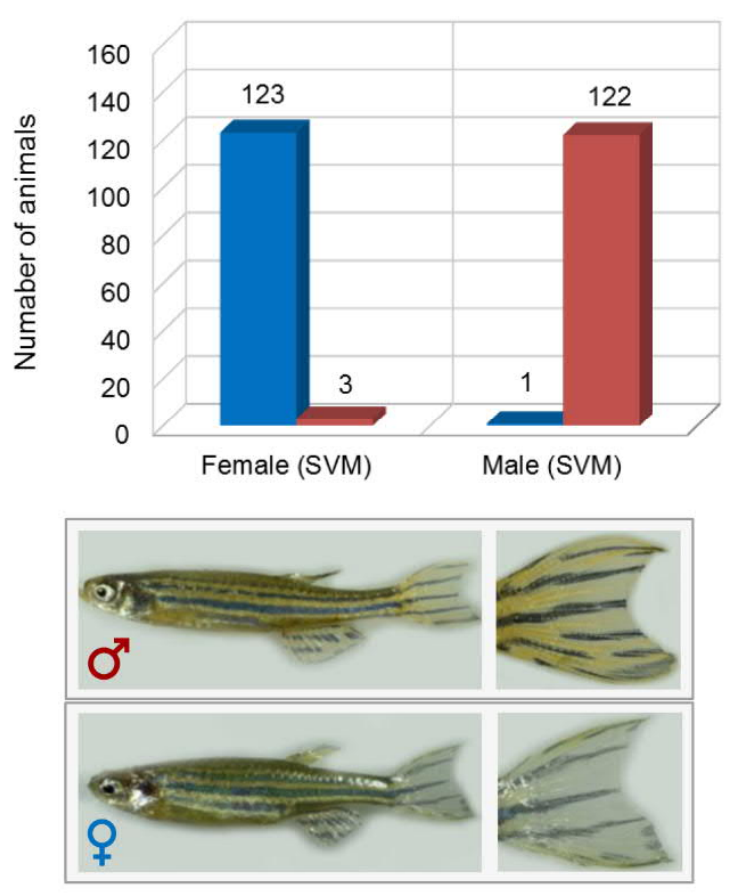

Control (c) $\varphi=1.0$

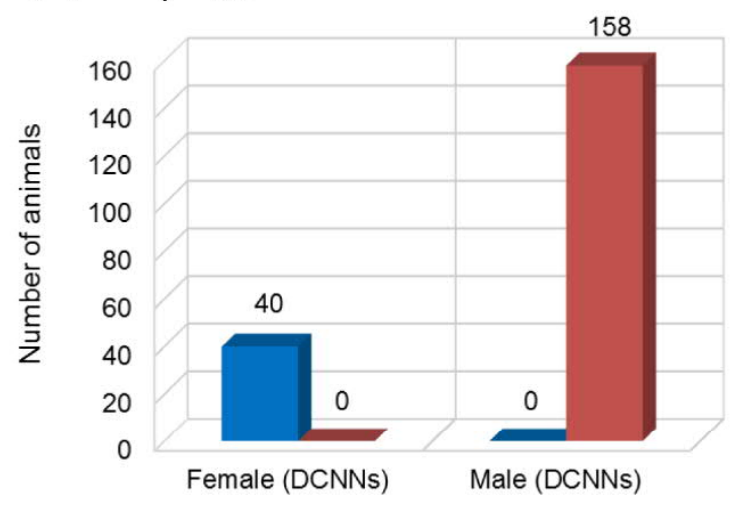

(d) $\varphi=0.71$
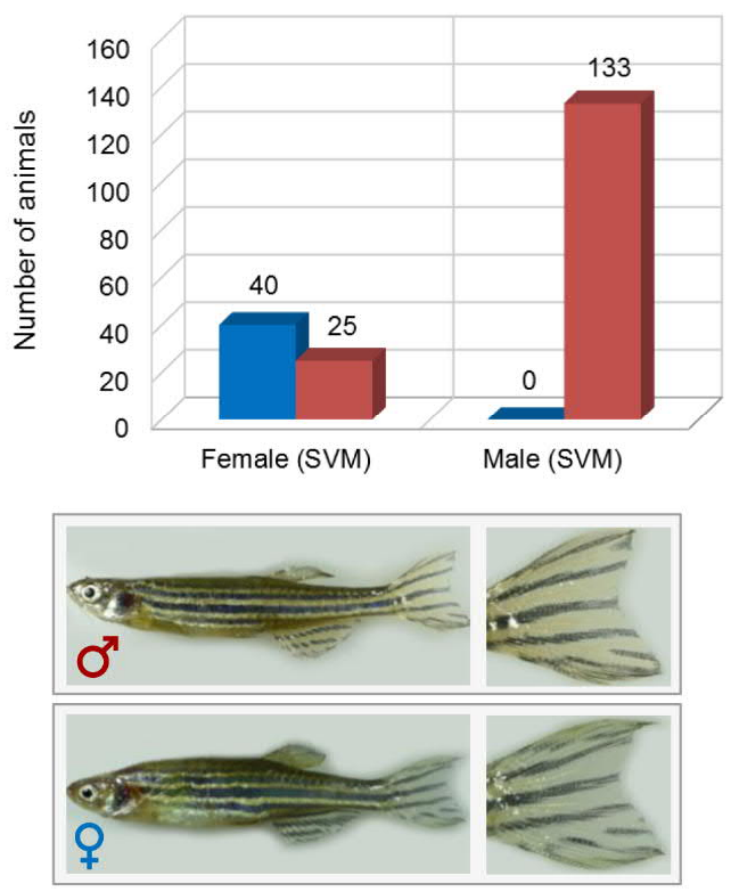

Treatment

Figure 3: The result of sex classification using deep convolutional neural networks (DCNNs) and support vector machine (SVM) methods compared with real sex. The degree of agreement $(\varphi)$ between sex classification using DCNNs analysis of adult fish body features and SVM analysis using color of caudal fin with real sex in control $(a, b)$ and temperature treatment groups $(c, d)$. 


\section{Association between color pattern intensity and zootechnical parameters}

In a further stage of this study, the degree of caudal fin coloration in association with body weight and length for the fixed effects of sex, treatment and their interactions were analysed (Figure 4 and 5). As expected, the sex as a main factor in the statistical model leads to distinct differences in pigmentation intensity between males and females without considering any covariates (body weight or length) in the statistical model. Males showed a higher pigmentation intensity compared to females (male: 0.768 vs. female: -1.165 ). Considering the main factor treatment, the treated animals exhibited a lower pigmentation intensity compared to the control group (treatment: -0.0869 vs. control: -0.3102 ), in response to high water temperature. We found a significant sex-temperature interaction on pigmentation intensity. Comparing the pigmentation intensity of females without considering any covariates in the control and treatment groups revealed that there is no significant difference in coloration between these two groups. In contrast to this, high temperature treatment resulted in a distinct reduction in color intensity of males, which partly contributed to a significant treatment effect (Figure 4a). This result indicates that temperature treatment during early embryogenesis might have an impact on the expression of pigmentation genes responsible for development of the caudal fin coloration and phenotypic variation in males. The same is true considering the adjusted means derived from the main factors and interactions using body weight or length as covariates (Figure $4 \mathrm{~b}, \mathrm{c}$ ). The result of the analysis of covariance also showed a significant positive association between body weight and length of adult fish with the degree of caudal fin color intensity in males both in the control and heat-induced groups, whereas no significant association was detected in females (Figure 5a,b). To the best of our knowledge, this finding has not been reported in zebrafish so far. 


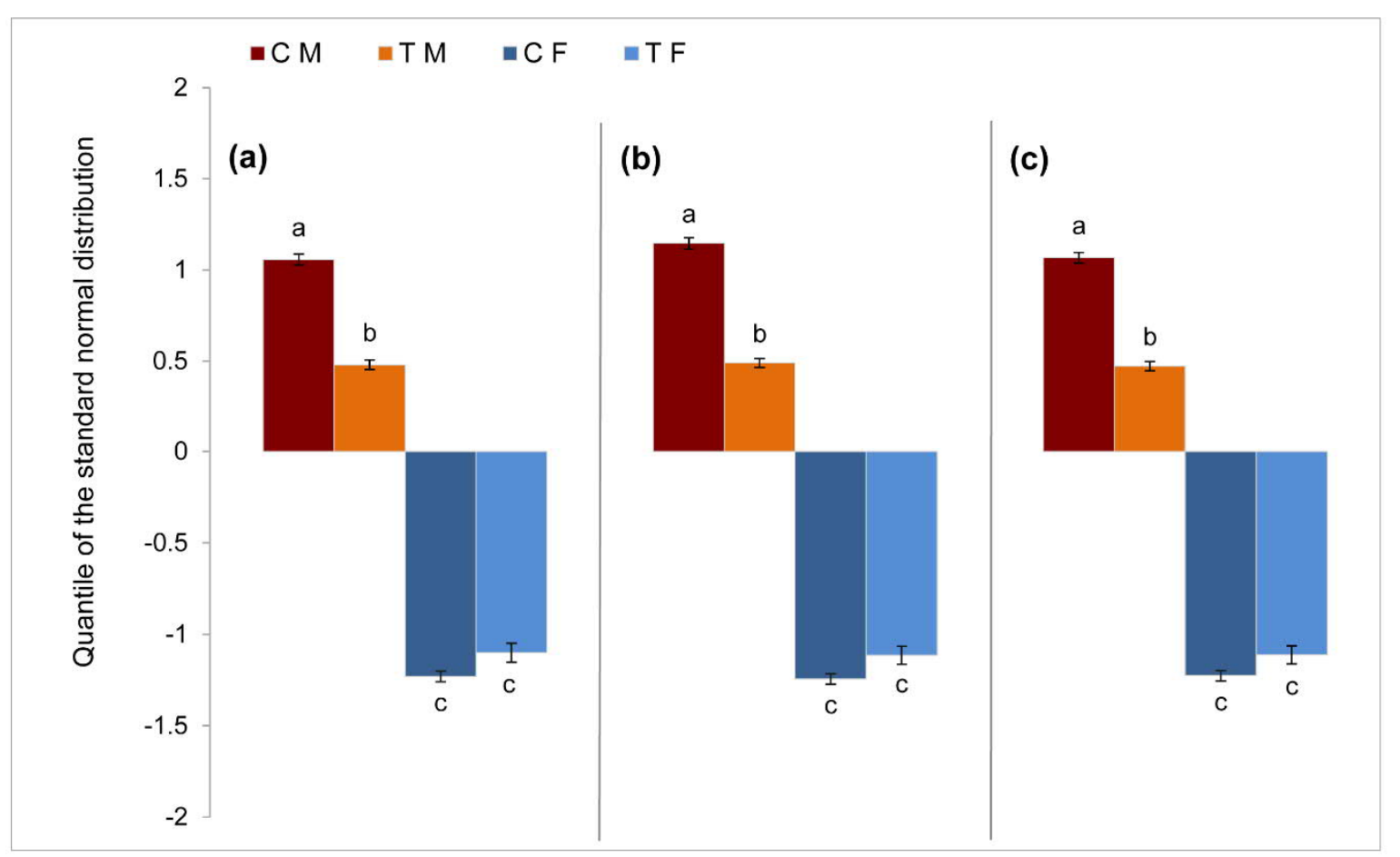

Figure 4: Association between degree of caudal fin coloration using SVM with body weight and length in different experimental groups: CF (control female), CM (control male), TF (treatment female) and TM (treatment male). (a) LS-Means for the levels of Treatment $\times$ Sex interaction without considering any covariates in statistical model and considering the covariate of body weight (b) and total length (c). Different alphabets (a-c) illustrate the significant differences between the least squares means of different factor levels $(\mathrm{P}<0.0001)$. $\mathrm{Y}$ axis represents the color intensity of caudal fins derived from SVM. 


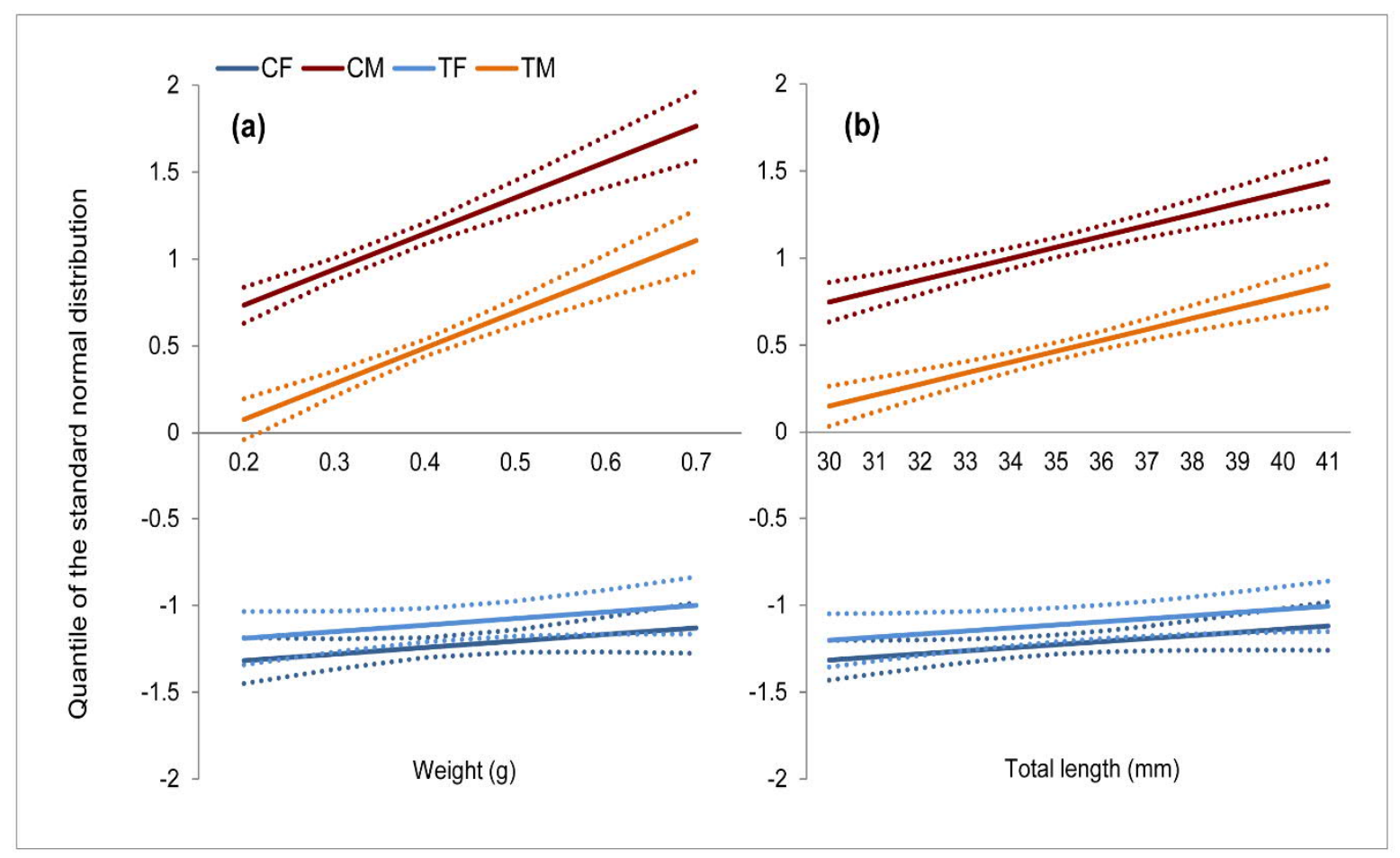

Figure 5: The effect of body weight (a) and total length (b) on pigmentation intensity in different experimental groups: CF (control female), CM (control male), TF (treatment female) and TM (treatment male). The solid lines show the LS-means at certain level of body weight and total length. The dash bars present the confidence limits of LS-means. Y axis represents the color intensity of caudal fins derived from SVM.

\section{Body weight and length}

Table 1 summarizes the least squares means and the significance of explanatory variables on body weight and total length in adult zebrafish. The main factor treatment and sex as well as their interactions (treatment $\times$ sex) showed a significant influence on body weight. However, only a significant influence of the main factor sex on length was observed. Zebrafish raised at a high temperature (HT) were heavier (0.4161 vs. 0.3952) and longer (35.4976 vs. 34.8982) than those raised at a low temperature (LT) condition. In the case of sex, a significant difference of body weight was observed between males and females; a considerably higher body weight was found in the female group versus males, while the effect on total length was not significant. The differences in body weight between LT- and HT females for the effect of 
interaction between treatment $\times$ sex were not significant. However, temperature treatment resulted in a significant reduction of body weight in males.

Table 1: Least squares means, standard error $( \pm \mathrm{SE})$ and ANOVA significance level for body weight $(\mathrm{g})$ and total length $(\mathrm{mm})$ in adult zebrafish for the effect of temperature, sex and treatment $\times$ sex interaction

\begin{tabular}{|c|c|c|c|}
\hline \multirow{2}{*}{ Effect } & \multicolumn{3}{|c|}{ Traits } \\
\hline & & Body weight & Total length \\
\hline \multicolumn{4}{|l|}{ Treatment } \\
\hline Low temperature ${ }^{1}(\mathrm{LT})$ & & $0.3952 \pm 0.0049^{\mathrm{a}}$ & $34.8982 \pm 0.1323^{\mathrm{a}}$ \\
\hline High temperature ${ }^{2}(\mathrm{HT})$ & & $0.4161 \pm 0.0067^{\mathrm{b}}$ & $35.4976 \pm 0.1816^{\mathrm{b}}$ \\
\hline \multicolumn{4}{|l|}{ Sex } \\
\hline Male & & $0.3773 \pm 0.0047^{\mathrm{a}}$ & $35.1049 \pm 0.1253^{\mathrm{a}}$ \\
\hline Female & & $0.4339 \pm 0.0069^{b}$ & $35.2909 \pm 0.1865^{\mathrm{a}}$ \\
\hline \multicolumn{4}{|l|}{ Treatment $\times$ Sex } \\
\hline LT $\times$ Male & & $0.3582 \pm 0.0070^{\mathrm{a}}$ & $34.9648 \pm 0.1882^{\mathrm{a}}$ \\
\hline HT $\times$ Male & & $0.3965 \pm 0.0062^{b}$ & $35.2450 \pm 0.1653^{\mathrm{a}}$ \\
\hline $\mathrm{LT} \times$ Female & & $0.4321 \pm 0.0069^{b}$ & $34.8316 \pm 0.1859^{a}$ \\
\hline HT $\times$ Female & & $0.4357 \pm 0.0121^{b}$ & $35.7502 \pm 0.3233^{\mathrm{a}}$ \\
\hline $\begin{array}{l}\text { ANOVA significance } \\
\text { level }[\mathrm{P}(\mathrm{F})]\end{array}$ & Treatment & Sex & Treatment $\times$ Sex \\
\hline Body weight & 0.0131 & $<0.0001$ & 0.0392 \\
\hline Total length & 0.0079 & 0.4082 & 0.1560 \\
\hline
\end{tabular}

Note: Different alphabets (a-c) illustrate the significant differences between the least squares means of different factor levels $(\mathrm{P}<0.05)$.

${ }^{1}$ Low temperature refers to the control group at $28^{\circ} \mathrm{C}$.

${ }^{2} \mathrm{High}$ temperature refers to the temperature treatment group at $35^{\circ} \mathrm{C}$. 


\section{Discussion}

Machine learning approaches recently became the leading technique for object and action recognition in humans (Toshev et al., 2013; Jain et al., 2013). The technique has great potential in animal sciences for studying of different aspects of animal behavior such as movement, food intake, social structure and competition, reproduction behaviour, communication and welfare and nesting using complex datasets (Stern et al., 2015; XU \& Cheng, 2017; Borchers et al., 2017; Valletta et al., 2017; Wang, 2019). Automated imaging technologies provide a large number of images that require an efficient strategy of analysis such as machine learning. Thus, image analyses can be used to identify and classify objects in various biological research aspects (Grys et al., 2016; Liakos et al., 2018). The study of Jeanray et al. (2015) is an example of image analysis for classifying phenotypical deformity in zebrafish larvae using machine learning methods. Their study on phenotypic classification of images resulted in a high agreement with manual classification by biological experts. Automation of analysis and classification of sex using machine learning methods directly from images is a great possibility to increase the efficiency in the prediction process (Singh \& Goel, 2016). The current guidelines for phenotypic sex classification in zebrafish using conventional methods are subjective and rely on different phenotypic observations by humans, which are highly labor-intensive and potentially prone to error in the prediction process (McMillan et al., 2015). For example, zebrafish quickly become pale after removing from the water (Hutter et al., 2010), which may refer to the presence or absence of iridophore pigment cell types. Iridophores give the body a shiny appearance by reflecting light in the water (Patterson \& Parichy, 2013; Frohnhöfer et al., 2013). Iridophores are present in the body coloration, but are not involved in the fin color formation (Singh \& Nüsslein-Volhard, 2015). It is often difficult to distinguish between sexes using different body colors outside of water. In this study, we presented two fully automated machine learning methods (DCNNs and SVM) as efficient, robust, and flexible approaches for sex classification in zebrafish using a set of images for the first time in a color space designed for human perception. Machine learning classification relies on the quality, size and objectivity of learning datasets, which is more reliable than manual approaches for sex determination in zebrafish. Our method is not specific to zebrafish; it is a general approach that could be applicable to other organisms for future research. Based on phenotypic characteristics in this study, a high accuracy of sex differentiation was obtained using these two methods in nonheat-treated groups.

Furthermore, in this study, we reported a less intense color in the caudal fin of a subset of adult zebrafish using SVM who were exposed to elevated water temperature during 
embryogenesis. Based on this result, classification of sex using SVM technique is applicable for sexing in nonexperimentally manipulated individuals, but it may be less effective when the phenotypes (color) are experimentally altered. Therefore, some treated males in this study were misclassified using SVM due to reduced pigmentation intensity. However, DCNNs was able to classify the sex with high performance independent of the alteration of phenotypes.

The effect of high temperature on loss of pigmentation has already been observed in adult zebrafish in another study, where the animals were thermally influenced during the larval stage (Ribas et al., 2017). Since in our study the pigmentation deficiency was mainly observed in heat-treated males, suggesting that these animals might have been sex-reversed or masculinized females. Therefore, it was hypothesized that decreased color intensity in masculinized animals would potentially influence sexual attractiveness, and thus, mating success. The molecular genetic study of heat-induced masculinization in European sea bass showed that masculinization process is caused by epigenetic modifications (Navarro-Martin et al., 2011). In a recent study, transcriptome analysis was used to distinguish neomales from the normal male zebrafish (Ribas et al., 2017). Hence, genetic and epigenetic investigations can be used to discriminate the masculinized from normal animals and to elucidate the physiological mechanisms of sexual-reversed males in interaction with environment. However, the underlying mechanism of color intensity in interaction with the environmental temperature is still not clear and deserves further research.

Sexual selection represents a mode of selection on certain traits to increase an individual's reproductive success. Regarding sexual selection theory, males of many species develop a variety of secondary sexual characters and traits, such as body ornaments and pronounced coloration, to signal their attractiveness as mating partners (Uusi-Heikkilä et al., 2012). In zebrafish, females usually have a rounder shape than males, and males display a more intense yellow coloration than females particularly during sexual activity (Gerlai et al., 2000, Hutter et al., 2010; Singh \& Nüsslein-Volhard, 2015), which appears to be important for sexual attraction. Since reproduction is energetically costly it is generally expected that there is a positive relationship between partner quality and reproductive investment in terms of differential allocation theory (Uusi-Heikkilä et al., 2012). In a study on a laboratory strain of zebrafish, neither female preferences nor spawning successes were associated with the male body size (Spence \& Smith, 2006). Furthermore, the effect of male body size for female preferences could not be detected because the hypothesis of the study was focused on differences in male dominance, not on male body size with a large size variation (Spence $\&$ Smith, 2006). In contrast, other studies demonstrated a clear female preference for larger 
males (Pyron, 2003; Skinner \& Watt, 2007; Uusi-Heikkilä et al., 2012). In these studies, females allocate their reproductive resources to larger males, characterized by a higher spawning probability and clutch size. In our study using machine learning techniques, we found a pronounced secondary sexual characteristic (color) in zebrafish males compared to females. In addition, a positive association between color intensity and body weight and length was observed in male zebrafish. Our results in this study suggest that male coloration and its positive association with body weight and length may play a role in sexual attraction in the zebrafish strain studied here and may increase reproductive success. However, whether caudal fin coloration and its interaction with body size serves as a sexually selected trait for mating success in this species requires further research. Sexual preferences using coloration and size have been observed in other fish species. For example, mating preference in C. cyanea is related to male body size and orange caudal fin color intensity (Gronell, 1989; Wacker et al., 2016) and in G. aculeatus to redness of the throat in males (Kraak et al., 1999). Another aspect of this study was to investigate the impact of elevated water temperatures on growth performance. The high temperatures during embryonic development positively influenced both traits of growth, namely body weight and length. Schnurr et al. (2014) also observed a higher growth rate in zebrafish treated with high temperature during embryonic development compared to the control group. Our previous study confirms this too; a positive effect of increased temperature during embryogenesis on growth rate of another zebrafish strain was observed, which was more pronounced in females than in males (Hosseini et al., 2019a). Embryonic temperature in zebrafish affects thermal acclimation of muscle tissue, which has an influence on energy metabolism and swimming performance in adult fish, due to differences in the expression of genes involved in energy metabolism, cell stress, muscle contraction, and apoptosis (Scott \& Johnston, 2012). Effects of temperature during embryonic development on growth rate were also reported in other fish species such as gilthead sea bream. Exposure to different temperature (low temperature: $18^{\circ} \mathrm{C}$, high temperature: $22^{\circ} \mathrm{C}$ ) during embryogenesis in sea bream and its effect on muscle growth rate and body weight revealed that early temperature treatment has an influence on the expression profiles of a part of muscle developmental genes (Hsp90a, UNC45, MyoD and IGF1) and their expression is influenced by different temperature treatment (Serrana et al., 2012). In our study, we found a positive effect of increased temperature on growth performance in adult fish in response to high ambient temperature, which may partly be due to the effect of temperature on muscle developmental gene expression in zebrafish. 
Generally, developmental plasticity can be influenced by many different environmental factors, which induce the expression of different phenotypes. A classic example of plastic responses to environment is the effect of temperature on different phenotypic traits such as sex determination, body size, pigmentation, and survival in many animal species (Lafuente \& Beldade, 2019). Some plastic characteristics are simultaneously influenced by the same environmental factor and change in combined form in response to the environment. Some of such plastic traits might be adaptive, while some others might be maladaptive. These plastic responses to the environmental cues lead to phenotypic variation and diversification in a population, which affects response to selection (Lafuente \& Beldade, 2019). However, phenotypic expression may be unfavourable as a result of plasticity under certain environmental conditions and only a subpopulation with certain phenotypic expression may adapt. Thus, the survival of an adapted subpopulation only could lead to an impoverishment of genetic variation, or in general might have unfavourable effects and the species could be threatened with extinction (Burger \& Lynch, 1995; Brown et al., 2015). As shown in this study, the correlated plastic responses of phenotypic traits, including sex determination and color to high temperatures might contribute to population dynamics and a risk of extinction in changing environments (Bürger \& Lynch, 1995; Allendorf \& Luikart, 2007), particularly under extreme and fluctuating environmental conditions, which is relevant from an ecological point of view.

\section{Conclusions}

Phenotypic classification of sex using machine learning methods in this study resulted in a high degree of association with the real sex, which possesses the advantage of automatisation and robustness with a high degree of accuracy compared to conventional methods. In addition, we found that high ambient temperature leads to a lower color intensity in some treated males, which was quantified by SVM in the caudal fin, suggesting that these animals were likely masculinized animals or neomales. Our findings further indicated that the color intensity is associated with body weight and length in males but not in females, which appears to play a significant role in sexual attractiveness. Furthermore, none of our techniques and developed software in this study is specific to zebrafish; the same approach can be readily applied to other species with phenotypic sexual dimorphism. 


\section{Acknowledgments}

The authors are grateful to the Aquaculture and Aquatic Ecology staff of the University of Goettingen for providing the use of aquaculture facilities and animals.

\section{Conflict of interest}

The authors declare that they have no conflict of interest.

\section{Author contributions}

SHo contributed to the conception and design of the study, carried out the experiments, interpreted the results and wrote the manuscript. ARS substantially designed the study and conception. ARS and SHo performed computational data analysis and interpreted the data. SHe performed image analysis using DCNNs and SVM methods. ARS, HS, SHe, BB and JT edited and corrected the manuscript. All authors read and commented on the manuscript and approved the final version.

\section{Data accessibility statement}

The datasets used and analysed in this study are included in the article and are available in Supporting Information (DataS1-S2). Uses of dataset are in permission of corresponding author.

\section{Developed software}

The software for sex classification in this study is freely available in https://hdl.handle.net/21.11101/0000-0007-D837-8.

\section{References}

Abozaid, H., Wessels, S. \& Hörstgen-Schwark, G. (2011). Effect of rearing temperatures during embryonic development on the phenotypic sex in zebrafish (Danio rerio). Sexual Development, 5, 259-265. https://doi: 10.1159/000330120

Allendorf, F. W., \& Luikart, G. (2007). Conservation and the genetics of populations. Blackwell Publishing, Oxford, UK, ISBN-13: 978-1-4051-2145-3. 
Angermueller, C., Pärnamaa, T., Parts, L., \& Stegle, O. (2016). Deep learning for computational biology. Molecular Systems Biology, 12, 878. https://doi: $10.15252 / \mathrm{msb} .20156651$

Belsley, D. A., E. Kuh, and R. E. Welsch. (1980). Regression diagnostics: Identifying influential data and sources of collinearity. Wiley, New York, NY.

Borchers, M. R., Chang, Y. M., Proudfoot, K. L., Wadsworth, B. A., Stone, A. E., \& Bewley, J. M. (2017). Machine-learning-based calving prediction from activity, lying, and ruminating behaviors in dairy cattle. Journal of Dairy Science, 100, 5664-5674. https://doi: 10.3168/jds.2016-11526

Brown, A. R., Owen, S. F., Peters, J., Zhang, Y., Soffker, M., Paull, G. C., Hosken, D. J., Wahab, M. A., \& Tyler, C. R. (2015). Climate change and pollution speed declines in zebrafish populations. Proceedings of the National Academy of Sciences of the United States of America, 112, E1237-E1246. https://doi: 10.1073/pnas.1416269112

Bürger, R., \& Lynch, M. (1995). Evolution and extinction in a changing environment: A quantitative-genetic analysis. Evolution, 49, 151-163.

Detrich, H. W., Zon, I. L., \& Westerfield, M. (2004). The Zebrafish: Genetics, Genomics and Informatics, 2nd edn. Academic Press, San Diego.

Frohnhöfer, H. G., Krauss, J., Maischein, H. M., \& Nüsslein-Volhard, C. (2013). Iridophores and their interactions with other chromatophores are required for stripe formation in zebrafish. Development, 140, 2997-3007. https://doi: 10.1242/dev.096719

Gerlai, R., Lahav, M., Guo, S. \& Rosenthal, A. (2000). Drinks like a fish: zebra fish (Danio rerio) as a behaviour genetic model to study alcohol effects. Pharmacology Biochemistry and Behavior, 67, 773-782. https://doi.org/10.1016/S00913057(00)00422-6

Gronell, A. M. (1989). Visiting behaviour by females of the sexually dichromatic damselfish, Chrysiptera cyanea (Teleostei: Pomacentridae): a probable method of assessing male quality. Ethology, 81, 89-122. https://doi.org/10.1111/j.1439-0310.1989.tb00760.x

Grys, B. T., Lo, D. S., Sahin, N., Kraus, O. Z., Morris, Q., Boone, C., \& Andrews, B. J. (2016). Machine learning and computer vision approaches for phenotypic profiling. Journal of Cell Biology, 216, 65-71. https://doi: 10.1083/jcb.201610026

He, K., Zhang, X., Ren, S., \& Sun, J. (2015). Deep Residual Learning for Image Recognition. Proceedings of the IEEE conference on computer vision and pattern recognition, 770778.

Hosmer, D. W., Lemeshow, S., \& Sturdivant, R. X. (2013). Applied Logistic Regression. Wiley Series in Probability and Statistics. Print ISBN:9780470582473 
Hosseini, S., Brenig, B., Tetens, J., \& Sharifi, A. R. (2019a). Phenotypic plasticity induced using high ambient temperature during embryogenesis in domesticated zebrafish, Danio rerio. Reproduction in Domestic Animals, https://doi: 10.1111/rda.13382

Hosseini, S., Ha, N. T., Simianer, H., Falker-Gieske, C., Brenig, B., Franke, A., HörstgenSchwark, G., Tetens, J., Herzog, S., \& Sharifi, A. R. (2019b). Genetic mechanism underlying sexual plasticity and its association with colour patterning in zebrafish (Danio rerio). BMC Genomics, https://doi: 10.1186/s12864-019-5722-1

Hutter, S., Hettyey, A., Penn, D. J., \& Zala, S. M. (2012). Ephemeral Sexual Dichromatism in Zebrafish (Danio rerio). Journal of Ethology, 118, 1208-1218. https://doi: 10.1111/eth.12027

Hutter, S., Penn, D. J., S. Magee, S. \& Zala, S. M. (2010). Reproductive behaviour of wild zebrafish (Danio rerio) in large tanks. Behaviour, 147, 641-660. https://doi:10.1163/000579510X12632972473944

Hutter, S., Zala, S. M., \& Penn, D. J. (2011). Sex recognition in zebrafish (Danio rerio). Journal of Ethology, 29, 55-61. https://doi: 10.1007/s10164-010-0221-5

Jain, A., Tompson, J., Andriluka, M., Taylor, G. W. \& Bregler, C. (2013). Learning Human Pose Estimation Features with Convolutional Networks. arXiv preprint arXiv:1312.7302

Jeanray, N., Marée. R., Pruvot, B., Stern, O., Geurts, P., Wehenkel, L., \& Muller, M. (2015). Phenotype classification of zebrafish embryos by supervised learning. PLoS ONE, 10, e0116989. https://doi: 10.1371/journal.pone.0116989

Keerthi, S. S., \& Lin, C. J. (2003). Asymptotic Behaviors of Support Vector Machines with Gaussian Kernel. Neural computation, 15, 1667-1689.

Kobayashi, Y., Nagahama, Y., \& Nakamura, M. (2013). Diversity and plasticity of sex determination and differentiation in fishes. Sexual Development, 7, 115-125.

Kraak, S. B. M., Bakker, T. C. M., \& Mundwiler, B. (1999). Sexual selection in sticklebacks in the field: correlates of reproductive, mating, and paternal success. Behavioral Ecology, 10, 696-706. https://doi.org/10.1093/beheco/10.6.696

Krizhevsky, A., Sutskever, I., \& Hinton, G. E. (2012). ImageNet classification with deep convolutional neural networks. In Advances in neural information processing systems, pp. 1097-1105.

Lafuente, E., \& Beldade, P. (2019). Genomics of Developmental Plasticity in Animals. Frontiers in Genetics, 10, 720. https://doi: 10.3389/fgene.2019.00720

LeCun, Y., Bengio, Y., \& Hinton, G. (2015). Deep learning. Nature, 521, 436-44. https://doi: 10.1038/nature14539 
Liakos, K. G., Busato, P., Moshou, D., Pearson, S., \& Bochtis, D. (2018). Machine Learning in Agriculture: A Review. Sensors (Basel), 18, pii: E2674. https://doi: $10.3390 / \mathrm{s} 18082674$

Littell, R. C., Milliken, G. A., Stroup, W. W., \& Wolfinger, R. D. (2006). SAS system for Mixed Models. (pp. 633). Cary, NC: SAS Institute Inc.

McMillan, S. C., Géraudie, J., \& Akimenko, M. A. (2015). Pectoral fin breeding tubercle clusters: a method to determine zebrafish sex. Zebrafish, 12, 121-3. https://doi: 10.1089/zeb.2014.1060

Min, S., Lee, B., \& Yoon, S. (2017). Deep learning in bioinformatics. Briefings in Bioinformatics, 18, 851-869. https://doi: 10.1093/bib/bbw068

Nair, V., \& Hinton, G. E. (2010). Rectified Linear Units Improve Restricted Boltzmann Machines. Proceedings of the 27 th International Conference on Machine Learning.

Navarro-Martin, L., Vinas, J., Ribas, L., Diaz, N., Gutierrez, A., Di Croce, L., \& Francesc, P. (2011). DNA Methylation of the Gonadal Aromatase (cyp19a) Promoter Is Involved in Temperature-Dependent Sex Ratio Shifts in the European Sea Bass. PLoS Genetics, 7, e1002447. https://doi:10.1371/journal.pgen.1002447

Nowik, N., Podlasz, P., Jakimiuk, A., Kasica, N., Sienkiewicz, W., \& Kaleczyc, J. (2015). Zebrafish: an animal model for research in veterinary medicine. Polish Journal of Veterinary Sciences, 18, 663-74. doi: 10.1515/pjvs-2015-0086

Nüsslein-Volhard, C., \& Singh, A. P. (2017). How fish color their skin: A paradigm for development and evolution of adult patterns. BioEssays, 39. https://doi: 10.1002/bies.201600231

Patterson, L. B., \& Parichy, D. M. (2013) Interactions with iridophores and the tissue environment required for patterning melanophores and xanthophores during zebrafish adult pigment stripe formation. PLoS Genetics, 9, e1003561. https://doi: 10.1371/journal.pgen.1003561

Price, A. C., Weadick, C. J., Shim, J., Rodd, F. H. (2008). Pigments, patterns, and fish behavior. Zebrafish, 5, 297-307. https://doi: 10.1089/zeb.2008.0551

Pyron, M. (2003). Female preferences and male-male interactions in zebrafish (Danio rerio). Canadian Journal of Zoology, 81, 122-125. https://doi: 10.1139/Z02-229

Ribas, L., \& Piferrer, F. (2014). The zebrafish (Danio rerio) as a model organism, with emphasis on applications for finfish aquaculture research. Reviews in Aquaculture, 6, 209-240. https://doi.org/10.1111/raq.12041

Ribas, L., Liew, W. C., Díaz, N., Sreenivasan, R., Orbán, L., \& Piferrer, F. (2017). Heatinduced masculinization in domesticated zebrafish is family-specific and yields a set of different gonadal transcriptomes. Proceedings of the National Academy of Sciences of 
the United States of America, 114, E941-E950.

https://doi.org/10.1073/pnas.1609411114

SAS Institute. (2008). SAS/STAT®9.2User's Guide, the MIXED Procedure. SAS Institute Inc., Cary, NC.

SAS Institute. (2014). SAS Users's Guide: Statistics. Version 13.2. SAS Institute Inc., Cary, NC.

Schnurr, M. E., Yin, Y., \& Scott, G. R. (2014). Temperature during embryonic development has persistent effects on metabolic enzymes in the muscle of zebrafish. Journal of Experimental Biology, 217, 1370-80. https://doi:10.1242/jeb.094037

Scott, G. R., \& Johnston, I. A. (2012). Temperature during embryonic development has persistent effects on thermal acclimation capacity in zebrafish. Proceedings of the National Academy of Sciences of the United States of America, 109, 14247-52. https://doi:10.1073/pnas.1205012109

Serrana D. G. D. I, Vieira, V. L., Andree, K. B., Darias, M., Estévez, A., Gisbert, E., \& Johnston, I. A. (2012). Development temperature has persistent effects on muscle growth responses in gilthead sea bream. PLoS One, 7, e51884. https://doi: 10.1371/journal.pone.0051884

Singh, A. P., \& Nüsslein-Volhard, C. (2015). Zebrafish Stripes as a Model for Vertebrate Color Pattern Formation. Current Biology, 25, R81-R92. https://doi: 10.1016/j.cub.2014.11.013

Singh, R., \& Goel, M. K. (2016). Gender Classification Techniques-From Machine Learning to Deep Learning. International Journal of Computer Technology and Applications, 9, $77-88$.

Skinner, A. M. J., \& Watt, P. J. (2007). Strategic egg allocation in the zebra fish, Danio rerio. Behavioral Ecology, 18, 905-909. https://doi:10.1093/beheco/arm059

Spence, R., \& Smith, C. (2006). Mating preference of female zebrafish, Danio rerio, in relation to male dominance. Behavioral Ecology, 17, 779-783. https://doi:10.1093/beheco/ar1016

Stern, U., He, R., \& Yang, C. H. (2015). Analyzing animal behavior via classifying each video frame using convolutional neural networks. Scientific Reports, 5, 14351. https://doi: 10.1038/srep14351

Swain, M. J., \& Ballard, D. H. (1991). Color Indexing. International Journal of Computer Vision, 7, 11-32.

Toshev, A., \& Szegedy, C. (2013). DeepPose: Human pose estimation via deep neural networks. arXiv preprint arXiv:1312.4659 
Uusi-Heikkilä, S., Böckenhoff, L., Wolter, C., \& Arlinghaus, R. (2012). Differential allocation by female zebrafish (Danio rerio) to different-sized males--an example in a fish species lacking parental care. PLoS ONE, 7, e48317. https://doi: 10.1371/journal.pone.0048317

Valletta, J. J., Torney, C., Kings, M., Thornton, A., \& Madden, J. (2017). Applications of machine learning in animal behaviour studies. Animal Behaviour, 124, 203-220. https://doi.org/10.1016/j.anbehav.2016.12.005

Von Hertell, U., Hörstgen-Schwark, G., Langholz, H. J., \& Jung, B. (1990). Family studies on genetic variability in growth and reproductive performance between and within test fish populations of the zebrafish, Brachydanio rerio. Aquaculture, 85, 307-315. https://doi.org/10.1016/0044-8486(90)90029-M

Wacker, S., Östlund-Nilsson, S., Forsgren, E., Newport, C., \& Amundsen, T. (2016). Mate choice plasticity in a coral reef fish. Behavioral Ecology, 27, 1331-1342. https://doi.org/10.1093/beheco/arw050

Wang, G. (2019). Machine learning for inferring animal behavior from location and movement data. Ecological Informatics, 49, 69-76. https://doi.org/10.1016/j.ecoinf.2018.12.002

XU, Z., \& Cheng, X. E. (2017). Zebrafsh tracking using convolutional neural networks. Scientific Reports, 7, 42815. https://doi: 10.1038/srep42815 


\section{Appendix S1}

\section{Materials and methods}

\section{Color feature extraction}

For the color-based classification of sex, the images were first transformed from the RGB color space to the lab color space. Given the chromaticity coordinates of an RGB system $\left(\mathrm{x}_{\mathrm{r}}, \mathrm{y}_{\mathrm{r}}\right),\left(\mathrm{x}_{\mathrm{g}}, \mathrm{y}_{\mathrm{g}}\right)$ and $\left(\mathrm{x}_{\mathrm{b}}, \mathrm{y}_{\mathrm{b}}\right)$ together with its reference white $\left(\mathrm{X}_{\mathrm{W}}, \mathrm{Y}_{\mathrm{W}}, \mathrm{Z}_{\mathrm{W}}\right)$ such that

$$
\left[\begin{array}{l}
\mathrm{X} \\
\mathrm{Y} \\
\mathrm{Z}
\end{array}\right]=\mathrm{M}\left[\begin{array}{l}
\mathrm{R} \\
\mathrm{G} \\
\mathrm{B}
\end{array}\right]
$$

where

$$
M=\left[\begin{array}{lll}
S_{r} X_{r} & S_{g} X_{g} & S_{b} X_{b} \\
\mathrm{~S}_{r} Y_{r} & S_{g} Y_{g} & S_{b} Y_{b} \\
\mathrm{~S}_{r} Z_{r} & S_{g} Z_{g} & S_{b} Z_{b}
\end{array}\right],
$$

with $X_{r}=x_{r} / y_{r}, Y_{r}=1, Z_{r}=\left(1-x_{r}-y_{r}\right) / y_{r}, X_{g}=x_{g} / y_{g}, Y_{g}=1, Z_{g}$

$$
=\left(1-x_{g}-y_{g}\right) / y_{g}
$$

$X_{b}=x_{b} / y_{b}, Y_{b}=1, Z_{b}=\left(1-x_{b}-y_{b}\right) / y_{b}$ and

$$
\left[\begin{array}{c}
S_{r} \\
S_{g} \\
S_{b}
\end{array}\right]=\left[\begin{array}{ccc}
X_{r} & X_{g} & X_{b} \\
Y_{r} & Y_{g} & Y_{b} \\
Z_{r} & Z_{g} & Z_{b}
\end{array}\right]^{-1}\left[\begin{array}{c}
X_{W} \\
Y_{W} \\
Z_{W}
\end{array}\right]
$$

This representation then can be used to calculate the lab color space by:

$$
\begin{aligned}
\mathrm{L} & =116\left(\mathrm{f}_{\mathrm{y}}-16\right) \\
\mathrm{a} & =500\left(\mathrm{f}_{\mathrm{x}}-\mathrm{f}_{\mathrm{y}}\right) \\
\mathrm{b} & =200\left(\mathrm{f}_{\mathrm{y}}-\mathrm{f}_{\mathrm{z}}\right),
\end{aligned}
$$

where

$$
f_{i}=\left\{\begin{array}{c}
\sqrt[3]{i_{r}} \text { if } i_{r}>\epsilon \\
\frac{\kappa i_{r}+16}{116} \text { else }
\end{array} \text { Where } i_{r}=\frac{x}{x_{r}} \text { with } i \in\{x, y, z\}\right. \text { and }
$$

$\epsilon=0.008856, \kappa=903.3$ are defined by the CIE standards (McLaren, 1976). For every set of values of in $L, a, b$ a histogram is calculated, such that: $n=\sum_{i=1}^{k} m_{i}$ where $n$ is the number 
of observations and $k$ the total number of bins, for our study $k=255$. These histograms are used as features for a Support Vector Machine with a Gaussian kernel, we call the continuation of all three histograms $X_{i}$ with label $y_{i}$. In this way the training data is given as:

$$
\left\{\mathbf{X}_{\mathrm{i}}, \mathrm{y}_{\mathrm{i}}\right\}: \mathrm{i}=\{1, \ldots, 1\}, \mathbf{X}_{\mathrm{i}} \in \mathbb{R}^{\mathrm{n}}, \mathrm{y}_{\mathrm{i}} \in\{-1,1\}
$$

This data is supposed to be separated by a hyperplane: $\mathbf{X w}+\mathbf{b}=0$, where

- w normal to the hyperplane

- $\frac{|\mathrm{b}|}{|| \mathbf{w} \mid}$ is the distance to origin

- || $\mathbf{w}||$ Euclidean norm of $\mathbf{w}$

Such that:

$$
\begin{array}{ll}
\mathbf{X}_{\mathrm{i}} \mathbf{w}+\mathrm{b} \geq+1, & \mathrm{y}_{\mathrm{i}}=+1 \\
\mathbf{X}_{\mathrm{i}} \mathbf{w}+\mathrm{b} \leq-1, & \mathrm{y}_{\mathrm{i}}=-1 \\
\mathrm{y}_{\mathrm{i}}\left(\mathbf{X}_{\mathrm{i}} \mathbf{w}+\mathrm{b}\right)-1 \geq 0, \forall \mathrm{i}
\end{array}
$$

Since the distribution in the feature space of our data is not divisible by a linear hyperplane, we are assuming that:

$\boldsymbol{\Phi}: \mathbb{R}^{\mathrm{d}} \rightarrow H$,

Such that there is a kernel $\mathrm{K}$ :

$$
\mathrm{K}\left(\mathrm{x}_{\mathrm{i}}, \mathrm{x}_{\mathrm{j}}\right)=\boldsymbol{\Phi}\left(\mathrm{X}_{\mathrm{i}}\right) \boldsymbol{\Phi}\left(\mathrm{X}_{\mathrm{j}}\right) .
$$

By choosing a Gaussian kernel we get:

$$
\mathrm{K}\left(\mathbf{X}_{\mathrm{i}}, \mathbf{X}_{\mathrm{j}}\right)=\mathrm{e}^{\frac{-\left|\mathbf{X}_{\mathrm{i}}-\mathbf{X}_{\mathbf{j}}\right|^{2}}{2 \sigma^{2}}} .
$$

\section{Deep Convolutional Neural Networks}

The data processing through a network in convolutional neural networks is done layer wise. Considering $\mathbf{X}_{\alpha} \in \mathbb{R}^{\mathrm{h}_{\mathrm{X}}^{(0)} \times \mathrm{g}_{\mathrm{X}}^{(0)}}$ is an image with $\mathrm{h}_{\mathrm{X}}^{(0)}$ rows, $\mathrm{g}_{\mathrm{X}}^{(0)}$ columns, and $\mathrm{d}_{\mathrm{X}}$ channels (in our case of $L, a, b$ channels $d_{X}=3$ ). Each channel of $\mathbf{X}_{\alpha}$ is denoted by $X_{\alpha}^{(k)}$, such that $\mathbf{X}_{\alpha}=$ 
$\left\{\mathrm{X}_{\alpha}^{(1)}, \mathrm{X}_{\alpha}^{(2)}, \ldots, \mathrm{X}_{\alpha}^{(\mathrm{dx})}\right\}$. For each kernel $\mathrm{K}^{\mathrm{l}, \mathrm{d}} \in \mathbb{R}^{\mathrm{h}_{\mathrm{K}}^{1} \times \mathrm{g}_{\mathrm{K}}^{\mathrm{l}}}$ with size $\mathrm{h}_{\mathrm{K}}^{1} \times \mathrm{g}_{\mathrm{K}}^{1}$ and $\mathrm{d} \in\left\{1, \ldots, \mathrm{d}_{\mathrm{K}}\right\}$, the output $Y^{1} \in \mathbb{R}^{\frac{h_{X}^{1}-h_{K}^{1}-1+P^{1}}{s^{1}} \times \frac{g_{X}^{l}-g_{K}^{l}-1+P^{1}}{s^{1}}}$ is computed elementwise by:

$$
\mathrm{Y}_{\mathrm{x}, \mathrm{y}}^{\mathrm{l}, \mathrm{d}}=\left(\mathbf{X}^{1} * \mathrm{~K}^{\mathrm{l}, \mathrm{d}}\right)_{\mathrm{x}, \mathrm{y}}=\left(\mathrm{b}^{\mathrm{l}, \mathrm{d}}+\sum_{\mathrm{k}=1}^{\mathrm{d}_{\mathrm{x}}^{\mathrm{l}}} \sum_{\mathrm{i}=1}^{\mathrm{h}_{\mathrm{K}}^{\mathrm{l}}} \sum_{\mathrm{j}=1}^{\mathrm{gk}_{\mathrm{K}}^{\mathrm{k}}} \mathrm{K}_{\mathrm{i}, \mathrm{j}}^{\mathrm{l}, \mathrm{d}} \cdot \mathrm{X}_{\mathrm{x}+\mathrm{i}-1, \mathrm{y}+\mathrm{j}-1}^{\mathrm{l}, \mathrm{k}}\right) .
$$




\title{
CHAPTER 4
}

\section{Genetic mechanism underlying sexual plasticity and its association with colour patterning in zebrafish (Danio rerio)}

\author{
Shahrbanou Hosseini ${ }^{1,2}$, Ngoc-Thuy $\mathrm{Ha}^{1,2}$, Henner Simianer ${ }^{1,2}$, Clemens Falker- \\ Gieske $^{1,2}$, Bertram Brenig ${ }^{1,2,3}$, Andre Franke $^{4}$, Gabriele Hörstgen-Schwark ${ }^{1}$, Jens \\ Tetens $^{1,2}$, Sebastian Herzog ${ }^{5,6}$, Ahmad Reza Sharifi ${ }^{1,2}$ \\ ${ }^{1}$ University of Goettingen, Department of Animal Sciences, Goettingen, Germany \\ ${ }^{2}$ University of Goettingen, Center for Integrated Breeding Research, Goettingen, Germany \\ ${ }^{3}$ University of Goettingen, Institute of Veterinary Medicine, Goettingen, Germany \\ ${ }^{4}$ Institute of Clinical Molecular Biology, Christian-Albrechts-University, Kiel, Germany \\ ${ }^{5}$ Max Planck Institute for Dynamics and Self-Organization, Goettingen, Germany \\ ${ }^{6}$ University of Goettingen, Department for Computational Neuroscience, 3rd Physics Institute- \\ Biophysics, Goettingen, Germany
}

Published in BMC Genomics, 20:341

https://doi.org/10.1186/s12864-019-5722-1 


\begin{abstract}
Background: Elevated water temperature, as is expected through climate change, leads to masculinization in fish species with sexual plasticity, resulting in changes in population dynamics. These changes are one important ecological consequence, contributing to the risk of extinction in small and inbred fish populations under natural conditions, due to malebiased sex ratio. Here we investigated the effect of elevated water temperature during embryogenesis on sex ratio and sex-biased gene expression profiles between two different tissues, namely gonad and caudal fin of adult zebrafish males and females, to gain new insights into the molecular mechanisms underlying sex determination (SD) and colour patterning related to sexual attractiveness.
\end{abstract}

Results: Our study demonstrated sex ratio imbalances with $25.5 \%$ more males under hightemperature condition, resulting from gonadal masculinization. The result of transcriptome analysis showed a significantly upregulated expression of male SD genes (e.g. dmrt1, amh, cyp11c1 and sept8b) and downregulation of female SD genes (e.g. zp2.1, vtg1, cyp19ala and bmp15) in male gonads compared to female gonads. Contrary to expectations, we found highly differential expression of colour pattern (CP) genes in the gonads, suggesting the 'neofunctionalisation' of those genes in the zebrafish reproduction system. However, in the caudal fin, no differential expression of $\mathrm{CP}$ genes was identified, suggesting the observed differences in colouration between males and females in adult fish may be due to posttranscriptional regulation of key enzymes involved in pigment synthesis and distribution.

Conclusions: Our study demonstrates male-biased sex ratio under high temperature condition and support a polygenic SD (PSD) system in laboratory zebrafish. We identify a subset of pathways (tight junction, gap junction and apoptosis), enriched for SD and CP genes, which appear to be co-regulated in the same pathway, providing evidence for involvement of those genes in the regulation of phenotypic sexual dimorphism in zebrafish.

Keywords: zebrafish, temperature, embryogenesis, sex ratio, sex determination, colour pattern, masculinization, transcriptome analysis

\title{
Background
}

Mammals and avian species have a chromosomal sex determination (SD) mechanism in vertebrates with master switch of SD located on the sex chromosomes (Ribas et al., 2017; 
Liew and Orban, 2014; Liew et al., 2012; Cutting et al., 2013), whereas sex in teleost fish can be diverse and their sexual plasticity depends on genetic and environmental factors (Kobayashi et al., 2013). The genetic mechanism of SD in zebrafish (Danio rerio), a widely used model organism, is not fully understood, since there are no differences between the chromosomal sets of male and female genomes (Liew and Orban, 2014; Liew et al., 2012). In a previous study, it was found that a sex-associated region in zebrafish differs between wild and domesticated strains, located in the right telomere of chromosome 4 of wild populations. However, this sex-specific region was not found in domesticated strains (Wilson et al., 2014), leading to the assumption that sex in domesticated strain is polygenetically determined (Ribas et al., 2017; Liew et al., 2012; Wilson et al., 2014; Webster et al., 2017), in which the sexdetermining genes are distributed over the whole genome (Liew and Orban, 2014). Among a series of biotic and abiotic factors that influence the mechanism of SD in zebrafish during gonad development resulting in masculinization, temperature is the most important environmental factor (Ribas et al., 2017; Baroiller et al., 2009; Ospina-Alvarez et al., 2008). During early embryonic development, the number of primordial germ cells (PGCs) plays an important role in gonad differentiation and sexual dimorphism (Liu et al., 2015; Tzung et al., 2015). In order to form the primordial gonads, which later develop into a testis or an ovary, the PGCs form cell clusters and migrate during the first day of embryonic development toward the somatic cells of the gonad and merge with these cells to form germ cells. A subset of germ cells acquires the ability to operate as germ line stem cells, which later differentiate into gametes (Liu et al., 2015; Richardson and Lehmann, 2010). During this critical embryonic developmental period, the loss or decrease in the number of PGCs may cause by increased water temperatures leads to masculinization (Tzung et al., 2015; Abozaid et al., 2011; Hosseini et al., 2019). This process is regulated in such a way that the testicular developmental genes are expressed and the expression of the ovarian developmental genes is inhibited in the "juvenile ovary" stage (Baroiller et al., 2009; Siegfried and Nüsslein-Volhard, 2008). The sex-reversed females are known as "neomales", which possess testis and have similar gene expression profiles as normal males (Ribas et al., 2017). Hence, SD in zebrafish is controlled by the interaction between fish genotype and environmental factors $(\mathrm{GxE})$ (Ribas et al., 2017; Liew and Orban, 2014; Abozaid et al., 2011). Elevated water temperature, e.g. caused by climate change, may induce male-biased populations, leading to an elevated risk of extinction in thermosensitive fish populations in nature (Brown et al., 2015). 
In spite of the absence of heterogamety in zebrafish, sex-biased gene expression profiles in adult fish revealed a greater number of male-biased than female-biased genes and a higher magnitude of expression level in male-biased genes compared to female-biased genes in the gonadal tissues (Small et al., 2009; Yang et al., 2016). Furthermore, a greater proportion of sex-biased genes compared to unbiased genes in zebrafish demonstrated an evidence for positive selection and therefore faster evolution of sex-biased genes (Yang et al., 2016). In general, rapid evolution for sex-biased genes might be related to positive selection (Yang et al., 2016), sexual selection (Ellegren and Parsch, 2007) or to genetic drift (Dapper and Wade, 2016). Zebrafish does not have much more morphological sexual dimorphism. This can be explained by the fact that accelerated evolution for sex-biased genes as a consequence of sexual selection acts simultaneously on both male- and female biased genes (Yang et al., 2016). Nevertheless, male zebrafish show a more intense yellow colouration compared to females based on xanthophores, which is thought to be important for sexual attraction (Singh and Nüsslein-Volhard, 2015; Nüsslein-Volhard and Singh, 2017). Unlike mammals and birds, which have only one type of pigment cell (melanocytes), fish species have several types of chromatophores involved in the development of colour pattern (CP) (Singh et al., 2015; Mahalwar et al., 2016). The interaction within and between chromatophore cell types distributed in the hypodermis of the body and the epidermis of scales and fins are required for CP formation in zebrafish (Nüsslein-Volhard and Singh, 2017; Singh et al., 2015; Mahalwar et al., 2016). In the larval stage, chromatophores arise directly from neural crest cells, while adult stripe patterns are developed during metamorphosis (3-6 weeks post fertilization) and display golden and blue stripes composed of yellow xanthophores, silvery or blue iridophores and black melanophores (Singh and Nüsslein-Volhard, 2015; Nüsslein-Volhard and Singh, 2017; Volkening and Sandstede, 2015; Mahalwar et al., 2014; Walderich et al., 2016). Although the anal and caudal fin stripe patterns are contiguous with the body stripes, the mechanism of fin stripe formation differs from the body stripe formation. The mechanism involved in fin CP formation in zebrafish is still largely unexplored (Singh and NüssleinVolhard, 2015; Nüsslein-Volhard and Singh, 2017).

Despite the popularity of zebrafish as a well-established teleost research model animal, little information exists about the influence of elevated water temperature during embryonic development and its later effects on sex differentiation (Abozaid et al., 2011). In this study, we investigate the sex ratio in response to high water temperature during embryogenesis in respect to gonadal masculinization in zebrafish. Since the regulation of sex-biased gene 
expression plays a major role in phenotypic dimorphism (Small et al., 2009) and the expression of SD genes might be associated with $\mathrm{CP}$ genes in respect to sexual attraction (Sharma et al., 2014), the investigation of underlying molecular mechanisms of SD and CP genes and their interactions can provide new insights into the genetic control of sexual dimorphism in zebrafish. Furthermore, exposure to high ambient temperatures leads to a loss of pigmentation in domesticated zebrafish (Ribas et al., 2017). Transcriptome analysis of gonads and caudal fins was performed in this study to generate profiles of the global gene expression patterns in both sexes, due to the distinct phenotypic differences in colouration between males and females in the caudal fin of zebrafish (Hosseini et al., 2018). This will help fill the gap in the current knowledge regarding the association between SD and CP genes and the temperature effects on their expression in adult fish.

\section{Results}

\section{Temperature effects on sex ratio}

Exposure of zebrafish fertilized eggs during embryonic development from 5-24 hours post fertilization (hpf) to elevated water temperature resulted in a significantly higher male frequency compared to the control group ( $73.9 \%$ vs. $48.4 \%$; Figure 1$)$. The ratio of females in the treated group amounted to $26.1 \%$ as compared to the control group with $51.6 \%$. The $25.5 \%$ increase in the proportion of males under heated conditions compared to the control group suggests the induction of masculinization through the interaction between genotype and environmental factor (GxE) during SD and gonad differentiation. However, fish that do not change their sex under the influence of high temperatures are characterized as heatresistant female animals. 


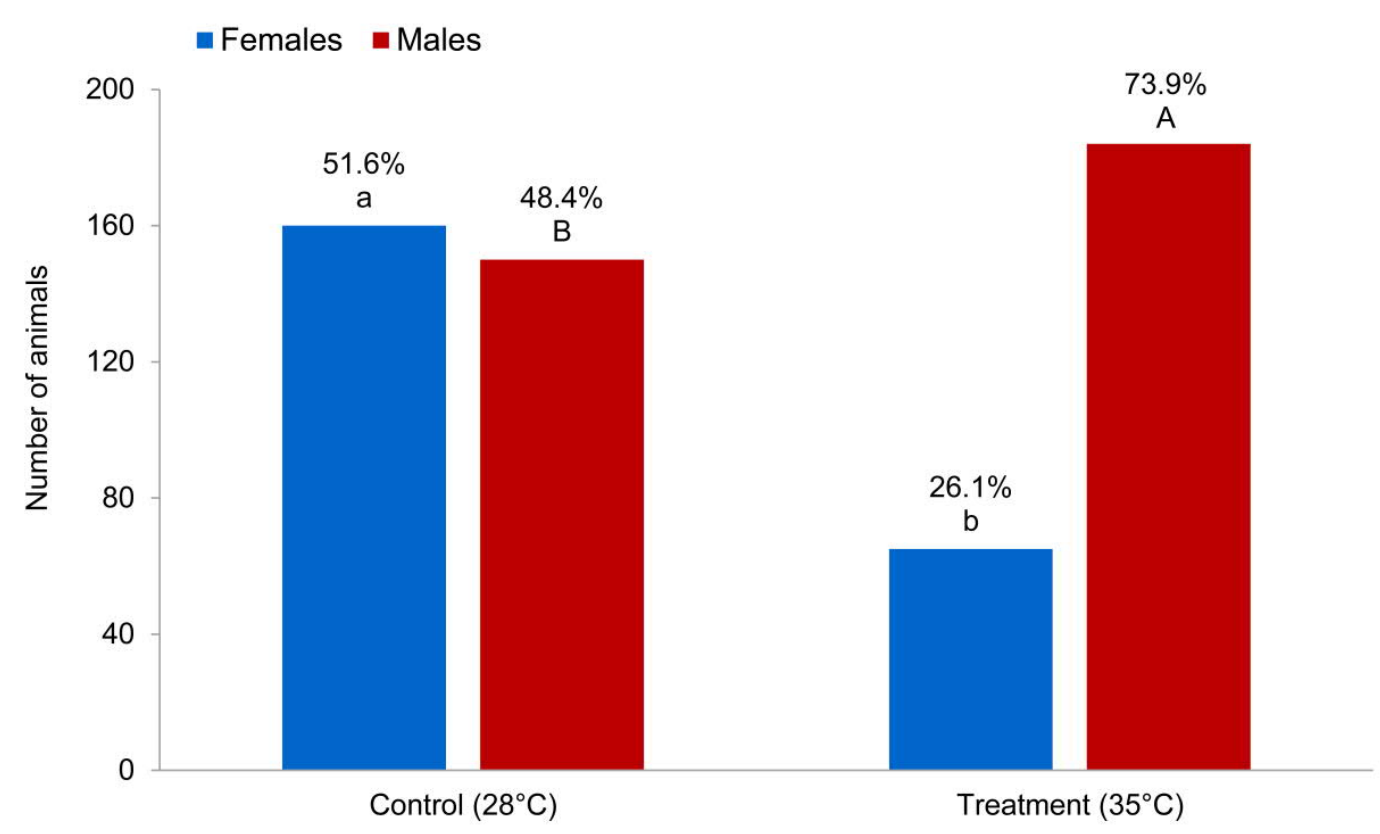

Figure 1: The effect of temperature treatment on sex ratio (Back-transformed least squares means in $\%$ using generalized linear model) is shown in control and temperature treatment groups. Means within treatment with different superscripts differ significantly $(\mathrm{P}<0.0001)$. ${ }^{\mathrm{A}-\mathrm{B}}$ Significant difference between proportion of males in control and temperature treatment, ${ }^{\mathrm{a}-\mathrm{b}}$ significant difference between proportion of females in control and temperature treatment

\section{Transcriptome analysis of differential gene expression in the gonads and caudal fins}

To elucidate the genetic mechanisms of association between SD and CP genes, we analysed the expression profiles of the gonad and caudal fin in treatment versus control group within males and females to find the effect of temperature treatment in different experimental groups (male treatment gonad vs. male control gonad: MTG vs. MCG; female treatment gonad vs. female control gonad: FTG vs. FCG; male treatment fin vs. male control fin: MTF vs. MCF and female treatment fin vs. female control fin: FTF vs. FCF), and males versus females within treatment and control groups to find the effect of sex in different experimental groups (male control gonad vs. female control gonad: MCG vs. FCG; male treatment gonad vs. female treatment gonad: MTG vs. FTG; male control fin vs. female control fin: MCF vs. FCF and male treatment fin vs. female treatment fin: MTF vs. FTF). A total number of 35,119 transcripts were read in RNA sequencing (RNA-Seq) expression profiles, in which the numbers of 18,871 expressed transcripts were analysed in all experimental groups. An 
overview of the significantly differentially expressed transcripts in comparison groups is presented in Table 1.

Table 1: Overview of the significantly differentially expressed transcripts in all compared experimental groups.

\begin{tabular}{cccc|cccc}
\hline & \multicolumn{3}{c}{ Treatment vs. Control } & \multicolumn{3}{c}{ Males vs. Females } \\
\hline \multicolumn{1}{c}{ Group } & Upregulated Downregulated & Total & Group & \multicolumn{2}{c}{ Upregulated Downregulated } & Total \\
\hline MTG vs. MCG & - & 31 & 31 & MCG vs. FCG & 7705 & 6355 & 14060 \\
FTG vs. FCG & - & - & - & MTG vs. FTG & 6483 & 6449 & 12932 \\
MTF vs. MCF & - & - & - & MCF vs. FCF & 247 & 172 & 419 \\
FTF vs. FCF & 16 & 10 & 26 & MTF vs. FTF & 334 & 467 & 801 \\
\hline
\end{tabular}

\section{Differentially expressed genes in temperature treatment versus control}

The results of the treatment versus control comparison in male and female gonads (Figure 2, Additional file 1) showed no significantly differentially expressed genes (DEGs) in FTG compared to FCG. However, 31 genes were down-regulated in MTG vs. MCG, where most of them play important role in kidney, liver, pancreas and gonad development (e.g. ela2, ela2l, ela3l and wt1b). This results revealed that one paralog of wilms tumor suppressor 1 (wt1b) (SD gene, Additional file 2; see explanation in the methods) is significantly downregulated in MTG compared to MCG, whereas this gene is up-regulated in the FTF compared to the FCF. In general, Wtl encodes a zinc finger transcription factor, which is necessary for the development of different tissues including kidney, gonad, spleen and heart in fish (Schnerwitzki et al., 2014). Both paralogous of wtl (wtla and wtlb) are existed in zebrafish (Bolling et al., 2006; Perner et al., 2007).

In contrast to the gonad, the transcriptome analysis in the caudal fins demonstrated no DEGs in MTF vs. MCF, while 26 significant DEGs (16 up-regulated and 10 down-regulated) were observed in FTF compared to FCF. The most significant down-regulated genes in FTF are osteocalcin genes, which are involved in mineralization of caudal fin rays and fin skeleton formation (e.g. bglapl, f13a1 and plod1a) in zebrafish (Bensimon-Brito et al., 2012; Carvalho et al., 2017). 

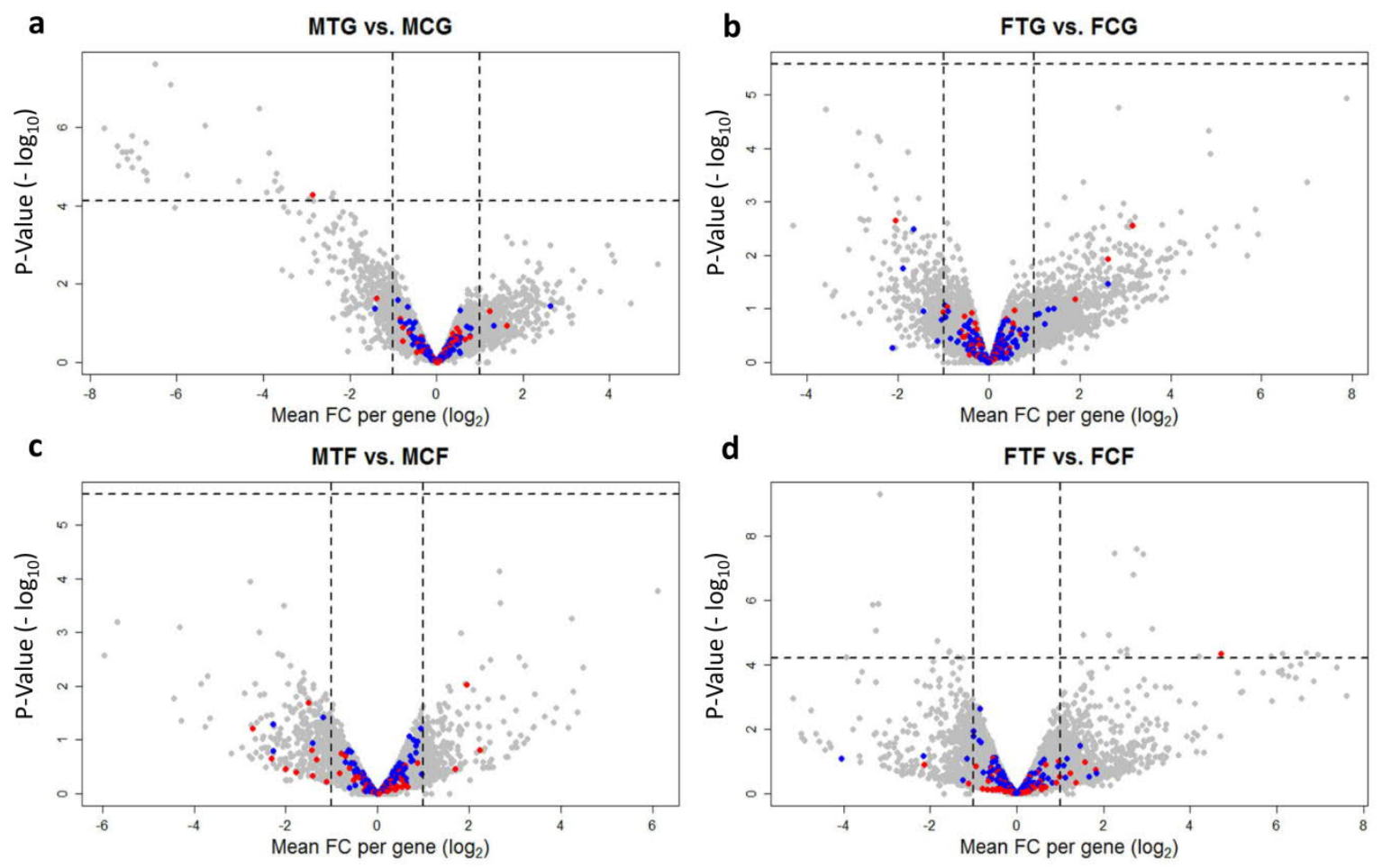

Figure 2: Volcano plots illustrate significantly differentially expressed genes (DEGs) in treatment versus control within males and females in the gonad and caudal fin. a, male treatment gonad versus male control gonad: MTG vs. MCG; $b$, female treatment gonad versus female control gonad: FTG vs. FCG; c, male treatment fin versus male control fin: MTF vs. MCF and d, female treatment fin versus female control fin: FTF vs. FCF. Each dot in the plot represents a gene with its corresponding $\log 2-$ fold change (FC) on the $\mathrm{x}$-axis and p-value $(-\log 10)$ on the $\mathrm{y}$-axis. Red colour dots show selected candidate sex determination genes and blue colour dots represent selected candidate colour pattern genes. The horizontal line indicates the significance threshold (false discovery rate; FDR $<0.05$ ), while the vertical line segregates genes with $\log 2 \mathrm{FC}>1$.

The upregulation of the myosin heavy chain isoforms gene (myhc4), which plays a role in muscle cells (Bryson-Richardson et al., 2005), is identified in FTF vs. FCF. We also found upregulation of a small heat shock protein (hspbl1) in FTF compared to FCF, indicating the physiological response of animals to the high ambient temperature in treatment compared to the control group. This gene is expressed during development and its expression level promotes resistance to environmental stressors. Thirteen small heat shock proteins (sHSPs) are identified in zebrafish, in which most of them are reported to be up-regulated during development under environmental heat shock (Elicker et al., 2007). 


\section{Differentially expressed genes in male versus female gonads}

The result of male versus female comparison in treated and non-treated groups in the gonad and caudal fin resulted in a considerable number of DEGs, illustrating the effect of sex on transcriptome profiles within treatment groups (Figure 3, Additional file 1). The differential expression level of candidate SD and CP genes (Additional file 2; see explanation in the methods) in the gonad and caudal fin are illustrated in Figure 3. A set of significantly DEGs from both gene groups in the gonads is presented in Figure 4. As expected, a substantial number of up-regulated male-biased SD genes (e.g. dmrt1, amh, gsdf, tuba7l and sox9a) were identified in MCG vs. FCG and MTG vs. FTG (Figure 4). The result showed that the malebiased genes related to steroidogenesis (cyp11c1, esr2b, hsd11b2, star and cyp1la2) and spermatogenesis (klhllOa, odf3b and tekt1) are highly up-regulated in our transcriptome profiles in treated and non-treated groups. The p53 signalling pathway-involved genes (tp53 and $d k k 3 b)$ responsible for testicular differentiation and a TNF-related apoptosis gene (tnfsflol) are up-regulated in male gonad in both temperature treated and control groups. A greater magnitude of septin signaling transcripts (sept3 and sept8b) encoding sperm tail proteins was identified in the testis. A similar high expression mode was observed for the spermatocyte development gene (cycp3). We also detected an upregulation of leydig cell (pdgfra) and sertoli cell differentiation $(\operatorname{sox} 9 a)$ genes in the testis. In contrast to the upregulation of male-biased genes, down-regulated expression patterns of female-biased SD genes (e.g. cyp19ala, figla, gdf9 and Bmp15) were observed in MCG vs. FCG and MTG vs. FTG (Figure 4). Our transcriptome analysis demonstrated that the most important folliculogenesis (bmp15, figla, gdf9 and lhx8a), vitellogenesis (vtgl and vtg5) and zona pellucida proteins genes for oogenesis (zp2.1 and $z p 3 b)$ are highly expressed in the ovary. In addition, steroid hormone and prostaglandin signalling genes (cypllal and cyp19ala) and Wnt signalling pathway genes (ctnnbip1, lef1 and axin1) in the ovary were also observed to be up-regulated as compared to the testis in this study. Contrary to expectations, we observed a set of significant differentially expressed CP genes in the gonad (MCG vs. FCG and MTG vs. FTG, Figure 4), even though these were not differentially expressed in the caudal fin, which could be due to their multifunctional character in different tissues. 
a

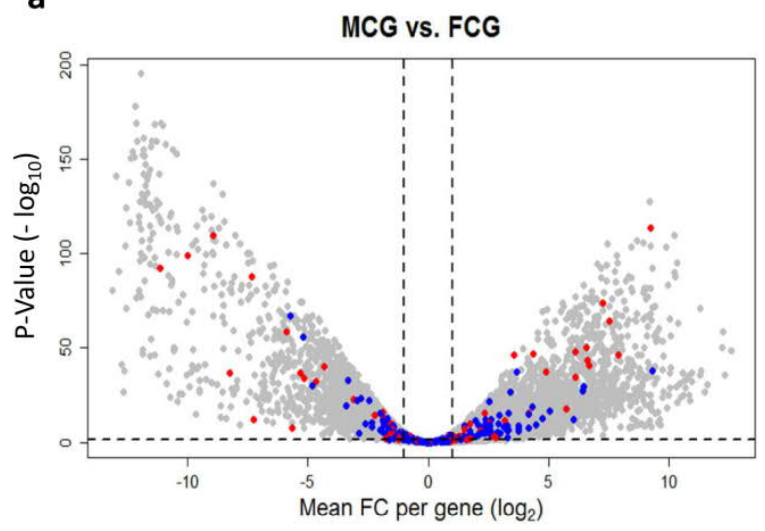

c

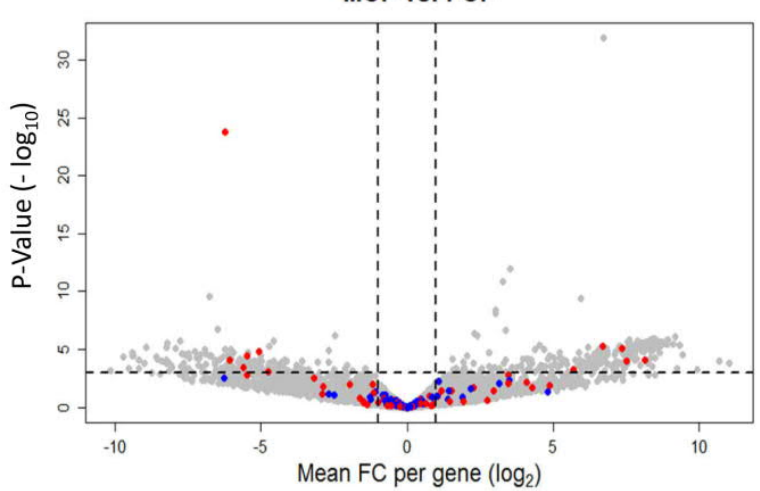

b

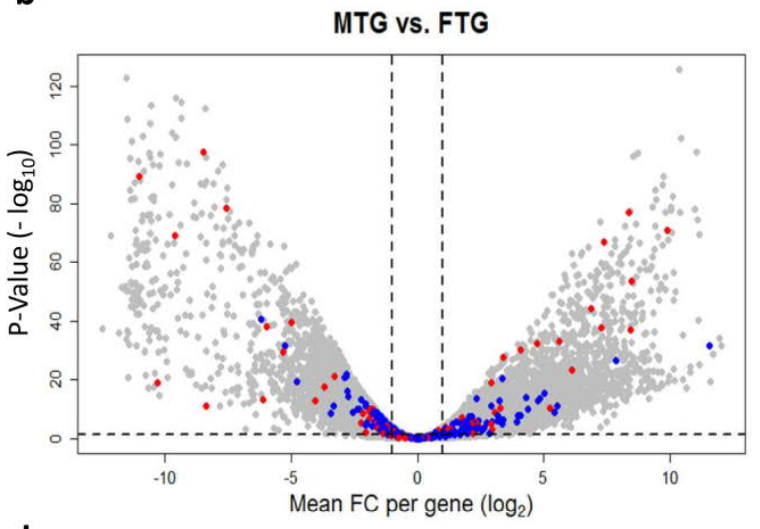

d

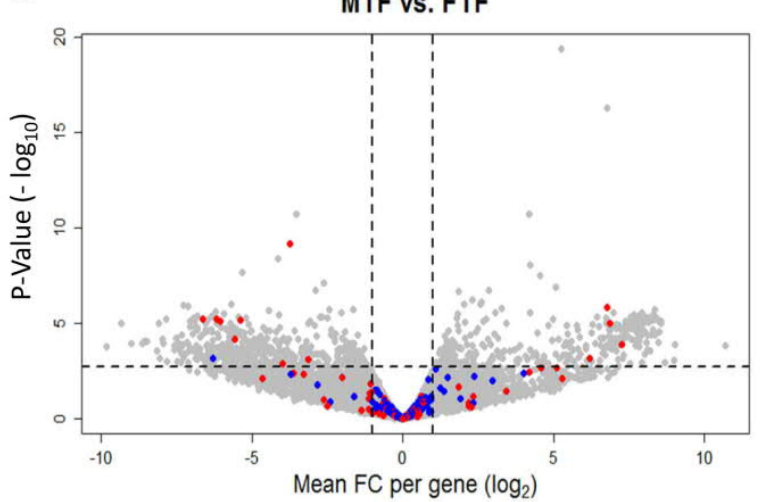

Figure 3: Volcano plots illustrate the result of significantly differentially expressed genes (DEGs) in males versus females within treatment and control groups in the gonad and caudal fin. a, male control gonad versus female control gonad: MCG vs. FCG; b, male treatment gonad versus female treatment gonad: MTG vs. FTG; c, male control fin versus female control fin: MCF vs. FCF; d, male treatment fin versus female treatment fin: MTF vs. FTF. Each dot in the plot represents a gene with its corresponding $\log _{2}$-fold change (FC) on the $\mathrm{x}$-axis and p-value $\left(-\log _{10}\right)$ on the $\mathrm{y}$-axis. Red colour dots show selected candidate sex determination genes and blue colour dots represent selected candidate colour pattern genes. The horizontal line indicates the significance threshold (false discovery rate; $\mathrm{FDR}<0.05)$, whereas the vertical line segregates genes with $\log _{2} \mathrm{FC}>1$. 


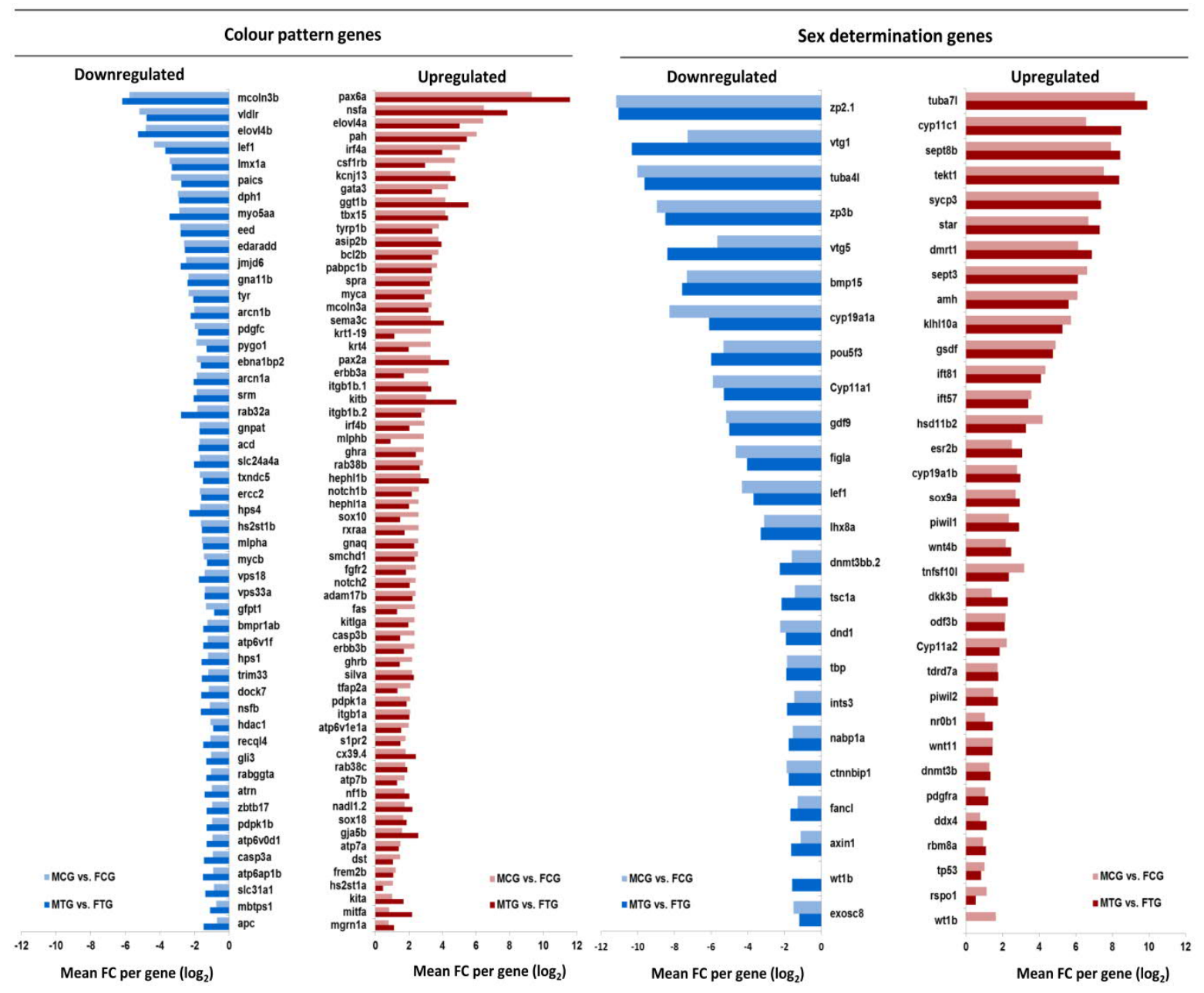

Figure 4: Bar charts illustrate up- and downregulation of selected candidate genes for sex determination and colour pattern in male versus female gonads within treated and non-treated groups: male control gonad (MCG), female control gonad (FCG), male treatment gonad (MTG), and female treatment gonad (FTG). The $x$-axis represents $\log _{2}$-fold change (FC>1) of expressed genes, which has been shown in the threshold value of false discovery rate $(\mathrm{FDR}<0.01)$.

\section{Differentially expressed genes in male versus female fins}

In contrast to the gonad, the $\mathrm{CP}$ genes in the caudal fin were not significantly differentially expressed in males versus females in treated and non-treated groups (Figure 3), while a high expression magnitude of some CP genes in different groups (MCF, MTF, FCF and FTF) was observed belonging to higher than the 75 quantile of CPM (counts per million reads) distribution ( $\log _{2} \mathrm{CPM}$ per mega base pairs; $\mathrm{Mb}, 24 \%$ of selected candidate $\mathrm{CP}$ genes in the transcriptome profile; Figure 5, Additional file 2). Certain candidate CP genes, such as rsp20, 
krt4, rps19, rpl24 and pabpcla were expressed at the highest level in all aforementioned groups (higher than 99 percentile; $\log _{2} \mathrm{CPM}$ per $\mathrm{Mb} \sim 18.5$ ).

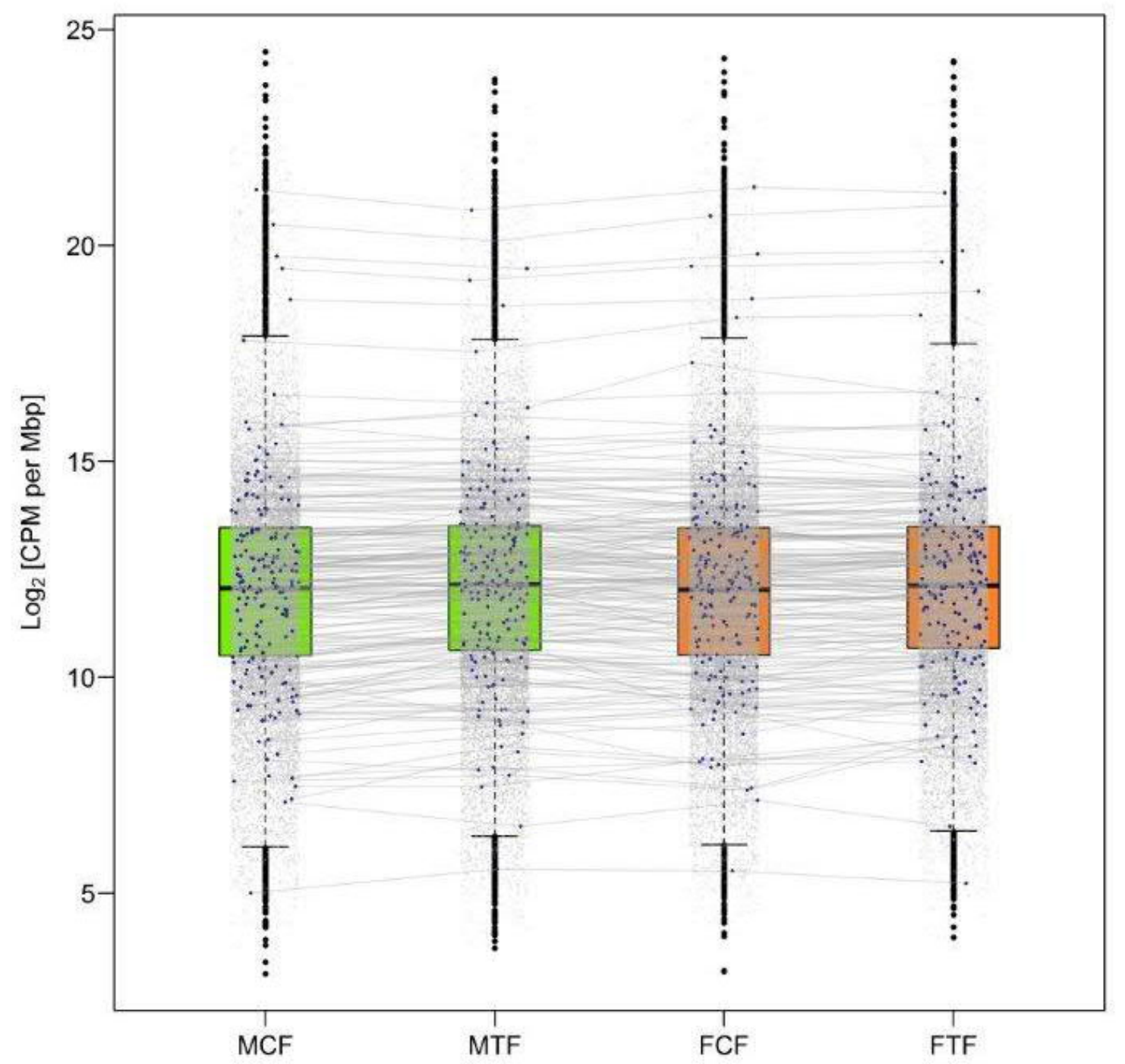

Figure 5: Box plots illustrate the expression of selected candidate colour pattern genes (CP) in the caudal fin. Each dot in the plot represents level of expressed gene in transcriptome profiles of four experimental groups. Blue dots show the expression level of selected candidate CP genes in male control fin $(\mathrm{MCF})$, male treatment fin $(\mathrm{MTF})$, female control fin $(\mathrm{FCF})$, and female treatment fin (FTF), which are not significantly differentially expressed. Each blue dot in different experimental groups stands approximately on the same expression level and the expression level of corresponding dot in different experimental group connected using gray lines. The y-axis is the logarithm of counts per 1 million reads per mega base pairs ( $\log _{2} \mathrm{CPM}$ per Mbp). 
The selected candidate CP genes in this study (Additional file 2) expressed in the caudal fin were classified based on their physiological function consisting of: (1) melanophore development, e.g. itgbla, hdacl, mitfa, kitlga and kita; (2) components of melanosomes, e.g. silva, tyr, slc24a4 and tyrp1b; (3) melanosome construction, e.g. vps18, nsfa, hps1 and vps33a; (4) melanosome transport, e.g. mlpha and myo5aa; (5) regulation of melanogenesis, e.g. asip2b and mgrnla; (6) systemic effects, e.g. elovl4b, vldlr, casp3a, atp7b and atp6ap1; (7) xanthophore development, e.g. csflra, ghrb and sox10; (8) Iridophore development, e.g. ltk, hdacl, tjpla, tjplb and vps18; (9) pteridine synthesis, e.g. paics, frem $2 b$ and spra, in carotenoid-based colour patches; and (10) Eumelanin and Pheomelanin, e.g. sox18, nadl1.2 and edaradd.

\section{Gene set enrichment and pathway analysis of differentially expressed genes}

A Venn diagram in Figure 6 displays the overlapping associations between significantly DEGs of comparison experimental groups (MCG vs. FCG, MTG vs. FTG, MCF vs. FCF and MTF vs. FTF). In total, 762 significantly DEGs were identified in MTG vs. FTG and 119 in MTF vs. FTF $\left(\right.$ FDR $<0.005, \log _{2}$ FC $\left.>2\right)$, which were not overlapping with their corresponding control groups, MCG vs. FCG and MCF vs. FCF, respectively. In the overlapping sets, 108 significantly DEGs (FDR $<0.005, \log _{2} \mathrm{FC}>2$ ) were detected between four comparison groups, considering the genes involved in the gonads and caudal fins. The lists of these genes were used for Gene Ontology (GO)-enrichment in the biological process category and pathway analysis (Additional file 3). The result demonstrated that the enriched GOs in the caudal fin have functional roles in the cilia and flagella structure in the surface of the cells such as cilium movement (GO:0003341), motile cilium assembly (GO:0044458) and ciliumdependent cell motility (GO:0060285). Their corresponding enriched pathways play a role in isoprenoid biosynthesis to chondrocytes and cartilage tissues development, such as terpenoid backbone biosynthesis, synthesis and degradation of ketone bodies, and valine leucine and isoleucine degradation (Figure 7a Set A, 7b Set A, Additional file 3). The Wnt signalling pathway involved in somitogenesis (GO:0090244), zona limitans intrathalamica formation (GO:0022006), microtubule-based process (GO:0007017) and eye pigment granule organization (GO:0008057), and the relevant pathways in ovarian and testis development namely Fanconi anemia and apoptosis were enriched in the gonad (Figure 7a Set B, 7b Set B, Additional file 3). 


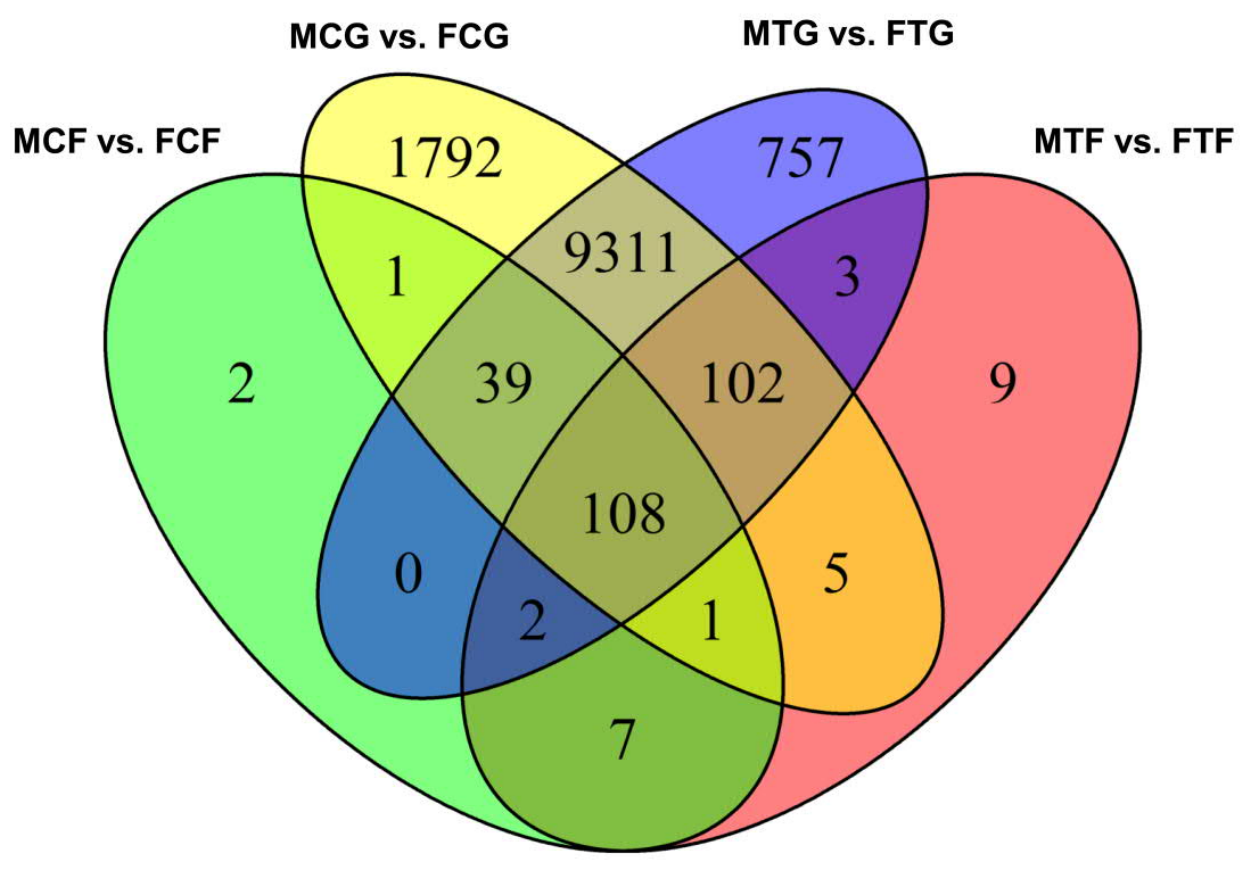

Figure 6: Venn diagram shows overlapping of significantly differentially expressed genes (DEGs) (false discovery rate; FDR $<0.005)$ using multiple comparisons with fold-changes $(\mathrm{FC}>2)$ : male control gonad versus female control gonad (MCG vs. FCG, yellow), male treatment gonad versus female treatment gonad (MTG vs. FTG, blue), male control fin versus female control fin (MCF vs. FCF, green), and male treatment fin versus female treatment fin (MTF vs. FTF, red).

More importantly, the result of four comparison groups illustrated the enriched GO terms related to SD and $\mathrm{CP}$, such as binding of sperm to zona pellucida (GO:0007339), positive regulation of acrosome reaction (GO:2000344), egg coat formation (GO:0035803) and oogenesis (GO:0048477) for SD and lateral semicircular canal development (GO:0060875) and retinal pigment epithelium development (GO:0003406) for $\mathrm{CP}$ formation, in respect to biological processes.

According to pathway analysis in this group, tight junction was the top significant pathway (FDR $\leq 0.002$ ) including important differentially expressed SD genes, tuba4 (Knoll-Gellida et al., 2006) and tuba7l (Santos et al., 2007), which are functionally involved in ovarian follicle and in testis development, respectively. The same SD genes (tuba4l and tuba7l) were also observed in the enriched gap junction and apoptosis pathways. 
a

$\left.\begin{array}{r}\text { GO Terms } \\ \text { microtubule-based movement } \\ \text { cilium movement } \\ \text { steroid biosynthetic process } \\ \text { axonemal dynein complex assembly }\end{array}\right]$ Set A

b

KEGG enriched pathways

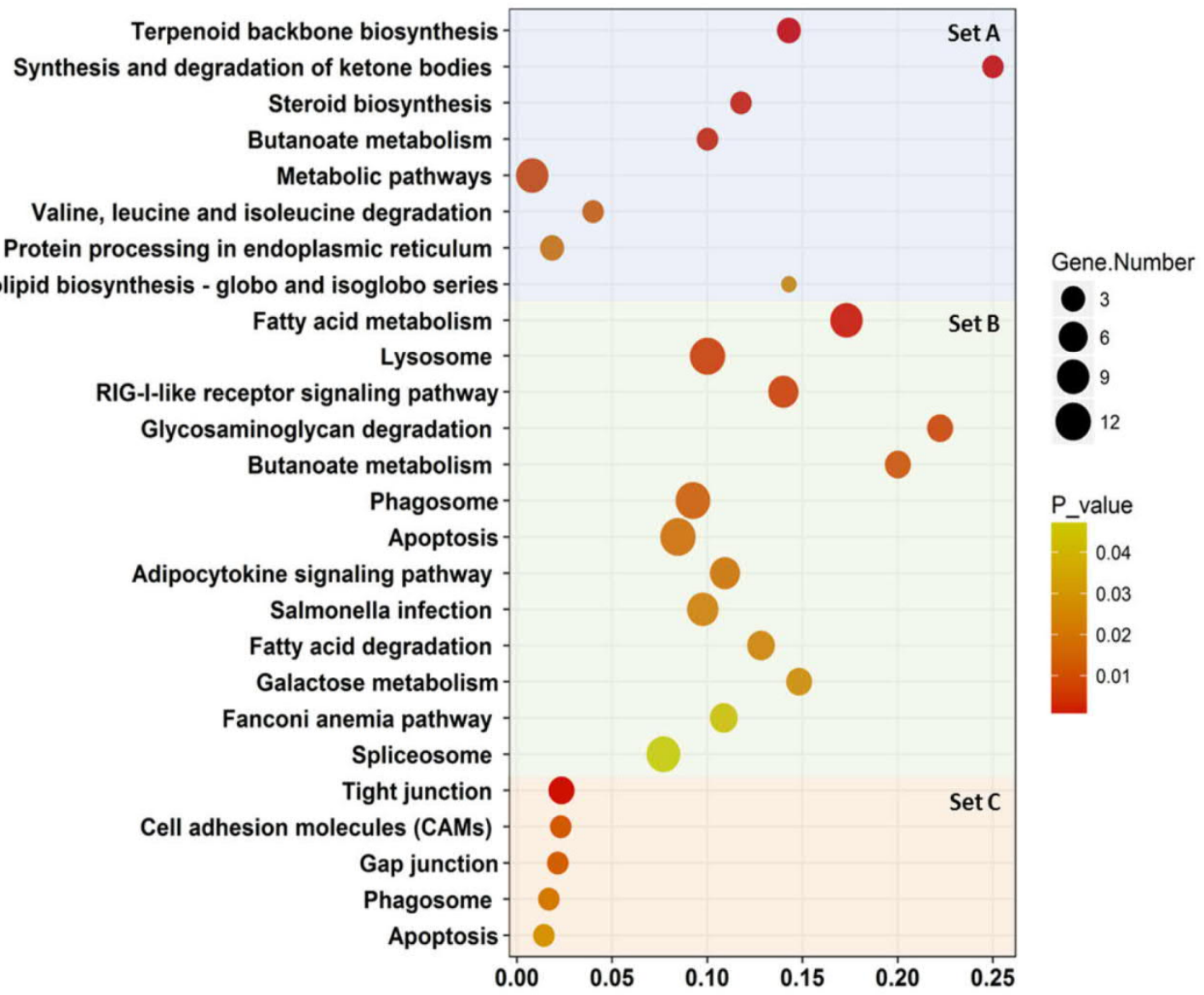


Figure 7: Results of gene set enrichment analysis (GSEA): a, Bar charts represent classification of significantly differentially expressed genes (DEGs) in gene ontology (GO) database. Categories are indicated using the number of genes present in each category and the number of genes identified in the GO library $(\mathrm{p}<0.05)$. The vertical axis represents 5 top ranks GOs with the smallest $\mathrm{p}$-values and in addition $3\left(^{*}\right)$ interesting GOs regarding our objectives in this study, and the horizontal axis represents the number of significantly DEGs in each GO term. b. Scatter plots illustrate enriched KEGG database pathways. The vertical axis represents the enriched pathway categories and the horizontal axis represents the rich factor of the enriched pathways. The size and colour of dots represent the gene number and the range of p-values, respectively. Rich factor is the ratio of differentially expressed gene number enriched in the pathway to the total gene number in a certain pathway. Set A: the enriched pathways or GOs considering significantly DEGs in MTF vs. FTF (119 genes), Set B: the enriched pathways or GOs considering significantly DEGs in MTG vs. FTG (762 genes), Set C: the enriched pathways or GOs considering significantly DEGs in overlapping between four comparison groups (108 genes).

The genes in the tight junction pathway with a CP function in melanophore development and migration (itgbla and itgblb.2) and iridophore migration or shape change (tjpla and tjplb) (Fadeev et al., 2015) are highly expressed in our transcriptome profiles of the caudal fin. Likewise, other $\mathrm{CP}$ genes ( $p d g f c$, gnalla, and map2kl) with a similar function in melanophore development in the gap junction pathway and the genes with systemic effects (casp3a, casp3b and $p d p k 1 b$ ) in the apoptosis pathway were also expressed in the caudal fin in this study (Figure 7a Set C, 7b Set C, Additional file 3). Therefore, the gene set enrichment analysis (GSEA) in this study exhibited the enriched pathway genes play a role in the SD and $\mathrm{CP}$ and may have a co-regulation mechanism resulting in co-expression of those genes as expected.

\section{Discussion}

\section{Sex ratio in response to high temperature}

In this study, high water temperature treatment during embryogenesis in zebrafish resulted in masculinization, while in the control group no significant difference was found between male and female proportion. In a comparable study, the effect of elevated water temperature (at $35^{\circ} \mathrm{C}$ during embryogenesis) from a mating of mitotic gynogenic males with normal females was investigated in zebrafish (Abozaid et al., 2011). A mitotic gynogenetic male is developed 
by applying a shock treatment during the first embryonic cell cycle of a maternal haploid cell that induces two sets of chromosome in the same nucleus and forms a diploid cell. In femaleheterogametic SD system, the gynogenesis process will result in an equal number of ZZ males and WW females, assuming that WW individuals are viable. However, if the W chromosome is lethal in the homozygous condition, all offspring will be male (Devlin and Nagahama, 2002). In F1-generation of crossing between the mitotic gynogenetic males and normal females the expected proportion of females would be $50 \%$ (in the case of using $\mathrm{ZZ}$ as a father) or $100 \%$ (in the case of using sex-reversed WW male as a father) (Abozaid et al., 2011). However, the result of the aforementioned study (Abozaid et al., 2011) revealed a high male frequency in temperature treatment (47.5\%) compared to the control group (22\%), which indicates the influence of the high ambient temperature on masculinization. A recent study of heat-induced masculinization in domesticated $A B$ strain of zebrafish showed that elevated water temperature $\left(36^{\circ} \mathrm{C}\right)$ during larval stage leads to a wide variety of inter-family masculinization up to $90 \%$ (Ribas et al., 2017). The results of the effect of the increase in water temperature on sex ratio in previous studies (Ribas et al., 2017, Abozaid et al., 2011, Hosseini et al, 2019) are in agreement with the outcome of this study. In many other fish species, which have a similar sexual plasticity, the effect of elevated water temperature on masculinization has been demonstrated (Díaz and Piferrer, 2015, Selim et al., 2009, Rougeot et al., 2008). Therefore, our result emphasizes the interplay between genetic and environmental influences on SD in zebrafish and confirms earlier studies on polygenic SD (PSD) in domesticated strains (Liew and Orban, 2014; Liew et al., 2012; Anderson et al., 2012) during embryonic (Abozaid et al., 2011, Hosseini et al, 2019) and larval stages (Ribas et al., 2017). However, little is known about the molecular basis of the effects of high water temperature during embryogenesis on SD in zebrafish and many closely related species.

\section{Transcriptome profiles of differentially expressed genes in different temperature groups}

Transcriptome analysis of gonads and caudal fins were performed to investigate the underlying genetic mechanism of the association between SD and CP genes and the effect of high ambient temperature on their expression profiles in zebrafish. Gene expression analysis in treatment versus control group within males and females is showed a set of DEGs in MTG vs. MCG and in MTF vs. MCF. One of these genes, Wt1, is down-regulated in MTG vs. 
MCG, which is related to the development of different organs in zebrafish. Most studies on the function of wt 1 in zebrafish have been focused on the embryonic kidney, the pronephros, development (Bolling et al., 2006; Perner et al., 2007). However, Wt1 is necessary for urogenital ridge development and identifying the adrenal-gonadal primordium (AGP), and both paralogous are seems to be required for kidney and gonad development in zebrafish (Chou et al., 2017). These paralogous have a clear role in pronephric glomerular formation of kidney during embryonic development in zebrafish. Wtla is expressed at the early of pronephros development to form glomerular structures, while $w t 1 b$ is expressed in the later stage of nephrogenesis (Perner et al., 2007). In adult zebrafish, a high expression level of both wtl genes can be found in the kidney, gonad, heart, spleen, and muscle tissues (Schnerwitzki et al., 2014; Bolling et al., 2006). Expression of wt1 at AGP is essential for the steroidogenic interrenal cells development in cooperation with fflb (nr5ala) gene (the equivalent of mammalian $S F-1$ ), suggesting its important role during development of gonadal primordium in zebrafish (Chou et al., 2017, Hsu et al., 2003, von Hofsten and Olsson, 2005). In a recent study, expression of wtla gene was observed in the zebrafish testis and is identified as a pro-male gene (Lee et al., 2017). In medaka, wtla is expressed in the somatic cells of the gonadal primordium, but wt $1 b$ is expressed in the later stage of development. The somatic gonadal cells in medaka arise from the lateral plate mesoderm (LPM) at the early of embryonic development (Nakamura et al., 2006). Wt1a is expressed in the LPM during early embryogenesis and later in the somatic cells of the primordial gonad. Wt1b coordinates with the wtla to develop both pronephros and gonad developments. Expression of both genes in the medaka displays a strong effect on the maintenance and survival of the number of PGCs during gonad development (Klüver et al., 2009). Our transcriptome profiles revealed that thermal treatment during embryogenesis might influence the expression mechanism of the $w t 1 b$ gene in the zebrafish during gonad differentiation, which in turn may result in masculinization. Since the number and survival of PGCs are necessary for ovarian development in zebrafish (Liu et al., 2015; Tzung et al., 2015), we hypothesized that downregulation of wt $1 b$ in MTG compared to MCG during embryogenesis in our study may cause the reduction in the number of PGCs, leading to masculinization in heat-treated animals. However, its underlying molecular mechanism requires further research. Furthermore, the high expression of hspb11 in FTF vs. FCF with regard to the thermal stress has been observed in this study. Generally, sHSPs act as molecular chaperones to prevent the aggregation of denatured proteins or to reverse improper protein associations during cellular 
stress (Marvin et al., 2008; Stengel et al., 2010; Klüver et al., 2011). This function is an important physiological role of sHSPs, which implicitly induces of the expression of sHSPs by a variety of stressors (Stengel et al., 2010; Klüver et al., 2011). In zebrafish, the high expression of $h s p b 11$, as a member of sHSPs family, was observed in somites, heart, dorsal mid- and hindbrains after heat shock (Elicker et al., 2007; Marvin et al., 2008). In this study, we observed a high expression of hspbl1 in the caudal fin of zebrafish in response to the elevated water temperature.

\section{Transcriptome profiles of differentially expressed genes in different sexes}

Within a substantial number of significantly differently expressed sex-biased genes, a series of pro-male and pro-female genes were identified within two temperature groups. One of the most important pro-male genes is $d m r t 1$, which has a sex-specific role in testis development and its expression is necessary for the transcriptional regulation of $a m h$. Amh is a key testis gene and normally expressed in the sertoli cells after testicular differentiation and inhibits the ovarian aromatase gene expression (cyp19ala), resulting in gonadal masculinization in zebrafish. Amh is downstream of $d m r t 1$ in zebrafish and its expression is regulated by $d m r t 1$ in somatic cells of testis. These suggest that $d m r t 1$ plays a male-specific role in zebrafish and loss or decrease of its expression interferes with normal male sexual development (Webster et al., 2017; Lee et al., 2017, Wang and Orban, 2007). A similar expression pattern of $d m r t 1$ in other fish species such as medaka (Hattori et al., 2007), tilapia (Poonlaphdecha et al., 2013), European seabass (Díaz and Piferrer, 2015) and pejerrey fish (Fernandino et al., 2008) has been observed in temperature-induced masculinization. Furthermore, steroidogenic enzymes, encoded by cypl1cl and hsd11b2 pro-male genes (Lee et al., 2017), are required for 11oxygenated androgen production, which are up-regulated in testis in this study. In contrast to the pro-male genes, cyp19ala is a key regulator gene in ovarian development (pro-female gene) encodes the P450 aromatase enzyme, responsible for conversion of androgens to estrogens in the female gonad, and is a downstream gene of Bmp15. Oocyte-produced signalling protein, Bmp15, is necessary for expression of cyp19ala and maintains of adult female sex differentiation in zebrafish. Hence, loss or downregulation of the expression of Bmp15 leads to a reduction in the expression of cyp19ala and consequently to a disruption of ovarian development (Dranow et al., 2016). Additionally, the expression of zp family member genes ( $z p 2$ and $z p 3$ ), which are up-regulated in the ovary, encodes the major protein components of zebrafish egg chorion (glycoprotein layer) and is active in the development of 
oocytes (Mold et al., 2009). Finally, a high number of significantly sexually dimorphic transcripts are identified in our study (Table 1), in which some of them were already identified in other studies as pro-male and pro-female genes (Ribas et al., 2017; Lee et al., 2017) in accordance with our results. During the zebrafish SD and gonad differentiation, the actions of several pathways regulate the sexual fate of an organism to develop either a testis or an ovary (Santos et al., 2017). GSEA in this study revealed that Fanconi anemia and apoptosis pathways with SD function in the ovary and testis respectively were enriched in the comparative analysis of adult gonads, supporting the observation of previous studies (Rodríguez-Marí et al., 2011; Ribas et al., 2017).

Surprisingly, similar to the sex-biased gene expression in the gonads, a significant set of $\mathrm{CP}$ genes was differentially expressed in the gonads. Many of the CP genes involved in pigment cell development have other functions not related to the pigmentation and they are considered as duplicated genes that have arisen from ancestral genome duplication specific to ray-finned fish (Braasch et al., 2009). A study on retroduplication in mammals and Drosophila demonstrated that "testis" has a central role in fixation and functional evolution of new genes. Indeed, testis is an evolutionary tissue with the most rapidly evolving organ, which may represent the target tissue for the evolution of new genes (Betrán et al., 2002; Kaessmann, 2010). These genes and their functions could become adopted into other tissues over time (Kaessmann, 2010; Heinen et al., 2009). These novel genes can impact the evolution of cellular, physiological, morphological, behavioral and reproductive phenotypic traits (Kaessmann, 2010). The neofunctionalisation of one copy of many duplicated genes implicated a secondary character, but is interestingly co-opted to a primary role after duplication (Conant and Wolfe, 2008). The kit system is a well-studied duplicated gene family known to play an essential role in pigmentation and ovarian development. Kitlga and kita genes are more specialized for melanophore development and migration (Hultman et al., 2007; Dooley et al., 2013). Kit ligand and their receptor also play an important role in spermatogenesis and oogenesis in adult zebrafish (Yao and Ge, 2010; Yao et al., 2014). Kitlga is expressed in the trunk of the body in zebrafish during melanocyte migration stage and later in the skin, and its receptor (kita) is required for melanocyte survival (Hultman et al., 2007). Kitlga and kita genes are also expressed in the ovarian somatic follicle cells and are responsible for oocyte maturation. Since the target tissue of endocrine hormones for regulation of folliculogenesis is somatic follicle cells, the expression of Kitlga in somatic cells possesses a function as an external stimulating factor on IGF-I mediator in PI3K-Akt 
pathway in the gonad (Yao et al., 2014). Kit system genes in mouse testis play an important role in signalling cascades initiated by Kit in PGCs, spermatogenesis and oogenesis. In mouse testis, kit ligand and its receptor is expressed in sertoli cells during spermatogonial development and in leydig cells, which have considerable influence on the endocrine function in mouse spermatogenesis (Kissel et al., 2000). Interestingly, in this study we found the upregulation of kit genes in the male gonads compared to female gonads, which emphasizes the importance of the kit system genes in the spermatogenesis process in zebrafish. To the best of our knowledge, we are the first to report the expression of a series of CP genes in the zebrafish reproduction system, but its underlying biological reason is still unknown and deserves further research.

Contrary to the gonads, no significantly differentially expressed $\mathrm{CP}$ genes were observed in the caudal fin. However, some of those genes showed a high level of expression in both temperature groups. For CP formation in zebrafish, chromatophores arise directly from neural crest cells during embryonic development and later during metamorphosis from stem cells to generate the adult pigment pattern through interactions between different chromatophore cell types (Singh and Nüsslein-Volhard, 2015; Nüsslein-Volhard and Singh, 2017; Patterson et al., 2013; Frohnhöfer et al., 2013). Besides this cell-cell interaction mechanism, agoutisignalling peptide (ASIP) has been observed to control the dorso-ventral patterning in the skin of adult zebrafish (60 and 210 days post fertilization: dpf), resulting in the graded expression of melanin synthesis-involved genes such as mitfa, tyrplb and dct. However, ASIP was not detected to contribute to the pigmentation of the adult fins (Ceinos et al., 2015) due to different mechanisms of stripe formation in the body and fins (Singh and NüssleinVolhard, 2015; Nüsslein-Volhard and Singh, 2017). Nevertheless, the studies of caudal fin regeneration have been shown that the regeneration cells are able to remember their former locations and patterned the caudal fin tissue after injury in adult zebrafish. The expression activity of the kitlga gene is demonstrated to promote the recovery of melanocytes during the regeneration of adult zebrafish caudal fin ( $\mathrm{Tu}$ and Johnson, 2011; Tryon et al., 2014; Rabinowitz et al., 2017). Sex-biased gene study in the tail of guppy has revealed that several male-biased genes encoded proteins with pigment biosynthesis functions (e.g. kita, kitb, mitfa, mitfb, tyrplb, dct and $x d h$ ) (Sharma et al., 2014). In adult zebrafish, sexual dimorphism is illustrated by a brighter yellow colouration in males than in females (Nüsslein-Volhard and Singh, 2017). Therefore, assuming that the differential expression of CP genes may be important for sex-dependent colour patterning during early stages of development, in adults; 
at least their expression levels are more constant. The observed differences in colouration between males and females could be due to post-transcriptional regulation of key enzymes involved in pigment synthesis and distribution. In GSEA, the tight junction and gap junction pathways has been enriched in this study, in which the genes involved in these pathways have a function in $\mathrm{CP}$ development in zebrafish, as their expression was observed in our transcriptome profiles. Interestingly, tuba4 and tuba7l, which play a role in SD in zebrafish, were differentially expressed in these pathways, but their role in $\mathrm{CP}$ of zebrafish needs further investigation. We also observed a few SD genes (e.g. cyp19a1b, zp3b, tekt1, dmrt1 and sycp3) are differentially expressed in the MCF vs. FCF and MTF vs. FTF. Taking into account the fact that the correlations between the expression level of these genes in the gonads and in the caudal fins are relatively weak, the genetic cause of these gene expressions requires further research.

\section{Conclusions}

Elevated water temperature during embryogenesis resulted in male-biased sex ratio in this studied zebrafish population, which supports the hypothesis of a PSD system in domesticated strains. In this study, transcriptome analysis of gonads revealed the activation of pro-male gene expression and repression of pro-female gene expression in male compared to female gonads, leading to gonadal masculinization in laboratory zebrafish. However, unexpected differential expression patterns of the most CP genes were observed in the gonad, suggesting the neofunctionalisation of those genes in zebrafish reproduction system. Contrary to the gonad, the different colouration in the caudal fin of adult fish was not due to the differential expression of $\mathrm{CP}$ genes, even though a high expression magnitude of those genes was observed in both sexes. The observed differences in colouration between males and females may be due to a post-transcriptional regulation of key enzymes involved in pigment synthesis and distribution. Furthermore, we identified a subset of enriched pathways (tight junction, gap junction and apoptosis) containing both SD and CP genes, which may play a pivotal role in regulation of phenotypic sexual dimorphism, regarding the differences in $\mathrm{CP}$ of two sexes in adult zebrafish. 


\section{Methods}

\section{Fish stocks and husbandry}

The Singapore strain of zebrafish was used in this study. This strain was directly imported from a breeding farm in Singapore in 1990 by the Company Aquafarm Ryba Zeven, GmBH (Zeven, Germany) (Sondermann, 1990; Von Hertell et al., 1990) and kept in the aquaculture facilities of the University of Goettingen for research purposes in accordance with approved institutional guidelines. The zebrafish population was kept in mixed sex groups at $28 \pm 0.5^{\circ} \mathrm{C}$. The photoperiod regime was applied 12-h light/12-h dark per day. The fish were fed two times a day with commercial food (Tetramine junior, Germany) and freshly hatched Artemia salina nauplii.

\section{Temperature treatments}

In this study, we used the fertilized eggs derived from full-sib family in equal proportions in order to investigate the effect of high water temperature on sexual plasticity and sex related colour patterning. For this proposed, two temperature treatments were designed: 1) in the first treatment, the animals were kept at the constant temperature of $28^{\circ} \mathrm{C}$ throughout the experiment (control group), and 2) in the second treatment, the eggs were exposed to the high water temperature of $35^{\circ} \mathrm{C}$ (IPCC, 2013; Jyväsjärvi et al., 2015; Brown et al., 2015; Habary et al., 2017) during embryogenesis from 5-24 hpf (treatment group). This development time is a critical phase of embryonic development known as segmentation stage (between gastrula to pharyngula period) (Kimmel et al., 1995; Abozaid et al., 2011; Hosseini et al., 2019). After treatment, the heat-exposed groups were returned to the control temperature at $28^{\circ} \mathrm{C}$. All experimental groups were then kept under the same environmental conditions until sexual maturity. In order to avoid the heat stress, the temperature of treatment group was gradually increased and/or decreased to the target temperature in this study. To ensure the accuracy of the experiment, the temperature of the experimental groups was controlled daily throughout the study. Both temperature treatment and control groups were mixed separately in 36-liter tanks (AquaBox ${ }^{\circledR}$ by Aqua Schwarz GmbH, Goettingen, Germany) two weeks after the eggs had hatched until sexual maturity to eliminate the effects of population density within tanks. After sexual maturity, the urogenital papilla was examined and, in the unclear case, the microscopic examination was performed to determine the sex of each individual. In this 
study, the sex of a total number of 559 individuals was determined in all experimental groups. All husbandry facilities, fish management and water quality control, animal care and feeding are described by details in Hosseini et al. (2019).

\section{Tissue sample collection}

Since the distinct phenotypic sexual dimorphism in colouration between males and females was observed in the caudal fin, the tissue samples of caudal fins and gonads of adult zebrafish were collected for transcriptome analysis. Tissue samples of 6 individuals in each experimental group (control male, control female, treatment male, and treatment female) from two different tissues (gonad and caudal fin) were collected after sexual maturity.

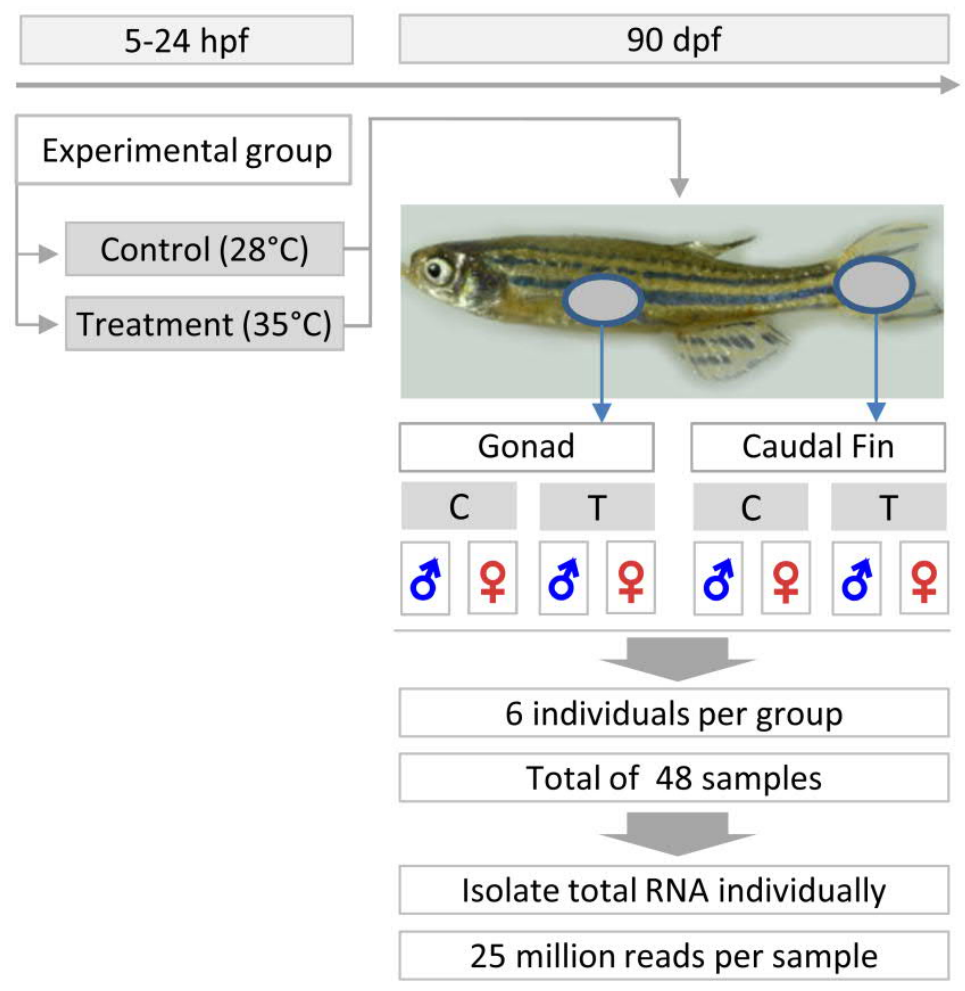

Figure 8: General overview of experimental design and tissue sample (Gonad and Caudal fin) collection for RNA_Seq analysis in this study. Total RNA was isolated from adult zebrafish gonad and caudal fin tissues of each individual separately and used for transcriptome analysis. hpf: hours post fertilization; dpf: days post fertilization; C: control; T: temperature treatment.

A total number of 48 tissue samples (24 caudal fin and 24 gonad samples) were used for transcriptomic analysis (Figure 8). For this purpose, the animals were sacrificed and tissues 
were carefully dissected. Tissue samples were stored in RNA stabilization solution, RNAlater ${ }^{\circledR}$ Tissue Collection (Thermo Fisher Scientific, Germany) and kept at $-20^{\circ} \mathrm{C}$ until starting the molecular laboratory genetic analysis.

\section{RNA extraction and sequencing}

Total RNA of the gonad tissues was isolated using a RNeasy Plus Mini kit (Qiagen, Hilden, Germany) and total RNA from the caudal fin samples was extracted by a RNeasy Fibrous Tissue kit (Qiagen, Hilden, Germany) according to the manufacturer's protocol. The RNA quantity and quality was measured using a Qubit 2.0 Fluorometer (Thermo Fisher Scientific, Waltham, USA) and RNA Screen Tape on an Agilent Bioanalyzer 2100 (Agilent, Santa Clara, USA). The RNA integrity number (RIN) of the most samples was $>7$, exception of two samples with RIN scores of 6.1 and 5.6. The sample libraries preparation was performed from $500 \mathrm{ng}$ input total RNA using the TruSeq stranded mRNA kit, and sequenced on an Illumina HiSeq4000 platform aiming for 25 million 2x75 bp paired-end reads per sample.

\section{RNA read alignment and gene counting}

Quality assessment of raw sequencing data was conducted using FastQC (version 0.11.4) (Andrews, 2010) and MultiQC (version 1.3) (Ewels et al., 2016) for forward and reverse reads. Due to the overall high quality of the reads, no samples had to be excluded from the analysis. FASTQ files were processed with Trimmomatic (version 0.36) (Bolger et al., 2014) to remove the low quality bases and Illumina adapters. The average percentage of paired reads that survived in trimming was $93.5 \% \pm 0.5 \%$. RNA read alignments were then mapped to the Danio rerio genome assembly version GRCz10 (GCA_000002035.3), which was downloaded from Ensembl (Flicek et al., 2014) using STAR-2pass (version 2.4.2a) (Dobin et al., 2013) and resulted in an average mapping success rate of $90.1 \% \pm 3.3 \%$. Finally, FeatureCounts (version 1.4.3) (Liao et al., 2014) was utilized to count the number of reads mapped for each gene with an average successful read assignment of $73.6 \% \pm 4.9 \%$. All aforementioned software packages were run using the standard settings. 


\section{Differential gene expression and gene set enrichment analysis}

All RNA_Seq analyses in this study were performed in the R-Statistics program (R Core Team, 2015) using the "edgeR" package (Robinson et al., 2010). For each tissue (gonad and caudal fin), we conducted four comparisons - (1) males vs. females within treatment and control groups, and (2) treatment vs. control within males and females- to assess the effect of sex and temperature treatment, respectively. This resulted in 8 comparisons, for which we performed a differential gene expression analysis (DGEA).

To investigate the significantly differentially expressed SD and CP genes, we prepared two gene lists for candidate genes: (1) a list containing genes that have been associated with SD; and (2) that have been associated with $\mathrm{CP}$, which are addressed in the different literatures and NCBI gene database for zebrafish. For selection and classification of $\mathrm{CP}$ genes, we used coat colour categories (http://www.espcr.org/micemut) (Braasch et al., 2009) and additional information given by zebrafish database (ZFIN; http://zfin.org) (Ruzicka et al., 2015). A total number of $79 \mathrm{SD}$ and $213 \mathrm{CP}$ genes were used as selected candidate genes in this study (Additional file 2).

For the DGEA, we only considered genes with more than 1 CPM to avoid unreliable results across all samples, of at least one of the eight experimental groups: male control gonad (MCG), female control gonad (FCG), male treatment gonad (MTG), female treatment gonad (FTG), male control fin (MCF), female control fin (FCF), male treatment fin (MTF), and female treatment fin (FTF) to be expressed and use them for further analyses. This resulted in a matrix of 18,871 genes for 48 samples (6 biological replicates per experimental group). The DGEA was conducted using a negative binomial model and the exact test in edgeR. In general, each DGEA was comprised of three main steps: (1) normalization of gene counts for sample-specific effects (sequencing depth and RNA composition); (2) fitting a negative binomial model to the count data to estimate relevant dispersion parameters; and (3) performing an exact test for the negative binomial distribution to compare whether gene expressions are significantly different between two conditions.

To account for multiple testing, we employed the false discovery rate (FDR) approach by Benjamini and Hochberg (Benjamini and Hochberg, 1995). We then compared the results of the 8 comparisons and obtained three sets of significant DEGs (FDR $<0.005, \log _{2} \mathrm{FC}>2$ ), for which we performed a GSEA to gain insight into their biological interpretations. To this end, we first created a pathway and Gene Ontology (GO) annotation based on the Kyoto 
Encyclopedia of Genes and Genomes (KEGG) database (Kanehisa et al., 2012) and GO database (Gene Ontology Consortium, 2015), respectively. Then, for each pathway and GO and each set of significant genes, we performed a Fisher's Exact test to identify pathways and GOs that are enriched with significant genes. Hereby, we considered pathways or GOs that contain at least one significant gene. Due to the small number of animals and since the Fisher's Exact test is known to be conservative, we are aware that our test might be under powered. Therefore, we considered GOs that are highly ranked (the first 5 GOs with the smallest p-values) and in addition 3 other important GOs regarding our objectives in this study as potential candidates for discussion. The GOs and pathways with the p-values less than 0.05 were investigated to be enriched in DGEA. In addition, GOs and pathways with corrected p-values after multiple test correction (Benjamini-Hochberg method) were considered to be significantly enriched in the DGEA and are presented in Additional file 3.

\section{Statistical analysis}

Since sex is a binary variable characterized by 0 and 1 values, and its consideration as the independent variable in a statistical model does not represent the assumption of a normal distribution, and a statistically appropriate function to describe this association is the logistic model, therefore, a linear logistic model was used to investigate the effect of temperature treatment on sex determination. In this case, the dependent variable $\left(\mathrm{y}_{\mathrm{i}}\right)$ represents the value 1 for the probability to be male $\left(\pi_{\mathrm{i}}\right)$ or 0 for the probability to be female $\left(1-\pi_{\mathrm{i}}\right)$ for the observation i.

The logit link function (McCullagh and Nelder, 1983) is defined by Logit $\left(\frac{\pi_{\mathrm{i}}}{1-\pi_{\mathrm{i}}}\right)=\eta_{\mathrm{i}}$ where $\eta_{\mathrm{i}}$ is the probability of being male on the logit scale.

The GLIMMIX procedure of SAS version 9.3 (SAS Institute, 2013) was then used to analyse the data according to the following model: $\eta_{\mathrm{i}}=\mu+\alpha_{\mathrm{i}}$

where $\pi_{\mathrm{i}}$ is the probability of being male, $\mu$ is the general mean effect, $\alpha_{\mathrm{i}}$ is the fixed effect of temperature treatment $\left(\mathrm{i}=1\right.$ : temperature-treated eggs $35^{\circ} \mathrm{C}, \mathrm{i}=2$ : control group $\left.28^{\circ} \mathrm{C}\right)$. Least squares means were estimated on the logit scale and then back transformed using the inverse link function to the original scale (probability to be male) (Littell et al., 2006). 


\section{Additional files}

Additional file 1: Table S1. Differentially expressed genes in male treatment gonad vs. male control gonad (MTG vs. MCG)

Additional file 1: Table S2. Differentially expressed genes in female treatment gonad vs. female control gonad (FTG vs. FCG)

Additional file 1: Table S3. Differentially expressed genes in male treatment fin vs. male control fin (MTF vs. MCF)

Additional file 1: Table S4. Differentially expressed genes in female treatment fin vs. female control fin (FTF vs. FCF)

Additional file 1: Table S5. Differentially expressed genes in male control gonad vs. female control gonad (MCG vs. FCG)

Additional file 1: Table S6. Differentially expressed genes in male treatment gonad vs. female treatment gonad (MTG vs. FTG)

Additional file 1: Table S7. Differentially expressed genes in male control fin vs. female control fin (MCF vs. FCF)

Additional file 1: Table S8. Differentially expressed genes in male treatment fin vs. female treatment fin (MTF vs. FTF)

Additional file 2: Table S9. Selected candidate sex determination genes used in this study

Additional file 2: Table S10. Selected candidate colour patterning genes used in this study

Additional file 2: Table S11. Quantile distribution of colour patterning genes in the caudal fin in the transcriptome profile ( $\log _{2} \mathrm{CPM}$ per mega base pairs)

Additional file 3: Table S12. List of differentially expressed genes in male treatment fin vs. female treatment fin (MTF vs. FTF), which are used for gene set enrichment analysis (GSEA) Additional file 3: Table S13. List of differentially expressed genes in male treatment gonad vs. female treatment gonad (MTG vs. FTG), which are used for gene set enrichment analysis (GSEA)

Additional file 3: Table S14. List of differentially expressed genes in overlapping between comparison groups, which are used for gene set enrichment analysis (GSEA)

Additional file 3: Table S15. Enriched pathways in male treatment fin vs. female treatment fin (MTF vs. FTF)

Additional file 3: Table S16. Enriched pathways in male treatment gonad vs. female treatment gonad (MTG vs. FTG) 
Additional file 3: Table S17. Enriched pathways in overlapping between comparison groups Additional file 3: Table S18. Enriched GOs in male treatment fin vs. female treatment fin (MTF vs. FTF)

Additional file 3: Table S19. Enriched GOs in male treatment gonad vs. female treatment gonad (MTG vs. FTG)

Additional file 3: Table S20. Enriched GOs in overlapping between comparison groups

\begin{abstract}
Abbreviations
SD: sex determination; CP: colour pattern; PSD: polygenic sex determination; PGCs: primordial germ cells; G×E: genotype $\times$ environment interaction; RNA-Seq: RNA sequencing; MCG: male control gonad; FCG: female control gonad; MTG: male treatment gonad; FTG: female treatment gonad; MCF: male control fin; FCF: female control fin; MTF: male treatment fin; FTF: female treatment fin; DEGs: differentially expressed genes; FDR: false discovery rate; FC: fold change; CPM: counts per million reads; Mb: mega base pairs; ASIP: agouti-signalling peptide; dpf: days post fertilization; hpf: hours post fertilization; AGP: adrenal-gonadal primordium; GO: Gene Ontology; KEGG: Kyoto Encyclopedia of Genes and Genomes; GSEA: gene set enrichment analysis; DGEA: differential gene expression analysis.
\end{abstract}

\title{
Acknowledgements
}

We appreciate Dr. Uwe Irion from the Max Planck Institute for Developmental Biology, Tuebingen, Germany for reading and revising the manuscript as well as for his valuable comments on the manuscript.

\section{Funding}

For this research work no funding was received.

\section{Availability of data and materials}

All relevant datasets generated in this study are included within the article and its Additional files. The datasets used and analysed during the current study are available from the corresponding author on reasonable request. 


\section{Author contributions}

SHo contributed to the conception and design of the study, carried out the experiments, molecular genetic lab analysis, interpreted the results and wrote the manuscript. ARS substantially designed the study and conception. ARS and SHo performed computational data analysis and interpreted the data. AF performed RNA-Sequencing. CFG and SHo performed RNA-sequencing data bioinformatics. NTH, SHo and ARS carried out the RNA-sequencing data analysis. GHS provides zebrafish facilities. ARS, HS, SHe, BB and JT edited and corrected the manuscript. All authors read and commented on the manuscript and approved the final version.

\section{Ethics approval}

This study was performed in accordance with the German Animal Protection Law based on national and international recommendations and confirmed by the Animal Welfare Committee of the University of Goettingen (File number E3-17).

\section{Consent for publication}

Not applicable.

\section{Competing interests}

The authors declare that they have no competing interests.

\section{References}

Abozaid H, Wessels S, Hörstgen-Schwark G. Effect of rearing temperatures during embryonic development on the phenotypic sex in zebrafish (Danio rerio). Sex Dev. $2011 ; 5: 259-265$.

Anderson JL, Rodríguez Marí A, Braasch I, Amores A, Hohenlohe P, Batzel P, Postlethwait JH. Multiple sex-associated regions and a putative sex chromosome in zebrafish revealed by RAD mapping and population genomics. PLoS One. 2012; 7:e40701. doi: 10.1371/journal.pone.0040701.

Andrews, S. FastQC: A quality control tool for high throughput sequence data. 2010. http://www.bioinformatics.babraham.ac.uk/projects/ fastqc. 
Baroiller JF, D'Cotta H, Saillant E. Environmental effects on fish sex determination and differentiation. Sex Dev. 2009; 3:118-135.

Benjamini Y, Hochberg Y. Controlling the false discovery rate: A practical and powerful approach to multiple testing. J R Stat Soc B. 1995; 57:289-300.

Bensimon-Brito A, Cardeira J, Cancela ML, Huysseune A, Witten PE. Distinct patterns of notochord mineralization in zebrafish coincide with the localization of Osteocalcin isoform 1 during early vertebral centra formation. BMC Dev Biol. 2012; 12:28.

Betrán E, Thornton K, Long M. Retroposed new genes out of the X in Drosophila. Genome Res. 2002; 12:1854-1859.

Bolger AM, Lohse M, Usadel B. Trimmomatic: A flexible trimmer for Illumina sequence data. Bioinformatics. 2014; 30:2114-20.

Bollig F, Mehringer R, Perner B, Hartung C, Schäfer M, Schartl M, Volff JN, Winkler C, Englert C. Identification and comparative expression analysis of a second wtl gene in zebrafish. Dev Dyn. 2006; 235:554-61.

Braasch I, Brunet F, Volff JN, Schartl M. Pigmentation pathway evolution after WholeGenome duplication in Fish. Genome Biol Evol. 2009; 1:479-493.

Brown AR, Owen SF, Peters J, Zhang Y, Soffker M, Paull GC, Hosken DJ, Wahab MA, Tyler CR. Climate change and pollution speed declines in zebrafish populations. Proc Natl Acad Sci U S A. 2015; 112: E1237-E1246.

Bryson-Richardson RJ, Daggett DF, Cortes F, Neyt C, Keenan DG, Currie PD. Myosin heavy chain expression in zebrafish and slow muscle composition. Dev Dyn. 2005; 233:101822.

Carvalho FR, Fernandes AR, Cancela ML, Gavaia PJ. Improved regeneration and de novo bone formation in a diabetic zebrafish model treated with paricalcitol and cinacalcet. Wound Repair Regen. 2017; 25:432-442.

Ceinos RM, Guillot R, Kelsh RN, Cerdá-Reverter JM, Rotllant J. Pigment patterns in adult fish result from superimposition of two largely independent pigmentation mechanisms. Pigment Cell Melanoma Res. 2015; 28:196-209.

Chou CW, Lin J, Jiang YJ, Liu YW. Aberrant Global and Jagged-Mediated Notch Signaling Disrupts Segregation Between wt1-Expressing and Steroidogenic Tissues in Zebrafish. Endocrinology. 2017; 158:4206-4217.

Conant GC, Wolfe KH. Turning a hobby into a job: How duplicated genes find new functions. Nat Rev Genet. 2008; 9:938-50. 
Cutting A, Chue J, Smith CA. Just how conserved is vertebrate sex determination? Dev Dyn. $2013 ; 242: 380-387$.

Dapper AL, Wade MJ. The evolution of sperm competition genes: the effect of mating system on levels of genetic variation within and between species. Evolution. 2016; 70:502-511.

Devlin RH, Nagahama Y. Sex determination and sex differentiation in fish: An overview of genetic, physiological, and environmental influences. Aquaculture. 2002; 208:191-364.

Díaz N, Piferrer F. Lasting effects of early exposure to temperature on the gonadal transcriptome at the time of sex differentiation in the European sea bass, a fish with mixed genetic and environmental sex determination. BMC Genomics. 2015; 16:679.

Dobin A, Davis CA, Schlesinger F, Drenkow J, Zaleski C, Jha S, Batut P, Chaisson M, Gingeras TR. STAR: Ultrafast universal RNA-seq aligner. Bioinformatics. 2013; 29:15-21.

Dooley CM, Mongera A, Walderich B, Nüsslein-Volhard C. On the embryonic origin of adult melanophores: the role of ErbB and Kit signalling in establishing melanophore stem cells in zebrafish. Development. 2013; 140:1003-13.

Dranow DB, Hu K, Bird AM, Lawry ST, Adams MT, Sanchez A, Amatruda JF, Draper BW. Bmp15 is an Oocyte-produced signal required for maintenance of the adult female sexual phenotype in Zebrafish. PLoS Genet. 2016; 12:e1006323.

Elicker KS, Hutson LD. Genome-wide analysis and expression profiling of the small heat shock proteins in zebrafish. Gene. 2007; 403:60-9.

Ellegren H, Parsch J. The evolution of sex-biased genes and sex-biased gene expression. Nat Rev Genet. 2007; 8:689-698.

Ewels P, Magnusson M, Lundin S, Käller M. MultiQC: summarize analysis results for multiple tools and samples in a single report. Bioinformatics. 2016; 32:3047-8.

Fadeev A, Krauss J, Frohnhöfer HG, Irion U, Nüsslein-Volhard C, et al. Tight Junction Protein 1a regulates pigment cell organisation during zebrafish colour patterning. Elife. 2015; 4:e06545.

Fernandino JI, Hattori RS, Kimura H, Strüssmann CA, Somoza GM. Expression profile and estrogenic regulation of anti-Müllerian hormone during gonadal development in pejerrey Odontesthes bonariensis, a teleost fish with strong temperature-dependent sex determination. Dev Dyn. 2008; 237:3192-3199.

Flicek P, Amode MR, Barrell D, Beal K, Billis K, Brent S, Carvalho-Silva D, Clapham P, Coates G, Fitzgerald S, Gil L, Girón CG, Gordon L, Hourlier T, Hunt S, Johnson N, 
Juettemann T, Kähäri AK, Keenan S, Kulesha E, Martin FJ, Maurel T, McLaren WM, Murphy DN, Nag R, Overduin B, Pignatelli M, Pritchard B, Pritchard E, Riat HS, Ruffier M, Sheppard D, Taylor K, Thormann A, Trevanion SJ, Vullo A, Wilder SP, Wilson M, Zadissa A, Aken BL, Birney E, Cunningham F, Harrow J, Herrero J, Hubbard TJ, Kinsella R, Muffato M, Parker A, Spudich G, Yates A, Zerbino DR, Searle SM. Ensembl 2014. Nucleic Acids Res. 2014; 42:D749-55.

Frohnhöfer HG, Krauss J, Maischein HM, Nüsslein-Volhard C. Iridophores and their interactions with other chromatophores are required for stripe formation in zebrafish. Development. 2013; 140:2997-3007.

Gene Ontology Consortium: Going forward. Nucleic Acids Res. 2015; 43:D1049-56.

Habary A, Johansen JL, Nay TJ, Steffensen JF, Rummer JL. Adapt, move or die - how will tropical coral reef fishes cope with ocean warming? Glob Chang Biol. 2017; 23:566577.

Hattori RS, Gould RJ, Fujioka T, Saito T, Kurita J, Strüssmann CA, Yokota M, Watanabe S. Temperature-dependent sex determination in Hd-rR Medaka Oryzias latipes: gender sensitivity, thermal threshold, critical period, and DMRT1 expression profile. Sex Dev. 2007; 1:138-46.

Heinen TJ, Staubach F, Häming D, Tautz D. Emergence of a new gene from an intergenic region. Curr Biol. 2009; 19:1527-1531.

Hosseini S, Brenig B, Tetens J, Sharifi AR. Phenotypic plasticity induced using high ambient temperature during embryogenesis in domesticated zebrafish, Danio rerio. Reprod Domest Anim. 2019; 54:435-444.

Hosseini S, Herzog S, Ha NT, Falker-Gieske C, Brenig B, Tetens J, Simianer H, Sharifi AR. Transcriptome analysis and classifications of sex using neural network in domesticated zebrafish. EAAP - 69th Annual Meeting, Book of abstracts No. 24. Dubrovnik, Croatia. Wageningen academic publishers, The Netherlands; 2018. P. 297.

Hsu HJ, Lin G, Chung BC. Parallel early development of zebrafish interrenal glands and pronephros: differential control by wt1 and ff1b. Development. 2003; 130:2107-16.

Hultman KA, Bahary N, Zon LI, Johnson SL. Gene duplication of the zebrafish kit ligand and partitioning of melanocyte development functions to kit ligand a. PLoS Genet. 2007; 3:e17.

IPCC Climate Change 2013: The Physical Science Basis. Working Group I Contribution to the Fift Assessment Report of the Intergovernmental Panel on Climate Change. 2013, Cambridge University Press, Cambridge, UK. 
Jyväsjärvi J, Marttila H, Rossi PM, Ala-Aho P, Olofsson B, Nisell J, Backman B, Ilmonen J, Virtanen R, Paasivirta L, Britschgi R, Kløve B, Muotka T. Climate-induced warming imposes a threat to north European spring ecosystems. Glob Chang Biol. 2015; 21:4561-9.

Kaessmann, H. Origins, evolution, and phenotypic impact of new genes. Genome Res. 2010; 20:1313-26.

Kanehisa M, Goto S, Sato Y, Furumichi M, Tanabe M. KEGG for integration and interpretation of large-scale molecular data sets. Nucleic Acids Res. 2012; 40:D109-14.

Kimmel CB, Ballard WW, Kimmel SR, Ullmann B, Schilling TF. Stages of embryonic development of the zebrafish. Dev Dyn. 1995; 203:253-310.

Kissel H, Timokhina I, Hardy MP, Rothschild G, Tajima Y, Soares V, Angeles M, Whitlow SR, Manova K, Besmer P. Point mutation in kit receptor tyrosine kinase reveals essential roles for kit signaling in spermatogenesis and oogenesis without affecting other kit responses. EMBO J. 2000; 19:1312-26.

Klüver N, Herpin A, Braasch I, Driessle J, Schartl M. Regulatory back-up circuit of medaka Wt1 co-orthologs ensures PGC maintenance. Dev Biol. 2009; 325:179-88.

Klüver N, Yang L, Busch W, Scheffler K, Renner P, Strähle U, Scholz S. Transcriptional response of zebrafish embryos exposed to neurotoxic compounds reveals a muscle activity dependent hspb11 expression. PLoS One. 2011; 6:e29063.

Knoll-Gellida A, André M, Gattegno T, Forgue J, Admon A, Babin PJ. Molecular phenotype of zebrafish ovarian follicle by serial analysis of gene expression and proteomic profiling, and comparison with the transcriptomes of other animals. BMC Genomics. 2006; 7:46.

Kobayashi Y, Nagahama Y, Nakamura M. Diversity and plasticity of sex determination and differentiation in fishes. Sex Dev. 2013; 7:115-125.

Lee SLJ, Horsfield JA, Black MA, Rutherford K, Fisher A, Gemmell NJ. Histological and transcriptomic effects of $17 \alpha$-methyltestosterone on zebrafish gonad development. BMC Genomics. 2017; 18:557.

Liao Y, Smyth GK, Shi W. featureCounts: An efficient general purpose program for assigning sequence reads to genomic features. Bioinformatics. 2014; 30:923-30.

Liew WC, Bartfai R, Lim Z, Sreenivasan R, Siegfried KR, Orban L. Polygenic sex determination system in zebrafish. PLoS One. 2012; 7:e34397.

Liew WC, Orbán L. Zebrafish sex: A complicated affair. Brief. Funct. Genomics. 2014; 13:172-187. 
Littell RC, Milliken GA, Stroup WW, Wolfinger RD, Schabenberger O. SAS for Mixed Models. SAS Inst., Cary, NC; 2006.

Liu W, Li SZ, Li Z, Wang Y, Li XY, Zhong JX, Zhang XJ, Zhang J, Zhou L, Gui JF. Complete depletion of primordial germ cells in an All-female fish leads to Sex-biased gene expression alteration and sterile All-male occurrence. BMC Genomics. 2015; 16:971.

Mahalwar P, Singh AP, Fadeev A, Nüsslein-Volhard C, Irion U. Heterotypic interactions regulate cell shape and density during colour pattern formation in zebrafish. Biol Open. 2016; 5:1680-1690.

Mahalwar P, Walderich B, Singh AP, Nüsslein-Volhard C. Local reorganization of xanthophores fine-tunes and colours the striped pattern of zebrafish. Science. 2014; 345:1362-4.

Marvin M, O'Rourke D, Kurihara T, Juliano CE, Harrison KL, Hutson LD. Developmental expression patterns of the zebrafish small heat shock proteins. Dev Dyn. 2008; 237:454-63.

McCullagh P, Nelder JA. Generalized Linear Models. Chapman \& Hall, London, UK; 1983.

Mold DE, Dinitz AE, Sambandan DR. Regulation of zebrafish zona pellucida gene Activity in developing oocytes. Biol Reprod. 2009; 81:101-10.

Nakamura S, Kobayashi D, Aoki Y, Yokoi H, Ebe Y, Wittbrodt J, Tanaka M. Identification and lineage tracing of two populations of somatic gonadal precursors in medaka embryos. Dev Biol. 2006; 295:678-88.

Nüsslein-Volhard C, Singh AP. How fish colour their skin: A paradigm for development and evolution of adult patterns. Bioessays. 2017; 39:3.

Ospina-Alvarez N, Piferrer F. Temperature-dependent sex determination in fish revisited: prevalence, a single sex ratio response pattern, and possible effects of climate change. PLoS One. 2008; 3:e2837.

Patterson LB, Parichy DM. Interactions with iridophores and the tissue environment required for patterning melanophores and xanthophores during zebrafish adult pigment stripe formation. PLoS Genet. 2013; 9:e1003561.

Perner B, Englert C, Bollig F. The Wilms tumor genes $w t 1 a$ and $w t 1 b$ control different steps during formation of the zebrafish pronephros. Dev Biol. 2007; 309:87-96.

Poonlaphdecha S, Pepey E, Canonne M, de Verdal H, Baroiller JF, D'Cotta H. Temperature induced-masculinization in the Nile tilapia causes rapid up-regulation of both dmrt1 and amh expressions. Gen Comp Endocrinol. 2013; 193:234-242. 
$\mathrm{R}$ Core Team. R: a language and environment for statistical computing. Vienna: $\mathrm{R}$ Foundation for Statistical Computing; 2015. https://www.r-project.org/

Rabinowitz JS, Robitaille AM, Wang Y, Ray CA, Thummel R, Gu H, Djukovic D, Raftery D, Berndt JD, Moon RT. Transcriptomic, proteomic, and metabolomic landscape of positional memory in the caudal fin of zebrafish. Proc Natl Acad Sci U S A. 2017; 114:E717-E726.

Ribas L, Liew WC, Díaz N, Sreenivasan R, Orbán L, Piferrer F. Heat-induced masculinization in domesticated zebrafish is family-specific and yields a set of different gonadal transcriptomes. Proc Natl Acad Sci U S A. 2017; 114:E941-E950.

Richardson BE, Lehmann R. Mechanisms guiding primordial germ cell migration: strategies from different organisms. Nature Reviews Molecular Cell Biology. 2010; 11:37-49.

Robinson MD, McCarthy DJ, Smyth GK. edgeR: A Bioconductor package for differential expression analysis of digital gene expression data. Bioinformatics. 2010; 26:139-140.

Rodríguez-Marí A, Wilson C, Titus TA, Canestro C, BreMiller RA, Yan YL, Nanda I, Johnston A, Kanki JP, Gray EM, He X, Spitsbergen J, Schindler D, Postlethwait JH. Roles of brca2 (fancdl) in oocyte nuclear architecture, gametogenesis, gonad tumors, and genome stability in zebrafish. PLoS Genet. 2011; 7:e1001357.

Rougeot C, Prignon C, Ngouana Kengne CV, Mélard C. Effect of high temperature during embryogenesis on the sex differentiation process in the Nile tilapia, Oreochromis niloticus. Aquaculture. 2008; 276:205-208.

Ruzicka L, Bradford YM, Frazer K, Howe DG, Paddock H, Ramachandran S, Singer A, Toro S, Van Slyke CE, Eagle AE, Fashena D, Kalita P, Knight J, Mani P, Martin R, Moxon SA, Pich C, Schaper K, Shao X, Westerfield M. ZFIN, The zebrafish model organism database: Updates and New Directions. Genesis. 2015; 53:498-509.

Santos D, Luzio A, Coimbra AM. Zebrafish sex differentiation and gonad development: A review on the impact of environmental factors. Aquat Toxicol. 2017; 191:141-163.

Santos EM, Workman VL, Paull GC, Filby AL, Van Look KJ, Kille P, Tyler CR. Molecular basis of sex and reproductive status in breeding zebrafish. Physiol Genomics. 2007; 30:111-22.

SAS Institute Inc. 2013.SAS/STAT®13.1 User's Guide. Cary, NC, USA; 2013. https://support.sas.com/documentation/onlinedoc/stat/131/glimmix.pdf

Schnerwitzki D, Perner B, Hoppe B, Pietsch S, Mehringer R, Hänel F, Englert C. Alternative splicing of Wilms tumor suppressor 1 (Wt1) exon 4 results in protein isoforms with different functions. Dev Biol. 2014; 393:24-32. 
Selim KM, Shinomiya A, Otake H, Hamaguchi S, Sakaizumi M. Effects of high temperature on sex differentiation and germ cell population in medaka, Oryzias latipes. Aquaculture. 2009; 289:340-349.

Sharma E, Künstner A, Fraser BA, Zipprich G, Kottler VA, Henz SR, Weigel D, Dreyer C. Transcriptome assemblies for studying sex-biased gene expression in the guppy, Poecilia reticulate. BMC Genomics. 2014; 15:400.

Siegfried KR, Nüsslein-Volhard C. Germ line control of female sex determination in zebrafish. DEV BIOL. 2008; 324:277-287.

Singh AP, Frohnhöfer HG, Irion U, Nüsslein-Volhard C. Response to comment on "Local reorganization of xanthophores fine-tunes and colours the striped pattern of zebrafish. Science. 2015; 348:297.

Singh AP, Nüsslein-Volhard C. Zebrafish Stripes as a Model for Vertebrate Colour Pattern Formation. Curr Biol. 2015; 25: R81-R92.

Small CM, Carney GE, Mo Q, Vannucci M, Jones AG. A microarray analysis of sex- and gonad-biased gene expression in the zebrafish: evidence for masculinization of the transcriptome. BMC Genomics. 2009;10:579.

Sondermann U. Untersuchungen zur Variabilität der Toxizitätsempfindlichkeit des Zebrabärblings (Brachydanio rerio). Dissertation, Department of Animal Sciences, University of Goettingen. Goettingen, Germany; 1990. pp.71.

Stengel F, Baldwin AJ, Painter AJ, Jaya N, Basha E, Kay LE, Vierling E, Robinson CV, Benesch JL. Quaternary dynamics and plasticity underlie small heat shock protein chaperone function. Proc Natl Acad Sci U S A. 2010; 107:2007-12.

Tryon RC, Johnson SL. Clonal analysis of kit ligand a functional expression reveals lineage specific competence to promote melanocyte rescue in the mutant regenerating caudal fin. PLoS One. 2014; 9:e102317.

Tu S, Johnson SL. Fate restriction in the growing and regenerating zebrafish fin. Dev Cell. $2011 ; 20: 725-32$.

Tzung KW, Goto R, Saju JM, Sreenivasan R, Saito T, Arai K, Yamaha E, Hossain MS, Calvert ME, Orbán L. Early depletion of primordial germ cells in zebrafish promotes testis formation. Stem Cell Reports. 2015; 4:61-73.

Volkening A, Sandstede B. Modelling stripe formation in zebrafish: an agent-based approach. J R Soc Interface. 2015; 12:112. 
Von Hertell U, Hörstgen-Schwark G, Langholz HJ. Family studies on genetic variability in growth and reproductive performance between and within test fish populations of the zebrafish (Brachydanio rerio). Aquaculture. 1990; 85:307-315.

Von Hofsten J, Olsson PE. Zebrafish sex determination and differentiation: involvement of FTZ-F1 genes. Reprod Biol Endocrinol. 2005; 3:63.

Walderich B, Singh AP, Mahalwar P, Nüsslein-Volhard C. Homotypic cell competition regulates proliferation and tiling of zebrafish pigment cells during colour pattern formation. Nat Commun. 2016; 7:11462.

Wang XG, Orban L. Anti-Müllerian hormone and 11 beta-hydroxylase show reciprocal expression to that of aromatase in the transforming gonad of zebrafish males. Dev Dyn. 2007; 236:1329-38.

Webster KA, Schach U, Ordaz A, Steinfeld JS, Draper BW, Siegfried KR. Dmrt1 is necessary for male sexual development in zebrafish. DEV BIOL. 2017; 422:33-46.

Wilson CA, High SK, McCluskey BM, Amores A, Yan YL, Titus TA, Anderson JL, Batzel P, Carvan MJ 3rd, Schartl M, Postlethwait JH. Wild sex in zebrafish: Loss of the natural sex determinant in domesticated strains. Genetics. 2014; 198:1291-1308.

Yang L, Zhang Z, He S. Both Male-Biased and Female-Biased Genes Evolve Faster in Fish Genomes. Genome Biol Evol. 2016; 8:3433-3445.

Yao K, Ge W. Kit system in the zebrafish ovary: evidence for functional divergence of two isoforms of kit (kita and kitb) and kit ligand (kitlga and kitlgb) during folliculogenesis. Biol Reprod. 2010; 82:1216-26.

Yao K, Lau SW, Ge W. Differential regulation of kit ligand A expression in the ovary by IGF-I via different pathways. Mol Endocrinol. 2014; 28:138-50. 


\section{CHAPTER 5}

General Discussion 


\section{Overview}

This thesis focused on the influence of high ambient temperature on sexual and phenotypic plasticity and its interaction with the genotype of zebrafish, in order to better understand the consequences of climate change on zebrafish and many other closely related species in nature. These effects were studied from different point of views in the previous chapters. In chapter 2 and $\mathbf{3}$, the physiological and biological responses to high ambient temperature including sex ratio, survival ability, body size, and colour intensity were investigated. In chapter 4, we examined the hypothesis of how the expression profiles of pigmentation genes are responsible for the development of caudal fin colouration in adult fish, leading to sexually phenotypic variations. To address this hypothesis, in chapter 4 the genetic regulation of colour pattern and its association with sexual dimorphism in response to elevated water temperature were investigated. In chapter 5, we will first discuss further consequences of increased ambient temperature in nature and its effect on phenotypic plasticity (study of chapter 2 and 3), and then examine additional analyses performed regarding the genetic mechanism of the interplay of sex and colour pattern genes, which were not conducted in detail in chapter 4. At the end, future perspectives for further research in this context are given.

\section{Effects of climate change and phenotypic plasticity}

Global climate change leads to an increase of temperature in natural water ecosystems, a reduction in the amount of dissolved oxygen, an acidification of water (decrease in $\mathrm{pH}$ ) due to uptake of $\mathrm{CO}_{2}$, a reduction in nutrient availability, a rise in sea level and a change in water currents. As predicted by the Intergovernmental Panel on Climate Change (IPCC), not only the average temperature of natural waters will rise up to $\sim 4^{\circ} \mathrm{C}$ by the end of this century (Solomon et al., 2007; Shen and Wang, 2014), but also the intensity and frequency of its fluctuations will increase (IPCC, 2013). Elevated water temperature will have threatening effects on aquatic species and ecosystems, altering ecological processes and the geographical distribution of marine species (Poff et al., 2002). Aquatic species including marine fishes, seabirds, marine mammals, plankton, corals and penguins are at the high risk of mortalities, reduced reproductive capacity and migration to more suitable habitats in response to elevated temperatures. Global temperature fluctuations have a larger effect on thermosensitive fish 
species such as TSD and GSD + TE than GSD fish species (Ospina-Álvarez and Piferrer, 2008). This is true, as our result showed for a large number of families (69 families) in chapter 2 of this thesis, rising temperature caused mortality not only during embryonic development but its effect was also observed continuously after hatching. We studied the effect of high temperature perturbation during thermosensitive embryonic development period; however, this negative effect may also be present if the high temperature acts during the larval stage.

In addition, sex alteration in individuals and the subsequently unbalanced sex ratio of populations in response to increased water temperature has been clearly observed in this study as discussed in chapter 2, 3 and $\mathbf{4}$ of this thesis. Heat-exposed masculinization is one of the most important effects of high ambient temperature in zebrafish (Abozaid et al., 2011; Ribas et al., 2017) and many closely related species with a similar sex determination mechanism (Baroiller et al., 2009; Poonlaphdecha et al., 2013; Díaz and Piferrer, 2015). The sex ratio influences the reproductive capacity of populations (Piferrer et al., 2012) and the reproductive success in many fish species is determined by the number of eggs produced by females (Shen and Wang, 2014). Therefore, the absence or decrease in the number of females in a population will affect the size and structure of the population. Thermosensitive fish species are not able to adapt rapidly to temperature changes (Shen and Wang, 2014). This leads to the hypothesis that these species are more vulnerable to the risk of extinction in the wild due to global warming (Brown et al., 2014). Researchers have studied how aquatic species cope with the increase water temperature in the wild for quite some time. Various responses to the potential impacts of climate change on aquatic ecosystems have been observed or predicted including migration, malformation, adaptation and non-adaptation. The physiological response to climate change as a stress factor increases the probability of species extinction and loss of biodiversity, especially in freshwater species living in closed ecosystems and shallow waters such as lakes, streams, rivers, wetlands and coastal areas. Previous studies documented that more than 365 tropical coral reef fish species have moved from their natural habitat and attempted to migrate to a still suitable habitat. They found that some of these species will expand their latitudinal ranges up to $26 \mathrm{~km}$ per decade in response to global warming (Feary et al., 2014; Habary et al., 2017). However, other aquatic species that are less mobile such as plankton are affected by thermal changes in this area and their survival depends on their capacity to acclimatize (Habary et al., 2017). Migration to a new habitat has a negative ecological impact on the distribution and communities of native fish 
species and leads to a new combination of native and non-native species, which will interact in the novel situations (Poff et al., 2002; Sharma et al., 2007). It has also been reported that the elevated water temperatures have a strong negative impact on fish populations in cold ecosystems such as northern Canada and the USA, similar to the tropical fish species (Sharma et al., 2007; Jyväsjärvi et al., 2015; Pandit et al., 2017). It has therefore been predicted that the effects of global warming are more pronounced in organisms living in extreme latitudes (i.e. near the equator or poles), which are more sensitive to warming due to the lower temperature variations in these areas and have lower evolutionary capacity to adapt and/or acclimatize (Huey and Kingsolver, 1993; Tewksbury et al., 2008; Lough, 2012; Habary et al., 2017). Additionally, a reduction in body size of species living in higher altitudes and latitudes were reported in response to climate change (Ohlberger, 2013; Messmer et al., 2017). Some studies predicted that the body size of marine fish species would decline to 2050 during global warming (Cheung et al., 2013), while the underlying responsible mechanism is not clear (Messmer et al., 2017). In this thesis, in chapter $\mathbf{2}$ and $\mathbf{3}$, using a short heat shock during embryonal development in zebrafish, we found a higher body size compared to the control group in adult stage. This observation may indicate an increase in expression of growth-related genes, as observed in other species (Serrana et al., 2012). However, if the elevated ambient temperatures persist over a longer period of time, high temperatures may have the opposite effect, particularly in adulthood, and the adaptation mechanisms may lead to a reduction in body size, as expected for marine species during global warming scenario.

\section{Sex determination and quantifying of secondary sexual traits using machine learning approaches}

Machine learning approaches have developed into a powerful and leading technique with high performance for data processes in life sciences, particularly in phenotypic classification of biological images (Jeanray et al., 2015; Angermueller et al., 2016; Liakos et al., 2018). Convolutional Neural Networks (CNNs), a deep learning architecture, recently has surpassed human-level accuracy for most image recognition and classification (LeCun et al., 2015). These methods have allowed us to automate sex classification in adult animals with high accuracy, which is more reliable than traditional methods. In chapter $\mathbf{3}$, we classified the sex of adult fish using two different machine learning methods (Deep Convolutional Neural 
Networks; DCNNs, and Support Vector Machine; SVM), which possess the advantage of automatisation and robustness. The conventional guidelines for phenotypic sex classification in zebrafish were explained: males generally exhibit a pinkish-gold colour, while females have a bluish-white colour and possess a rounder shape compared to the males. However, not all females present the obvious rounder shape with a distended abdomen and in some cases males and females exhibit similar body colouration (McMillan et al., 2015). Hence, these guidelines are not always applicable and are a highly time-consuming method for biologists to determine the sex. Furthermore, on the basis of our experience in this study, the distinct differences between male and female body colourations were visible and appear clearly only in the water, when the animals were swimming. However, the colour pattern of the caudal fin in the zebrafish strain studied here showed an obvious difference between two sexes in both situations - inside and outside of the water. We speculate that the reason for our observation is the absence or presence of iridophore pigment cell types in the trunk of the body and fins. In zebrafish, iridophores, which are distributed in the middle layer of the skin, display a silvery or blue colouration and give the body a shiny apearince by reflecting light. However, other pigment cell types such as yellow xanthophores (in the outermost layer) and black melanophores (in the basal layer) absorb the short-wave light and light across the spectrum, respectively. Studies on zebrafish stripe pattern formations have shown that the stripe formation mechanism in anal and caudal fins differs from the body stripe formation, where iridophores are not involved in the fin strip patterns (Patterson and Parichy, 2013; Frohnhöfer et al., 2013; Singh and Nüsslein-Volhard, 2015). The ambiguities of these guidelines illustrate that the conventional sex determination method in zebrafish is subjective and prone to error in the prediction process. Hence, our findings in chapter $\mathbf{3}$ of this thesis regarding sexually dimorphism of caudal fin colouration using a new methodology for sex determination by image analysis technology will facilitate the differentiation of male and female zebrafish. This method is not only appropriate for zebrafish; a similar approach can also be adopted for other species with phenotypic sexual dimorphism.

In chapter 3 of this thesis, we have also quantified the influences of high water temperature on colour intensity. Using SVM, we have shown that there is a difference between the colour intensity of male and female caudal fins in zebrafish, an important aspect with regard to the sexual attractiveness and mating success, which has not been reported in zebrafish so far. Heat treatment, however, causes a reduction in the colour intensity in some male animals. 
Generally, male zebrafish display a more intense yellow colouration than females, particularly during sexual activity, in which intensity of colour may play an important role for sexual attractiveness of an individual for mating success (Singh and Nüsslein-Volhard, 2015). In addition, the loss of pigmentation in heat-induced zebrafish was observed in one study, where the animals were exposed to elevated water temperature during larval stage (Ribas et al., 2017). Since exposure to high ambient temperature in this species leads to masculinization and the reduced colouration in the caudal fin was observed in a number of heat-treated males, we hypothesise that these animals are probably masculinized animals, which are genotypically females, but exhibits a phenotypic male features. In respect to the theory of sexual selection and the secondary sexual characteristics for reproductive success, we then asked whether there is an association between colouration and body size. Previous studies indicated that female zebrafish prefer larger males for mating and spawning. There is a positive relationship between partner quality and reproductive investment in terms of allocation theory; females distribute their reproductive resources to the larger males to ensure reproductive success (Pyron, 2003; Skinner and Watt, 2007; Uusi-Heikkilä et al., 2012). In this thesis, we found a positive association between caudal fin colouration and body size in male zebrafish, which appear to play a significant role for sexual selection. Therefore, in addition to the various reported or expected effects of high ambient temperature on aquatic animals and the ecosystem, in this thesis we report further effects of elevated water temperature on colour patterning (secondary sexual trait) of zebrafish as a model animal, which in turn may influence mating and reproductive success of marine fish species in nature during global climate change.

\section{The genetic analysis of sex-associated colour patterns}

As previously explained, there is a sexual dimorphism in the caudal fin colouration of males and females. This should be reflected in genetic association between sex determination and colour patterning genes. A further question arises: Does the high ambient temperature affect their expression? To address this question, transcriptome analysis was performed in chapter 4 of this thesis, investigating the expression profiles of sex-biased genes in response to elevated temperature. We found upregulation of pro-male genes and downregulation of profemale genes in male gonads compared to the females resulting in gonadal masculinization in zebrafish. In contrast to the gonad, a significantly differentially expression of colour pattern genes was not observed in the caudal fin of two different sexes in adult fish. Hence, the 
different colouration in the caudal fin of adult fish was not due to the differential expression of colour pattern genes during adulthood, even though a high expression magnitude of those genes was observed in both sexes. Therefore, in chapter $\mathbf{4}$, we came to the conclusion that the reason for the sexual dimorphism in the caudal fin colouration of adult male and female zebrafish is as follows: 1) assuming that the differential expression of colour pattern genes may be important for sex-dependent colour patterning in early developmental stages (during metamorphosis; 3-6 weeks post fertilization, Nüsslein-Volhard and Singh, 2017); at least their expression levels are more constant during adult stages, 2) the observed differences in colouration between males and females could be due to post-transcriptional regulation of key enzymes involved in pigment synthesis and distribution. In order to test the hypothesis, we set up in chapter 4. i. e., whether there is a genetic association between sex determination and colour pattern genes in zebrafish, we have performed additional analyses in chapter $\mathbf{5}$ of this thesis, which we will discuss in the following sections.

\section{Annotated pathways}

To investigate the main question arising from the results of chapter $\mathbf{4}$, as aforementioned, additional pathway analyses were performed. In this context, we used the selected candidate sex determination and colour pattern genes of zebrafish to search the candidate pathways, whose member colour and sex genes were annotated within a pathway in the KEGG database (Kanehisa et al., 2012) using an R-statistics program (R Core Team, 2015). To this end, we identified a subset of 30 unique and non-redundant pathways enriched for sex and colour genes, in which at least one gene of both gene groups was annotated in the same pathway (Table 1). Of these, we interestingly found that three pathways (tight junction, gap junction, and apoptosis) were annotated previously in gene set enrichment analysis in chapter 4 of this thesis. This result confirms the involvement of sex and colour genes in the regulation of phenotypic sexual dimorphism in zebrafish. Among the annotated pathways listed in Table 1, we reference the interesting pathways enriched for the key sex determination and colour pattern genes in this study as potential candidates for discussion. We identified two major developmental pathways, namely p53 signaling pathway (pro-male pathway) and Wnt signaling pathway (pro-female pathway), which play decisive roles in zebrafish gonadal differentiation (Liew and Orban, 2014). In respect to the sex determination mechanism, a shift in the balance of pro-male and pro-female pathways in the developing gonad will determine the sexual fate of an organism. Upregulation of p53 signaling pathway in 
developing gonad leads to apoptosis process and downregulation of Wnt signaling pathway resulting in testicular differentiation (Liew and Orban, 2014). External stimulation such as high temperature can modify the levels of p53-mediated germ cell apoptosis (Rodríguez-Marí et al., 2010), which affect primordial germ cells survival during embryonic development (Liu et al., 2015; Tzung et al., 2015) and/or oocyte apoptosis in the 'juvenile ovary' in larval stage (Uchida et al., 2002) resulting in sex imbalance towards an increased proportion of males in adult zebrafish. Heat-treatment during gonadal differentiation in zebrafish leads to downregulation of the Wnt target genes (e.g. lefl and ctnnbipl) and a reduction in the expression of the gonadal aromatase gene (cyp19ala) due to an increase in the activation of p53 signaling pathway during the ovary-to-testis transformation process (Sreenivasan et al., 2014). In addition to these gonad differentiation pathways, several other pathways and genes are involved in the regulation of sexual fate in developing gonad of zebrafish (Lee et al., 2017; Ribas et al., 2017). Apart from the genes that act in gonad differentiation, these pathways also contain genes involved in pigmentation such as the pteridine synthesis gene, $m y c b$, which is required for xanthophores pigment cells development (Guyader et al., 2005), melanophore developmental genes; wntl, apc, lefl (Braasch et al., 2009) and the genes with systemic effects; fas, casp3a, casp3b.

Furthermore, we identified the Cytokine-cytokine receptor interaction pathway; a pro-male pathway in zebrafish (Ribas et al., 2017), which encompasses the key testis differentiation gene (amh; Wang and Orban, 2007; Webster et al., 2017) and the most important yellow xanthophores associated genes; csflra (Parichy and Turner, 2003; Patterson et al., 2014) in the same pathway. The same holds true for colour pattern pathways such as Melanogenesis pathway, in which the melanophore development genes; kitlga and kitlgb and their receptor; kita and kitb, respectively (Hultman et al., 2007; Dooley et al., 2013) are annotated with the target genes (wnt4a, ctnnbl, lefl) of one of the pro-female pathways (Wnt signaling pathway). Kit family genes are more specialized for melanophore survival, development and migration (Hultman et al., 2007; Dooley et al., 2013), while csflra is essential for maintaining cells of the xanthophore lineage and is the closest homologue of kit known (Parichy and Turner, 2003). Mitfa (Johnson et al., 2011) is another colour pattern gene that regulates the expression of genes in melanophore developmental process such as tyrp $1 b$ and $d c t$ (Ceinos et al., 2015), which is annotated with gonadal apoptosis gene tp53 (Liew and Orban, 2014) in Mitophagy-animal pathway. The important zebrafish pro-male genes are annotated with the xanthophores and pteridine synthesis genes in different pathways: 
Cytokine-cytokine receptor interaction pathway containing amh (sex) and csflra (colour), MAPK signaling pathway containing tp53 (sex) and mycb (colour), TGF-beta signaling pathway containing amh (sex) and mycb (colour). One may assume that the expression of those genes encoding proteins with functions in pigment biosynthesis in a pathway is related to the distribution of intense yellow colouration in males. In adult zebrafish, interactions between chromatophores are required for strip pattern formation (Irion et al., 2014; Singh et al., 2015; Nüsslein-Volhard and Singh, 2017). Communication between xanthophores and melanophores (cell-cell interactions) are mediated by Connexin 41.8 (cx41.8), encoding a component of gap junction in the strip pattern formation on the body of adult fish (Irion et al., 2014; Singh and Nüsslein-Volhard, 2015). In the absence of gap junction channels, dense iridophore regions expand and fully fill the space of the stripes, which is regulated by Tight Junction Protein 1a (tjpla), suggesting that the tjpla may be involved in communication with xanthophores and melanophores (Fadeev et al., 2015). A crosstalk between different pathways may play a role for the generation of the complex strip pattern in adult zebrafish. However, there is still a lack of understanding of the function of these complex pathways and how they interact. In this context, a study of sex-biased gene expression in guppy fish illustrated a higher expression of several pigmentation genes (e.g. kita, kitb, mitfa, mitfb, tyrplb, $d c t$ and $x d h$ ) in males tail tissue compared to females, which demonstrated malebiased colour pattern gene expression (Sharma et al., 2014). Taking together, the enrichment of these pathways, which include sex determination and colour pattern genes in the same pathway as obvious examples provides evidence for the association between sex and colour genes in zebrafish in respect to the sexual dimorphism in adult fish. 
Table 1: KEGG database pathways based on selected candidate sex and colour pattern genes used in this study. Each pathway involved at least one sex determination and one colour pattern genes, which are annotated in the same pathway.

\begin{tabular}{|c|c|c|c|}
\hline Pathway ID & Pathway name & Sex determination genes & Colour pattern genes \\
\hline 04020 & $\begin{array}{l}\text { Calcium signaling } \\
\text { pathway }\end{array}$ & lhcgr, pdgfra & $\begin{array}{l}\text { ednrba, egfra, erbb3a, } \\
\text { erbb3b, gnal la, gnallb, } \\
\text { gnaq }\end{array}$ \\
\hline 04110 & Cell cycle & $\operatorname{tp} 53$ & hdacl, mycb, zbtb17, rbl \\
\hline 00270 & $\begin{array}{l}\text { Cysteine and } \\
\text { methionine metabolism }\end{array}$ & $d n m t 3 b b .1, d n m t 3 b b .2$ & srm \\
\hline 04060 & $\begin{array}{l}\text { Cytokine-cytokine } \\
\text { receptor interaction }\end{array}$ & $a m h$ & $\begin{array}{l}\text { ghra, bmprlab, csflra, } \\
\text { csflrb, fas, eda }\end{array}$ \\
\hline 04144 & Endocytosis & prkcz, pdgfra & fgfr2, egfra, traf6 \\
\hline 04010 & $\begin{array}{l}\text { MAPK signaling } \\
\text { pathway }\end{array}$ & tp53, pdgfra & $\begin{array}{l}\text { egfra, casp } 3 a, n f l a, \text { fgfr } 2 \\
\text { mycb, map } 2 k 1, \text { traf6, nflb, } \\
\text { casp } 3 b, \text { fas }\end{array}$ \\
\hline 04916 & Melanogenesis & $w n t 4 a, c t n n b 1$, lef 1, wnt11 & $\begin{array}{l}\text { tyrpla, mitfa, mitfb, wntl, } \\
\text { tyr, kita, ednrba, lefl, mclr, } \\
\text { pomca, map } 2 k 1, \text { tyrplb, kitb, } \\
\text { kitlga, kitlgb, gnalla, gnaq, } \\
\text { crebla, dct, asipl }\end{array}$ \\
\hline 04137 & Mitophagy - animal & tp53 & mitfa, mitfb \\
\hline 03015 & $\begin{array}{l}\text { mRNA surveillance } \\
\text { pathway }\end{array}$ & $\operatorname{rbm} 8 a$ & pabpcla, pabpclb \\
\hline 04080 & $\begin{array}{l}\text { Neuroactive ligand- } \\
\text { receptor interaction }\end{array}$ & fshr, lhcgr, nr3cl & $\begin{array}{l}\text { ghra, s1pr2, drd2a, ednrba, } \\
m c 1 r, d r d 2 b\end{array}$ \\
\hline 03018 & RNA degradation & $\operatorname{exosc} 8$ & pabpcla, pabpclb, skiv2l2 \\
\hline 03013 & RNA transport & eefla1l1, rbm8a & pabpcla, pabpclb \\
\hline 04120 & $\begin{array}{l}\text { Ubiquitin mediated } \\
\text { proteolysis }\end{array}$ & fancl & mgrnla, mgrnlb, traf6 \\
\hline 03010 & Ribosome & rpl13 & rps19, rps20, rpl24 \\
\hline 03022 & $\begin{array}{l}\text { Basal transcription } \\
\text { factors }\end{array}$ & $t b p$ & $\operatorname{ercc} 2$ \\
\hline 04115 & p53 signaling pathway & tp53 & fas, casp $3 a$, casp $3 b$ \\
\hline 04140 & Autophagy - animal & tscla & $\begin{array}{l}\text { bcl2b, pdpklb, map } 2 k 1 \text {, } \\
\text { traf6, bcl2a }\end{array}$ \\
\hline 04145 & Phagosome & tuba4l, tuba7l & $\begin{array}{l}\text { itgblb.2,itgbla, } \\
\text { atp6apla,atp6v1elb, } \\
\text { atp6v0ca,atp6aplb, } \\
\text { atp6v1h,atp6v0d1, atp6v1f }\end{array}$ \\
\hline 04150 & $\begin{array}{l}\text { mTOR signaling } \\
\text { pathway }\end{array}$ & wnt $4 a$, tscla, wntll & $\begin{array}{l}\text { atp6vlelb,atp6v1h, wntl, } \\
\text { pdpk1b, map2k1, atp6vlf }\end{array}$ \\
\hline 04210 & Apoptosis & tp53, tuba4l, tuba7l & $\begin{array}{l}\text { bcl2b, casp } 3 a, \text { casp } 3 b \\
\text { pdpklb, map } 2 k 1, \text { bcl } 2 a, \text { fas }\end{array}$ \\
\hline 04310 & Wnt signaling pathway & $\begin{array}{l}\text { wnt } 4 a, \text { ctnnb1, tp53, } \\
\text { lef1, axin1, ctnnbip1, wnt11 }\end{array}$ & $w n t 1, a p c$, mycb, lef1 \\
\hline
\end{tabular}




\begin{tabular}{|c|c|c|c|}
\hline 04350 & $\begin{array}{l}\text { TGF-beta signaling } \\
\text { pathway }\end{array}$ & $a m h$ & mycb, bmprlab \\
\hline 04510 & Focal adhesion & ctnnb1, pdgfra & $\begin{array}{l}\text { bcl2b, egfra, pdpklb, } \\
\text { itgblb.2, map } 2 k 1, \text { itgbla, } \\
\text { pdgfc, bcl2a }\end{array}$ \\
\hline 04520 & Adherens junction & ctnnb1, lef1 & egfra, snai2, tjplb \\
\hline 04530 & Tight junction & gata4, tuba4l, tuba7l, prkcz & itgblb.2, itgbla, tjplb \\
\hline 04540 & Gap junction & tuba4l, tuba7l, pdgfra & $\begin{array}{l}d r d 2 a, \text { egfra, drd } 2 b, \\
\text { map2k1, gnal } 1 b, \text { tjplb, } \\
\text { pdgfc, gnalla, gnaq }\end{array}$ \\
\hline 04810 & $\begin{array}{l}\text { Regulation of actin } \\
\text { cytoskeleton }\end{array}$ & pdgfra & $\begin{array}{l}\text { fgfr2, egfra, apc, itgblb.2, } \\
\text { map2k1, itgbla, pdgfc }\end{array}$ \\
\hline 04910 & $\begin{array}{l}\text { Insulin signaling } \\
\text { pathway }\end{array}$ & tscla, prkcz & $p d p k 1 b$, map $2 k 1$ \\
\hline 04933 & $\begin{array}{l}\text { AGE-RAGE signaling } \\
\text { pathway in diabetic } \\
\text { complications }\end{array}$ & prkcz & bcl2b, casp $3 a$, casp $3 b, b c l 2 a$ \\
\hline 05168 & $\begin{array}{l}\text { Herpes simplex } \\
\text { infection }\end{array}$ & $t p 53, t b p$ & casp3a, traf6, casp3b, fas \\
\hline
\end{tabular}

\section{Protein-protein interaction networks}

To further inspect the interaction between annotated sex determination and colour pattern genes in the pathways, we used the STRING database (Szklarczyk et al., 2017), providing a biologically interconnected network in which nodes represent proteins and edges illustrate the variety of protein-protein interactions. Each node shows all proteins produced by a single protein-coding gene locus and these proteins jointly contribute to a shared function through an edge. The STRING analysis emphasizes the complexity of the association between sex and colour genes in form of a highly interconnected network (Figure 1). Among 94 annotated genes in pathway analysis (Table 1) as input parameter, we found 93 genes interacting with each other, resulting in 365 protein-protein associations based on the STRING database, in which these proteins jointly contributed to a shared function. The STRING output clearly showed that our set of annotated target genes in enriched KEGG pathways was biologically connected due to more intra-set interactions than would have been expected for a random set of proteins of similar size. This is true for the networks of our studied genes based on STRING result (protein-protein interaction enrichment $\mathrm{p}$-value $=1 \times 10^{-16}$ ). Within this large network, we identified the key regulator of sex and colour pattern genes such as tp53, lefl, $w n t 4 a$, ctnnbl, kita, mitfa, mycb and csflra that are highly interacted with each other as well as interact with other genes. 


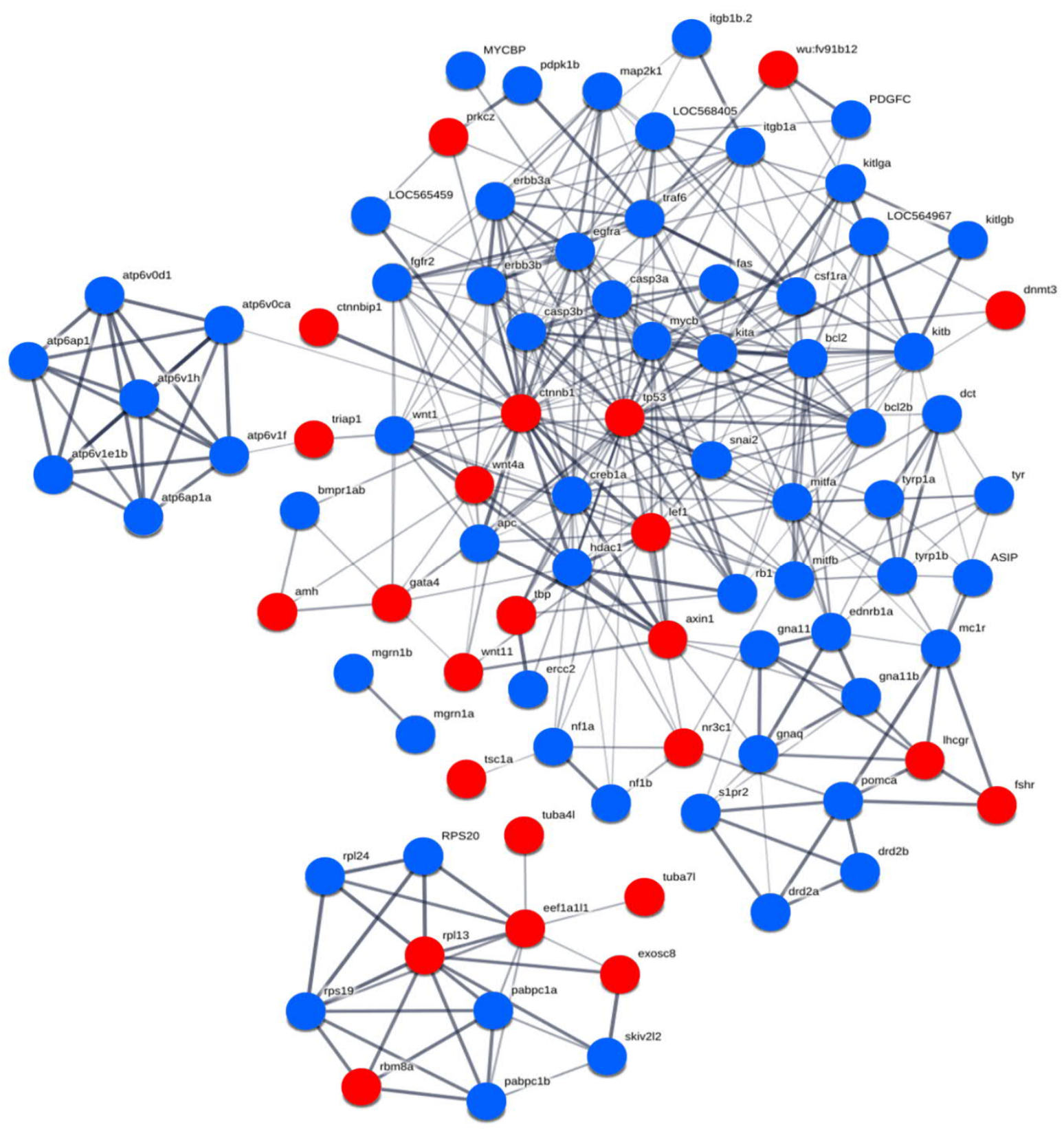

Figure 1: The result of interaction network map of annotated candidate sex determination and colour pattern genes in KEGG pathways using the STRING database. This graph only includes genes with at least one interaction with another gene. Nodes are proteins encoded by candidate genes and the edges represent protein-protein associations. The thickness of the edges indicates the strength of data (refers to the data in the STRING database) support for this association. Red nodes illustrate the sex determination genes, while the blue nodes illustrate the colour pattern genes. 


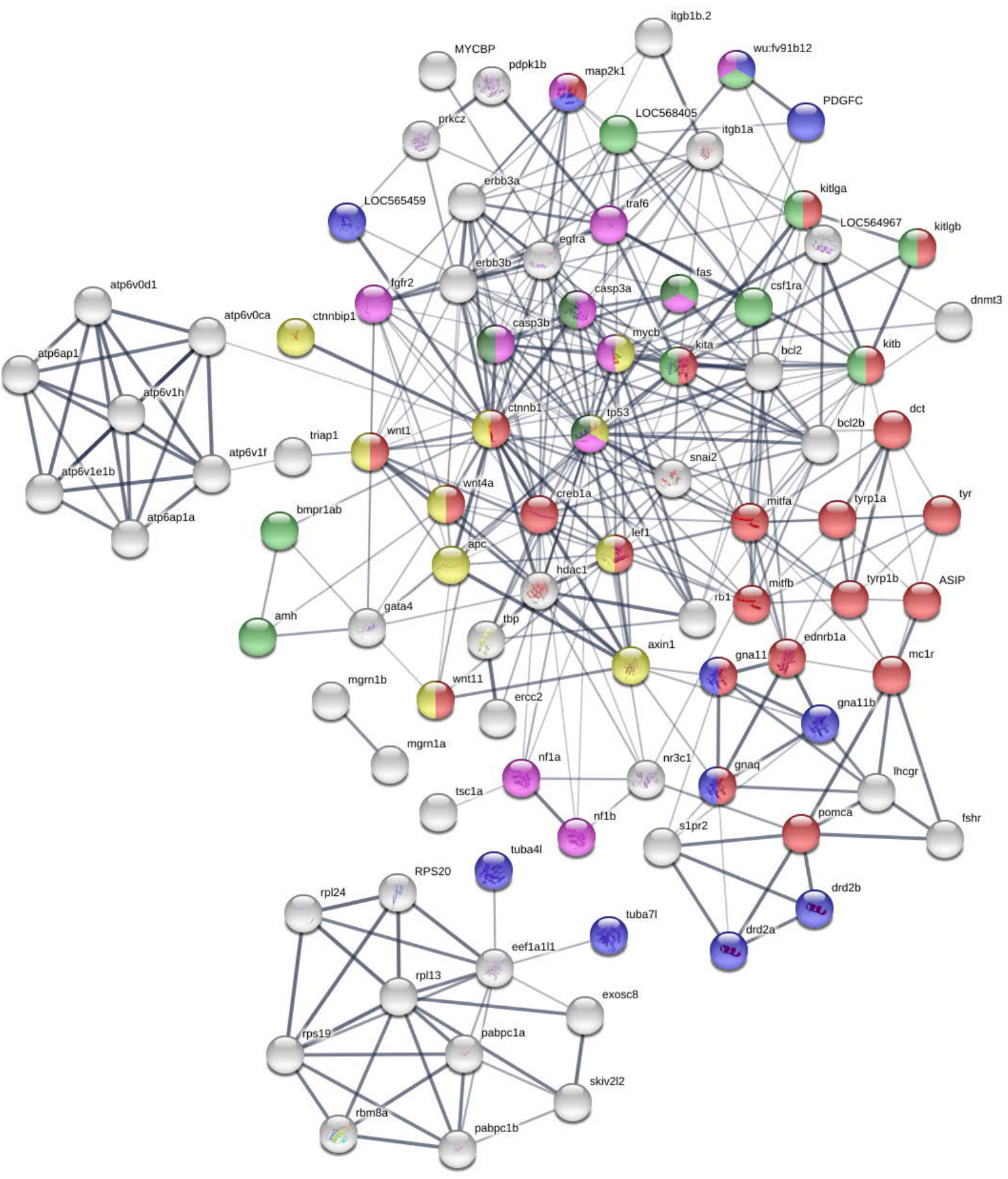

Figure 2: The result of interaction network between annotated target sex determination and colour pattern genes, which are annotated in different significant pathways according to the STRING database result. The annotated genes in different pathways are represented by different colour nodes including: red colour nodes illustrate Melanogenesis pathway, violet colour nodes illustrate Gap junction pathway, light green colour nodes illustrate Cytokine-cytokine receptor interaction pathway, yellow colour nodes illustrate Wnt signaling pathway, pink colour nodes illustrate MAPK signaling pathway and dark green colour nodes illustrate p53 signaling pathway. 
These genes with the highest rate of interconnections are located in the center of the network, suggesting the strong association between their proteins, as their coding genes were observed in the biological regulator pathways. According to the STRING output, a series of pathways was significantly enriched including the pathways with important roles in sex determination and colour pattern regulation as discussed above, such as Melanogenesis $\left(\mathrm{p}=2.79 \times 10^{-31}\right)$, Gap junction $\left(\mathrm{p}=2.94 \times 10^{-11}\right)$, Cytokine-cytokine receptor interaction $\left(\mathrm{p}=2.71 \times 10^{-10}\right)$, Wnt signaling pathway $\left(\mathrm{p}=9.98 \times 10^{-9}\right)$, MAPK signaling pathway $\left(\mathrm{p}=2.79 \times 10^{-7}\right)$ and $\mathrm{p} 53$ signaling pathway $(\mathrm{p}=0.00116)$. The interaction network between annotated genes in these pathways is illustrated in Figure 2.

\section{Specific and shared regulatory mechanisms of sex and colour genes}

The transcriptional regulation of gene expression in eukaryotes is essential for different cellular and biological processes. These processes in higher organisms are regulated by transcription factors and their combinatorial interplay, which are essential for complex genetic programs and the regulation of the transcriptional machinery in cells and in tissue development (Zeidler et al., 2016). Transcription factors are proteins, which act as activators or as repressors for the expression of genes by recruitment of RNA polymerase to ensure that the genes are expressed in the right cell type at the right time and in the appropriate level during cell differentiation and development of an organism throughout its life (Lee and Young, 2000; Jim, 2008). To regulate the transcription of the genes in the living organism, the transcription factors bind to the enhancer or promoter regions of DNA adjacent to the genes and regulate the expression of the genes to being either up- or down-regulated (Gill, 2001). The eukaryotic transcription machinery consists of two regulatory elements: 1) the cisacting elements, which are DNA sequences of the genome, and 2) the trans-acting elements, which are the transcription factors that identify and bind to the specific sequences in the cisacting elements at the transcription factor binding start sites to regulate the transcription of the target genes (Jim, 2008). The cis-acting regulatory elements in eukaryotes comprise two elements: promoters/proximal elements and distal regulatory regions. A promotor includes: 1) the core promotor, which usually consists of a TATA box (TATA), an initiator element (INR), a downstream promoter element (DPE), a motif ten element (MTE) and a TFIIB recognition element (BRE), and 2) the proximal promoter elements, which refer to the sequences upstream of the core promoter such as $\mathrm{CpG}$ islands. Distal regulatory regions comprise the enhancers, silencers or repressors, insulators and locus control regions, in which 
they can act in a coordinated fashion to regulate the expression of a gene (Figure 3) (Butler and Kadonaga, 2002; Jim, 2008; Wittkopp and Kalay, 2011). In order to initiate the transcription of the genes, the transcription factors must bind to the promoter region of DNA sequence once this region has been bound with the appropriate transcription factor or with a set of transcription factors in the proper order, in which the RNA polymerase can bind and start the transcription of a gene (Butler and Kadonaga, 2002).

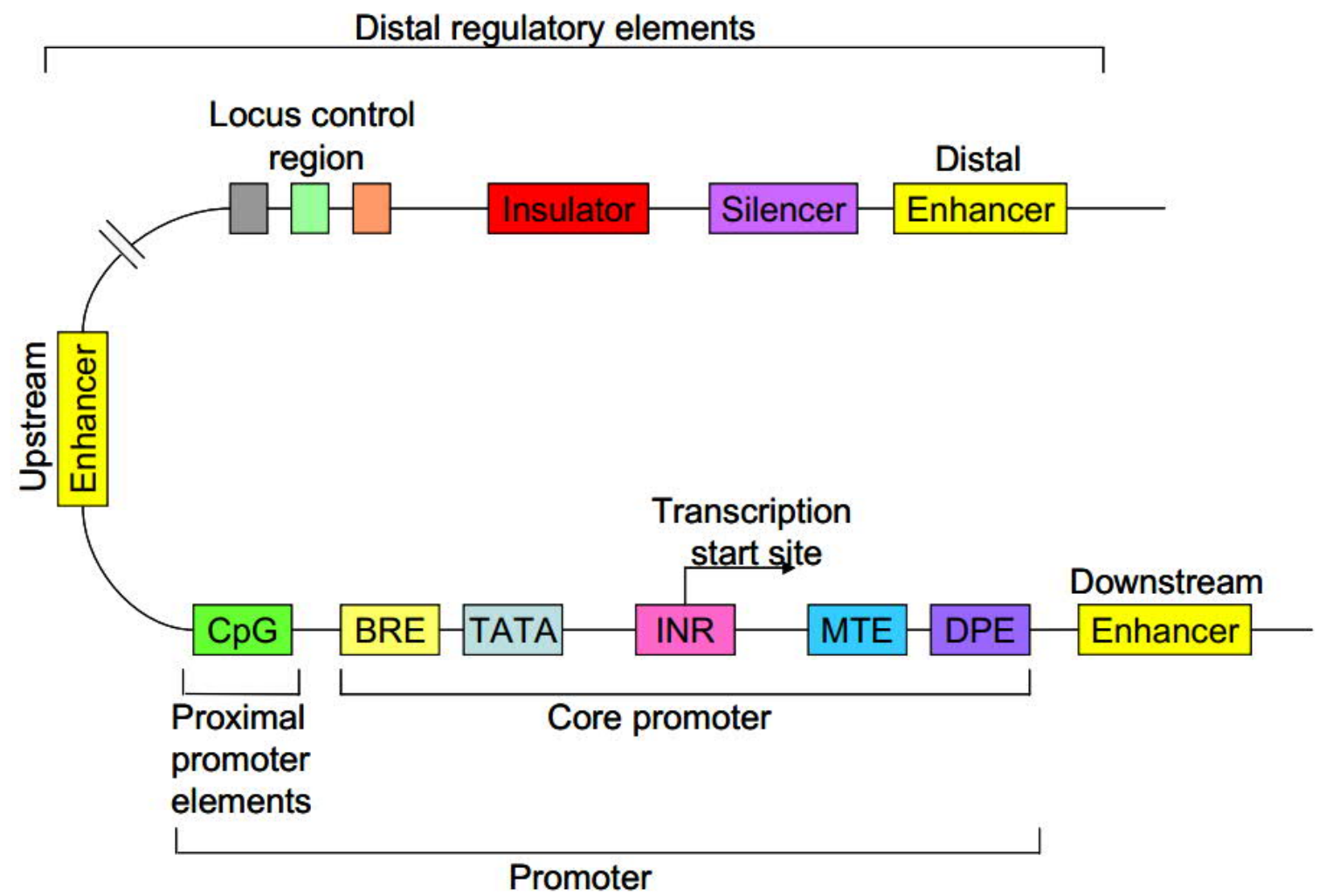

Figure 3: A schematic diagram of the cis-regulatory elements in eukaryotes. The core promoter contains a TATA box (TATA), an initiator element (INR), a downstream promoter element (DPE), a motif ten element (MTE) and a TFIIB recognition element (BRE). The proximal promoter elements such as $\mathrm{CpG}$ islands spanning about $1 \mathrm{~kb}$ around the transcription start site. The distal regulatory elements include enhancers, silencers, insulators and locus control regions (From Jim, 2008).

The role of specific transcription factor interactions in gene regulatory mechanisms has not been studied for sex determination and sex-associated colour pattern genes in zebrafish with respect to the phenotypic sexual dimorphism. In order to address this limited knowledge and to elucidate the association between sex and colour genes, we analyzed the selected candidate sex determination and colour pattern genes of zebrafish, which were used in chapter 4 of this 
thesis, to study the transcription regulation of sex and colour genes in order to find the transcription interactions between these two gene groups. For this purpose, we used a systematic approach to detect specific and common regulatory mechanisms between selected candidate sex and colour pattern genes, which combines identification of: i) sex and colour genes set-specific transcription factor interactions by analyzing the promoter regions (bioinformatics tool: PC-TraFF; Meckbach et al., 2015); ii) overrepresented pathways and master gene regulators to investigate the upstream processes using geneXplain platform (http://genexplain.com/genexplain-platform/); and iii) comparison of the results of both sex and colour genes for their shared regulatory mechanisms.

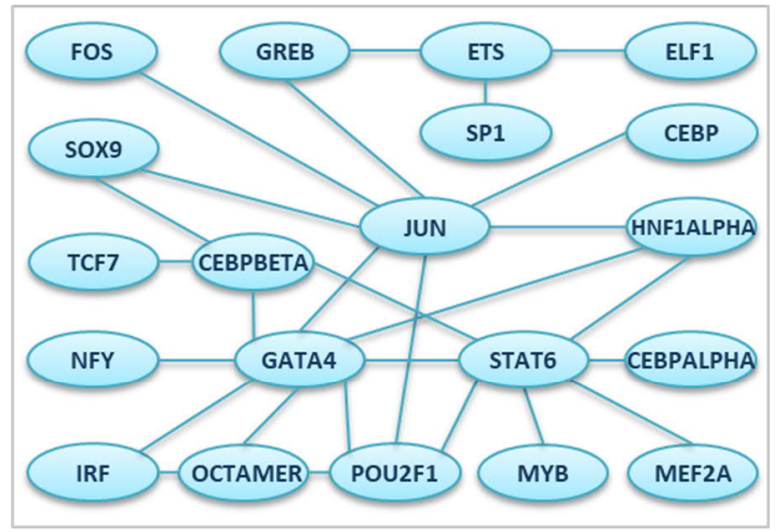

Specific TF interactions for sex determination genes

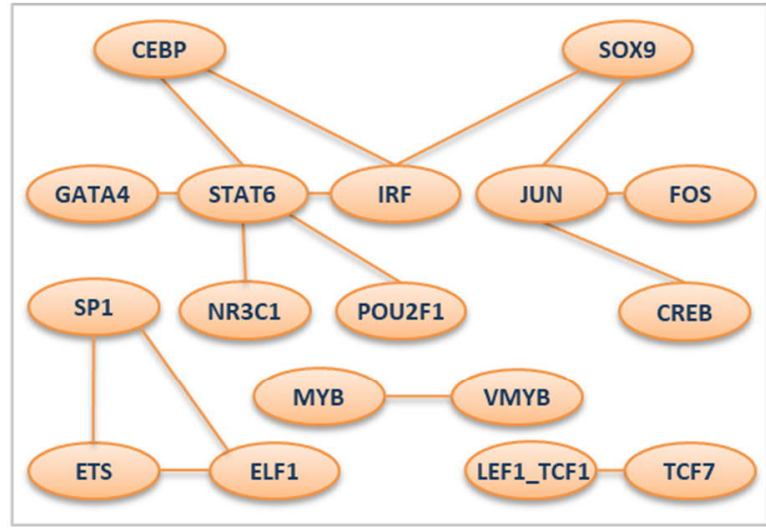

Specific TFs interactions for colour pattern genes

Common TF interactions for sex and colour genes

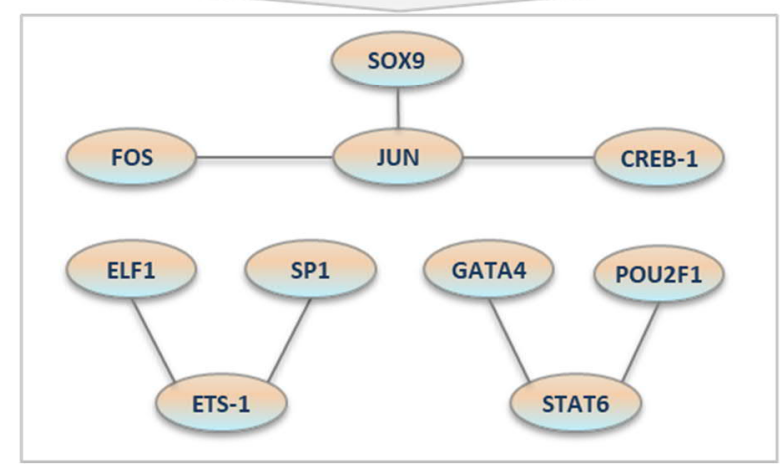

Figure 4: The result of identified specific and common regulatory mechanisms (transcription factor interactions) of selected candidate sex determination and colour pattern genes, which were used in this thesis. TF: Transcription Factor

We found 27 and 15 transcription factor interactions to be significant for sex determination and colour pattern, respectively (Figure 4). From these, seven interactions have been identified as shared regulatory elements for both gene groups, in which SOX9, GATA4, SP1 
and STAT6 are known to play an important role for sex determination and colour patterning. Furthermore, the investigation of upstream processes provides pivotal knowledge about a shared master regulator (PKACA) for both sex and colour genes. Protein kinase A Catalytic Subunit A (PKACA), which encodes by PRKACA gene, is a key regulatory enzyme in phosphorylating other proteins and substrates, and changing their activity (Taskén et al., 1996). PRKACA gene is a part of a different pathway involved in colour pattern and sex determination such as Wnt signaling pathway, gap junction, tight junction, MAPK signaling pathway and melanogenesis, as identified in KEGG database. Since we found the common regulatory mechanisms and a shared master regulator for sex and colour genes, these provide evidence for the association between sex determination and colour pattern genes of zebrafish. The results of the transcription factor analysis are being further investigated and a paper is being prepared in the next step of our study.

\section{Outlook for future research}

Sex determination in zebrafish is a result of complex combination between genetics and environmental interactions. High ambient temperature leads to an increase in proportion of males and consequently an unbalanced in sex ratio in zebrafish as a model animal. This temperature effect causes a reduction in reproductive capacity at the population level and changes the composition and function of ecosystems in nature. Despite numerous efforts, our understanding of the mechanism of sex determination in this species is not still complete. Many important open questions remain to be answered: How temperature can induce masculinization during thermosensitive embryonic and larval developmental periods, and how it can influence and modify the genetic mechanism to alter the sex. Environmental effects can induce epigenetic modifications of chromatin, which in turn influence the regulation of gene expression resulted in phenotypic variations. Further epigenome study on chromatin modification, including DNA methylation or histone modifications, may elucidate the role of temperature in regulating the mechanism of sex determination in zebrafish. Besides to the effect of temperature on masculinization, this thesis reported its influence on the intensity of colour pattern in zebrafish. This appeared curious and led to a question that had never been explored before: Is there an epigenetic reason for colour intensity modifications? Since chromatophores arise from the neural crest during embryonic development, but the colour pattern in adult zebrafish is developed during metamorphosis, a longitudinal study of the effect of temperature on colouration during zebrafish development is 
proposed. In addition, taking into account the fact that there is a genetic difference in sex determination mechanism between domesticated and wild populations: Is there a genomic structural variation in different populations? Does the temperature interact differently in both natural and laboratory strains? Studies we plan for the future will answer the questions arising from this thesis in order to gain more knowledge of the complex genetic mechanisms underlying phenotypic plasticity in interaction with high ambient temperature in zebrafish and related species.

\section{Main conclusions from this thesis}

In this thesis, the genetic and phenotypic plasticity of sex determination in interaction with temperature in zebrafish was studied. The main conclusions of this thesis can be summarized as follows:

1) Increased water temperature during embryogenesis in zebrafish induced masculinization and changed the sex ratio. This result emphasizes the interplay between genetic and environmental factors and confirms the polygenic sex determination system in laboratory strains of zebrafish.

2) In this study, a reduction of survival ability was recorded during embryonic and postembryonic development in response to high water temperature. In contrast, a positive effect of elevated temperature on growth performance was observed in temperature-induced animals with a greater impact in females, suggesting that high temperature may increase the expression of growth-related genes.

3) In this thesis, fully automatic sex determination using machine learning methods (DCNNs and SVM) was performed for the first time in zebrafish, which have the advantage of automation and robustness with a high degree of accuracy compared to conventional methods.

4) Using SVM, we have shown that males exhibit intense colour in the caudal fin compared to females, which has not been reported in zebrafish so far. A reduction of colour intensity was detected in the caudal fin in some heat-exposed males, suggesting that these animals are likely to be masculinized or neomales.

5) A positive association of caudal fin colouration with body size was observed in males, which may play a role in sexual selection. 
6) Transcriptome analysis of gonad showed the activation of pro-male gene expression and repression of pro-female gene expression in male gonads compared to females, leading to gonadal masculinization.

7) No significant impact of the temperature treatment or the sex was observed in the expression level of the colour pattern genes; although a high expression level of those genes was observed in the caudal fin of adult zebrafish in both sexes. A significantly differentially expression of colour pattern genes in the gonad of both sexes was identified, suggesting the neofunctionalisation of those genes in the zebrafish reproduction system.

8) A subset of pathways containing sex determination and colour pattern genes were enriched in this study, which provide the evidence for the association of sex and colour genes in zebrafish regarding the phenotypic sexual dimorphism in adult animals.

\section{References}

Abozaid H, Wessels S, Hörstgen-Schwark G. Effect of rearing temperatures during embryonic development on the phenotypic sex in zebrafish (Danio rerio). Sex Dev. $2011 ; 5: 259-265$.

Angermueller C, Pärnamaa T, Parts L, Stegle O. Deep learning for computational biology. Mol Syst Biol. 2016; 12:878.

Baroiller JF, D'Cotta H, Saillant E. Environmental effects on fish sex determination and differentiation. Sex Dev. 2009; 3:118-35.

Braasch I, Brunet F, Volff JN, Schartl M. Pigmentation pathway evolution after WholeGenome duplication in Fish. Genome Biol Evol. 2009; 1:479-493.

Brown AR, Owen SF, Peters J, Zhang Y, Soffker M, Paull GC, Hosken DJ, Wahab MA, Tyler CR. Climate change and pollution speed declines in zebrafish populations. Proc Natl Acad Sci U S A. 2015; 112:E1237-1246.

Butler JE, Kadonaga JT. The RNA polymerase II core promoter: a key component in the regulation of gene expression. Genes Dev. 2002; 16: 2583-92.

Ceinos RM, Guillot R, Kelsh RN, Cerdá-Reverter JM, Rotllant J. Pigment patterns in adult fish result from superimposition of two largely independent pigmentation mechanisms. Pigment Cell Melanoma Res. 2015; 28:196-209. 
Cheung WWL, Sarmiento JL, Dunne J, Frölicher TL, Lam VWY, Palomares D, Watson R, Pauly D. Shrinking of fishes exacerbates impacts of global ocean changes on marine ecosystems. Nat Clim Change. 2013; 3:254-258.

Díaz N, Piferrer F. Lasting effects of early exposure to temperature on the gonadal transcriptome at the time of sex differentiation in the European sea bass, a fish with mixed genetic and environmental sex determination. BMC Genomics. 2015; 16:679.

Dooley CM, Mongera A, Walderich B, Nüsslein-Volhard C. On the embryonic origin of adult melanophores: the role of ErbB and Kit signalling in establishing melanophore stem cells in zebrafish. Development. 2013;140:1003-13.

Fadeev A, Krauss J, Frohnhöfer HG, Irion U, Nüsslein-Volhard C, et al. Tight Junction Protein 1a regulates pigment cell organisation during zebrafish colour patterning. Elife. 2015; 4:e06545.

Feary DA, Pratchett MS, Emslie MJ et al. Latitudinal shifts in coral reef fishes: why some species do and others do not shift. Fish Fish. 2014; 15:593-615.

Frohnhöfer HG, Krauss J, Maischein HM, Nüsslein-Volhard C. Iridophores and their interactions with other chromatophores are required for stripe formation in zebrafish. Development. 2013;140:2997-3007.

Gill G. Regulation of the initiation of eukaryotic transcription. Essays Biochem. 2001; 37: 33-43.

Guyader LS, Maier J, Jesuthasan S. Esrom, an ortholog of PAM (protein associated with cmyc), regulates pteridine synthesis in the zebrafish. Dev Biol. 2005; 277:378-86.

Habary A, Johansen JL, Nay TJ, Steffensen JF, Rummer JL. Adapt, move or die - how will tropical coral reef fishes cope with ocean warming?. Glob Chang Biol. 2017; 23:566577.

Huey RB, Kingsolver JG. Evolution of resistance to high temperature in ectotherms. Am Nat. $1993 ; 142: 21-46$.

Hultman KA, Bahary N, Zon LI, Johnson SL. Gene duplication of the zebrafish kit ligand and partitioning of melanocyte development functions to kit ligand a. PLoS Genet. 2007; 3:e17.

IPCC Climate Change 2013: The Physical Science Basis. Working Group I Contribution to the Fift Assessment Report of the Intergovernmental Panel on Climate Change. 2013; Cambridge University Press, Cambridge, UK.

Irion U, Frohnhöfer HG, Krauss J, Champollion TC, Maischein HM, Geiger-Rudolph S, Weiler C, Nüsslein-Volhard C, Bronner ME. Gap junctions composed of connexions 
41.8 and 39.4 are essential for color pattern formation in zebrafish. Elife. 2014; 3:e05125.

Jim HL. Identification of Target Genes of an Erythroid Transcription Factor Complex Containing SCL (TAL1). Dissertation, University of Cambridge. 2008; pp.1-7.

Johnson SL, Nguyen AN, Lister JA. mitfa is required at multiple stages of melanocyte differentiation but not to establish the melanocyte stem cell. Dev Biol. 2011; 350:40513.

Jyväsjärvi J, Marttila H, Rossi PM, Ala-Aho P, Olofsson B, Nisell J, Backman B, Ilmonen J, Virtanen R, Paasivirta L, Britschgi R, Kløve B, Muotka T. Climate-induced warming imposes a threat to north European spring ecosystems. Glob Chang Biol. 2015; 21:4561-9.

Kanehisa M, Goto S, Sato Y, Furumichi M, Tanabe M. KEGG for integration and interpretation of large-scale molecular data sets. Nucleic Acids Res. 2012; 40:D109-14.

LeCun Y, Bengio Y, Hinton G. Deep learning. Nature. 2015; 521:436-44.

Lee SLJ, Horsfield JA, Black MA, Rutherford K, Fisher A, Gemmell NJ. Histological and transcriptomic effects of $17 \alpha$-methyltestosterone on zebrafish gonad development. BMC Genomics. 2017;18:557.

Lee TI, Young RA. Transcription of eukaryotic protein-coding genes. Annu Rev Genet. 2000; 34: 77-137.

Liakos KG, Busato P, Moshou D, Pearson S, Bochtis D. Machine Learning in Agriculture: A Review. Sensors (Basel). 2018; 18:E2674.

Liew WC, Orbán L. Zebrafish sex: A complicated affair. Brief. Funct. Genomics. 2014; 13:172-187.

Liu W, Li SZ, Li Z, Wang Y, Li XY, Zhong JX, Zhang XJ, Zhang J, Zhou L, Gui JF. Complete depletion of primordial germ cells in an All-female fish leads to Sex-biased gene expression alteration and sterile All-male occurrence. BMC Genomics. 2015; 16:971.

Lough JM. Small change, big difference: sea surface temperature distributions for tropical coral reef ecosystems, 1950-2011. J Geophys Res. 2012; 117:C09018.

McMillan SC, Géraudie J, Akimenko MA. Pectoral fin breeding tubercle clusters: a method to determine zebrafish sex. Zebrafish. 2015; 12:121-3. 
Messmer V, Pratchett MS, Hoey AS, Tobin AJ, Coker DJ, Cooke SJ, Clark TD. Global warming may disproportionately affect larger adults in a predatory coral reef fish. Glob Chang Biol. 2017; 23:2230-2240.

Nüsslein-Volhard C, Singh AP. How fish colour their skin: A paradigm for development and evolution of adult patterns. Bioessays. 2017; 39:3.

Ohlberger J. Climate warming and ectotherm body size - from individual physiology to community ecology. Funct Ecol. 2013; 27:991-1001.

Ospina-Alvarez N, Piferrer F. Temperature-dependent sex determination in fish revisited: prevalence, a single sex ratio response pattern, and possible effects of climate change. PLoS One. 2008; 3:e2837.

Pandit SN, Maitland BM, Pandit LK, Poesch MS, Enders EC. Climate change risks, extinction debt, and conservation implications for a threatened freshwater fish: Carmine shiner (Notropis percobromus). Sci Total Environ. 2017; 598:1-11.

Parichy DM1, Turner JM. Temporal and cellular requirements for Fms signaling during zebrafish adult pigment pattern development. Development. 2003; 130:817-33.

Patterson LB, Bain EJ, Parichy DM. Pigment cell interactions and differential xanthophore recruitment underlying zebrafish stripe reiteration and Danio pattern evolution. Nat Commun. 2014; 5:5299.

Patterson LB, Parichy DM. Interactions with iridophores and the tissue environment required for patterning melanophores and xanthophores during zebrafish adult pigment stripe formation. PLoS Genet. 2013;9:e1003561.

Piferrer F, Ribas L, Díaz N. Genomic approaches to study genetic and environmental influences on fish sex determination and differentiation. Mar Biotechnol. 2012; 14:591-604.

Poff NL, Brinson MM, Day JW. Aquatic Ecosystems \& Global Climate Change - Potential impacts on inland freshwater and coastal wetland ecosystems in the United States. Prepared for the Pew Center on Global Climate Change, 2002, pp.1-45.

Poonlaphdecha S, Pepey E, Canonne M, de Verdal H, Baroiller JF, D'Cotta H. Temperature induced-masculinisation in the Nile tilapia causes rapid up-regulation of both dmrt 1 and amh expressions. Gen Comp Endocrinol. 2013; 193:234-242.

Pyron, M. Female preferences and male-male interactions in zebrafish (Danio rerio). Can J Zool. 2003; 81:122-125.

$\mathrm{R}$ Core Team. R: a language and environment for statistical computing. Vienna: $\mathrm{R}$ Foundation for Statistical Computing; 2015. URL https://www.Rproject.org/. 
Ribas L, Liew WC, Díaz N, Sreenivasan R, Orbán L, Piferrer F. Heat-induced masculinization in domesticated zebrafish is family-specific and yields a set of different gonadal transcriptomes. Proc Natl Acad Sci U S A. 2017; 114:E941-E950.

Rodríguez-Marí A, Cañestro C, BreMiller RA, Nguyen-Johnson A, Asakawa K, Kawakami $\mathrm{K}$, Postlethwait JH. Sex reversal in zebrafish fancl mutants is caused by Tp53-mediated germ cell apoptosis. PLoS Genet. 2010; 6:e1001034.

Serrana DGDI, Vieira VL, Andree KB, Darias M, Estévez A, Gisbert E, Johnston IA. Development temperature has persistent effects on muscle growth responses in gilthead sea bream. PLoS One. 2012; 7:e51884.

Sharma E, Künstner A, Fraser BA, Zipprich G, Kottler VA, Henz SR, Weigel D, Dreyer C. Transcriptome assemblies for studying sex-biased gene expression in the guppy, Poecilia reticulate. BMC Genomics. 2014;15:400.

Sharma S, Jackson DA, Minns CK, Shuter BJ. Will northern fish populations be in hot water because of climate change?. Glob Change Biol. 2007; 13:2052-2064.

Shen ZG, Wang HP. Molecular players involved in temperature-dependent sex determination and sex differentiation in Teleost fish. Genet Sel Evol. 2014; 46:26.

Singh AP, Frohnhöfer HG, Irion U, Nüsslein-Volhard C. Response to comment on "Local reorganization of xanthophores fine-tunes and colours the striped pattern of zebrafish. Science. 2015; 348:297.

Singh AP, Nüsslein-Volhard C. Zebrafish Stripes as a Model for Vertebrate Colour Pattern Formation. Curr Biol. 2015; 25: R81-R92.

Skinner AMJ, Watt PJ. Strategic egg allocation in the zebra fish, Danio rerio. Behav Ecol. 2007; 18:905-909.

Solomon S, Qin D, Manning M, Chen Z, Marquis M, Averyt K, Tignor M, Miller H: IPCC, Climate Change 2007: The Physical Science Basis. Cambridge: Cambridge University Press; 2007.

Sreenivasan R, Jiang J, Wang X, Bartfai R, Kwan HY, Christoffels A, Orban L. Gonad differentiation in zebrafish is regulated by the canonical Wnt signaling pathway. Biol Reprod. 2014; 90:45-56.

Szklarczyk D, Morris JH, Cook H, Kuhn M, Wyder S, Simonovic M, Santos A, Doncheva NT, Roth A, Bork P, Jensen LJ, von Mering C. The STRING database in 2017: qualitycontrolled protein-protein association networks, made broadly accessible. Nucleic Acids Res. 2017; 45:D362-D368. 
Taskén K, Solberg R, Zhao Y, Hansson V, Jahnsen T, Siciliano MJ. The gene encoding the catalytic subunit $\mathrm{C}$ alpha of cAMP-dependent protein kinase (locus PRKACA) localizes to human chromosome region 19p13.1. Genomics. 1996; 36: 535-8.

Tewksbury JJ, Huey RB, Deutsch CA. Putting the heat on tropical animals. Science, 2008; 320:1296.

Tzung KW, Goto R, Saju JM, Sreenivasan R, Saito T, Arai K, Yamaha E, Hossain MS, Calvert ME, Orbán L. Early depletion of primordial germ cells in zebrafish promotes testis formation. Stem Cell Reports. 2015; 4:61-73.

Uchida D, Yamashita M, Kitano T, Iguchi T: Oocyte apoptosis during the transition from ovary-like tissue to testes during sex differentiation of juvenile zebrafish. J Exp Biol. 2002; 205:711-718.

Uusi-Heikkilä, S., Böckenhoff, L., Wolter, C., \& Arlinghaus, R. Differential allocation by female zebrafish (Danio rerio) to different-sized males--an example in a fish species lacking parental care. PLoS One. 2012; 7:e48317.

Wang XG, Orban L. Anti-Müllerian hormone and 11 beta-hydroxylase show reciprocal expression to that of aromatase in the transforming gonad of zebrafish males. Dev Dyn. 2007; 236:1329-38.

Webster KA, Schach U, Ordaz A, Steinfeld JS, Draper BW, Siegfried KR. Dmrt1 is necessary for male sexual development in zebrafish. Dev Biol. 2017; 422:33-46.

Wittkopp PJ, Kalay G. Cis-regulatory elements: molecular mechanisms and evolutionary processes underlying divergence. Nat Rev Genet. 2011; 13:59-69.

Zeidler S, Meckbach C, Tacke R, Raad FS, Roa A, Uchida S, Zimmermann WH, Wingender E, Gültas M. Computational Detection of Stage-Specific Transcription Factor Clusters during Heart Development. Front Genet. 2016; 7:33. 
I would like to thank:

Prof. Dr. Henner Simianer. Thank you very much for your supervising and great support and constructive suggestions and discussions to carry out my doctoral project.

Prof. Dr. Klaus Wimmers. Thank you very much for the co-supervising of my $\mathrm{PhD}$ project and valuable discussions for further planned research work.

Prof. Dr. Dr. Bertram Brenig. Thank you very much for the useful conversations, discussions and suggestions during the completion of my doctoral thesis and welcoming me so kindly to work in your laboratory for a long time.

Dr. A. Reza Sharifi. I am very grateful for all your kind and helpful advices and support me during my $\mathrm{PhD}$ project. Thank you so much for encouraging me to overcome the difficulties during my doctoral thesis.

Dr. Marc Hirschfeld. I wish to express my gratitude to you for helping me to learn the molecular genetic techniques.

Prof. Dr. Jens Tetens. Thank you very much for the good discussions and provide the possibility to use your laboratory.

I am thankful to the Prof. Dr. Hörstgen-Schwark and Aquaculture and Aquatic Ecology staff to provide the possibility to use the aquaculture facilities for producing animals.

I extend my thanks to all my colleagues and friends at the University of Göttingen, specially Dr. Ngoc-Thuy Ha, Dr. Clemens Falker-Gieske and Sebastian Herzog for their contribution to this research work.

I am deeply grateful to my parents and my dear brother for their love, patient and understanding during all these years and their never-ending support. 